\title{
When binaries keep track of recent nucleosynthesis The $\mathrm{Zr}-\mathrm{Nb}$ pair in extrinsic stars as an s-process diagnostic
}

\author{
D. Karinkuzhi ${ }^{1}$, S. Van Eck ${ }^{1}$, A. Jorissen ${ }^{1}$, S. Goriely ${ }^{1}$, L. Siess ${ }^{1}$, T. Merle ${ }^{1}$, A. Escorza ${ }^{1,2}$, M. Van der Swaelmen ${ }^{1}$, \\ H. M. J. Boffin ${ }^{3}$, T. Masseron ${ }^{4,5}$, S. Shetye ${ }^{1,2}$, and B. Plez ${ }^{6}$ \\ ${ }^{1}$ Institut d'Astronomie et d'Astrophysique, Université Libre de Bruxelles, ULB, Campus Plaine C.P. 226, Boulevard du Triomphe, \\ 1050 Bruxelles, Belgium \\ e-mail: dkarinku@ulb.ac.be, svaneck@astro.ulb.ac.be \\ 2 Institute of Astronomy, KU Leuven, Celestijnenlaan 200D, 3001 Leuven, Belgium \\ 3 ESO, Karl Schwarzschild Straße 2, 85748 Garching bei München, Germany \\ 4 Instituto de Astrofísica de Canarias, 38205 La Laguna, Tenerife, Spain \\ 5 Departamento de Astrofísica, Universidad de La Laguna, 38206 La Laguna, Tenerife, Spain \\ ${ }^{6}$ Laboratoire Univers et Particules de Montpellier, Université Montpellier, CNRS, 34095 Montpellier Cedex 05, France
}

Received 23 March 2018 / Accepted 28 May 2018

\begin{abstract}
Context. Barium stars are s-process enriched giants. They owe their chemical peculiarities to a past mass transfer phase. During this phase they were polluted by their binary companion, which at the time was an asymptotic giant branch (AGB) star, but is now an extinct white dwarf. Barium stars are thus ideal targets for understanding and constraining the $s$-process in low- and intermediate-mass AGB stars.

Aims. We derive the abundances of a large number of heavy elements in order to shed light on the conditions of operation of the neutron source responsible for the production of $s$-elements in the former companions of the barium stars.

Methods. Adopting a recently used methodology, we analyse a sample of eighteen highly enriched barium stars observed with the high-resolution HERMES spectrograph mounted on the Mercator telescope (La Palma). We determine the stellar parameters and abundances using MARCS model atmospheres. In particular, we derive the $\mathrm{Nb}-\mathrm{Zr}$ ratio which was previously shown to be a sensitive thermometer for the $s$-process nucleosynthesis. Indeed, in barium stars, ${ }^{93} \mathrm{Zr}$ has fully decayed into mono-isotopic ${ }^{93} \mathrm{Nb}$, so $\mathrm{Nb} / \mathrm{Zr}$ is a measure of the temperature-sensitive ${ }^{93} \mathrm{Zr} / \mathrm{Zr}$ isotopic ratio.

Results. HD 28159, previously classified as K5III and initially selected to serve as a reference cool K star for our abundance analysis, turns out to be enriched in $s$-process elements, and as such is a new barium star. Four stars are characterised by high nitrogen abundances, and among those three have high $[\mathrm{Nb} / \mathrm{Zr}]$ and $[\mathrm{hs} / \mathrm{ls}]$ ratios. The derived $\mathrm{Zr}$ and $\mathrm{Nb}$ abundances provide more accurate constraints on the $s$-process neutron source, identified to be ${ }^{13} \mathrm{C}(\alpha, \mathrm{n}){ }^{16} \mathrm{O}$ for barium stars. The comparison with stellar evolution and nucleosynthesis models shows that the investigated barium stars were polluted by a low-mass $\left(M \sim 2-3 M_{\odot}\right)$ AGB star. HD 100503 is potentially identified as a high metallicity analogue of carbon-enhanced metal-poor star enriched in both $r$ - and $s$-process elements (CEMP-rs).
\end{abstract}

Key words. nuclear reactions, nucleosynthesis, abundances - stars: AGB and post-AGB - binaries: spectroscopic stars: fundamental parameters

\section{Introduction}

Low- and intermediate-mass asymptotic giant branch (AGB) stars are major contributors to the chemical evolution of the Galaxy, especially in the case of carbon and elements heavier than iron produced by the $s$-process (Käppeler 1999; Käppeler et al. 2011) and perhaps for nitrogen as well (e.g. Merle et al. 2016). There are still many open questions about the exact physical conditions required for the occurrence of these nucleosynthesis processes. On the AGB, the first stars to exhibit surface signatures of $s$-process nucleosynthesis are S-type stars, which have prominent $\mathrm{ZrO}$ bands and $s$-process overabundances. However, their low photospheric temperatures cause a strong molecular blending throughout their spectrum, making it very difficult to study their abundances in detail (Smith \& Lambert 1990; Van Eck et al. 2017). The problem is even more accute for carbon stars. In this respect, extrinsic stars can be an invaluable help.
The concept of extrinsic stars was first introduced by Iben \& Renzini (1983) to distinguish the S stars, which owe their $s$-process enrichment to mass transfer from an AGB companion, from the intrinsic $\mathrm{S}$ stars, which are genuine AGB stars whose atmospheres are enriched by $s$-process material produced in their interiors. Extrinsic S stars, which do not exhibit Tc lines (an element with no stable isotopes), populate the tip of the RGB or the early AGB; as expected, they are all binaries and do not show infrared excesses, unlike their more evolved intrinsic counterparts (Jorissen et al. 1993; Van Eck \& Jorissen 1999, 2000; Van Eck et al. 2000). The subclass of K giants known as barium stars (Bidelman \& Keenan 1951) are the warmer counterparts of extrinsic S stars (Smith \& Lambert 1988; Jorissen \& Mayor 1988), whereas CH giants (Keenan 1942) and carbon-enhanced metal-poor (CEMP) stars (at least those enriched with $s$-process elements, the so-called CEMP-s; Masseron et al. 2010) are the low-metallicity counterparts of the barium stars. A more detailed 
Table 1. Programme stars, and adopted atmospheric parameters ( $\xi$ is the microturbulence velocity).

\begin{tabular}{|c|c|c|c|c|c|c|c|}
\hline Name & $\begin{array}{l}T_{\text {eff }} \\
(\mathrm{K})\end{array}$ & $\begin{array}{c}\log g \\
\left(\mathrm{~cm} \mathrm{~s}^{-2}\right)\end{array}$ & $\begin{array}{c}\xi \\
\left(\mathrm{km} \mathrm{s}^{-1}\right)\end{array}$ & {$[\mathrm{Fe} / \mathrm{H}]$} & $\mathrm{S} / \mathrm{N}$ & $\begin{array}{l}\text { Spectral } \\
\text { type }\end{array}$ & Remark \\
\hline HD 5424 & $4728 \pm 80$ & $2.53 \pm 0.02$ & $1.30 \pm 0.05$ & $-0.43 \pm 0.11$ & 75 & G8II & BACCHUS \\
\hline HD 12392 & $4987 \pm 17$ & $3.39 \pm 0.30$ & $1.34 \pm 0.04$ & $-0.38 \pm 0.09$ & 105 & G8II & BACCHUS \\
\hline HD 16458 & $4550 \pm 25$ & $1.80 \pm 0.20$ & $1.92 \pm 0.05$ & $-0.64 \pm 0.10$ & 139 & $\mathrm{~K} 1 \mathrm{Ba} 5$ & $\chi^{2}$ method \\
\hline HD 27271 & $5022 \pm 40$ & $2.86 \pm 0.50$ & $1.29 \pm 0.03$ & $-0.07 \pm 0.08$ & 151 & G8II & BACCHUS \\
\hline HD 28159 & $3900 \pm 50$ & $2.00 \pm 0.50$ & 2.00 & $-0.50 \pm 0.13$ & 83 & K5III & $\chi^{2}$ method \\
\hline HD 31487 & $4960 \pm 50$ & $3.11 \pm 0.20$ & $1.45 \pm 0.03$ & $-0.04 \pm 0.13$ & 120 & G8IIIBa3 & BACCHUS \\
\hline HD 43389 & $4000 \pm 50$ & $2.00 \pm 0.50$ & 2.00 & $-0.35 \pm 0.13$ & 131 & K0/1IIICNIIp & $\chi^{2}$ method \\
\hline HD 46407 & $4854 \pm 100$ & $2.24 \pm 0.40$ & $1.34 \pm 0.05$ & $-0.36 \pm 0.11$ & 127 & G9III & BACCHUS \\
\hline HD 50082 & $4789 \pm 100$ & $2.44 \pm 0.50$ & $1.36 \pm 0.05$ & $-0.32 \pm 0.11$ & 86 & $\mathrm{~K} 0 \mathrm{Ba} 3$ & BACCHUS \\
\hline HD 60197 & $3800 \pm 50$ & $2.00 \pm 0.50$ & 2.00 & $-0.60 \pm 0.12$ & 105 & K3.5III & $\chi^{2}$ method \\
\hline HD 88562 & $4000 \pm 50$ & $2.00 \pm 0.50$ & 2.00 & $-0.53 \pm 0.12$ & 83 & K1III & $\chi^{2}$ method \\
\hline HD 100503 & $4000 \pm 50$ & $2.00 \pm 0.50$ & 2.00 & $-0.72 \pm 0.13$ & 79 & $\mathrm{G} / \mathrm{KpBa}$ & $\chi^{2}$ method \\
\hline HD 116869 & $4892 \pm 30$ & $2.59 \pm 0.07$ & $1.39 \pm 0.04$ & $-0.44 \pm 0.09$ & 64 & G8III & BACCHUS \\
\hline HD 120620 & $4831 \pm 13$ & $3.03 \pm 0.30$ & $1.11 \pm 0.05$ & $-0.30 \pm 0.10$ & 69 & K0III & BACCHUS \\
\hline HD 121447 & $4000 \pm 50$ & $1.00 \pm 0.50$ & 2.00 & $-0.90 \pm 0.13$ & 73 & K4III & $\chi^{2}$ method \\
\hline HD 123949 & $4378 \pm 80$ & $1.78 \pm 0.53$ & $1.37 \pm 0.07$ & $-0.31 \pm 0.13$ & 88 & $\mathrm{~K} 1 \mathrm{pBa}$ & BACCHUS \\
\hline HD 178717 & $3800 \pm 50$ & $1.00 \pm 0.50$ & 2.00 & $-0.52 \pm 0.11$ & 94 & K3.5III & $\chi^{2}$ method \\
\hline HD 199939 & $4710 \pm 9$ & $2.35 \pm 0.40$ & $1.49 \pm 0.05$ & $-0.22 \pm 0.11$ & 163 & G9III & BACCHUS \\
\hline Arcturus* & $4250 \pm 35$ & $1.50 \pm 0.06$ & $1.58 \pm 0.12$ & $-0.62 \pm 0.08$ & 1000 & K1.5III & BACCHUS \\
\hline V762 Cas* & $3800 \pm 50$ & $0.00 \pm 0.50$ & 2.00 & $-0.08 \pm 0.15$ & 110 & M3 & $\chi^{2}$ method \\
\hline
\end{tabular}

Notes. The "Remark" column specifies the method used to derive the stellar parameters (see Sect. 3). ${ }^{(*)}$ Reference objects.

discussion about the properties of the various families of extrinsic stars may be found in Jorissen (2004) for example.

Extrinsic stars being less evolved and warmer than AGB stars, their abundance analysis is much easier and they are ideal targets for the study of AGB nucleosynthesis. A recent study by Neyskens et al. (2015) is a good illustration of this; these authors propose abundance diagnostics which act as $s$-process chronometers and thermometers (respectively $\mathrm{Tc} / \mathrm{Zr}$ and $\mathrm{Nb} / \mathrm{Zr}$ ). These ratios may be used in both intrinsic and extrinsic stars, and they may also serve to distinguish extrinsic from intrinsic stars.

In the present study, we derive $\mathrm{Nb}-\mathrm{Zr}$ ratios in a representative sample of extrinsic stars to investigate the detailed operation of the $s$-process. Following the method described by Neyskens et al. (2015), our aim is to confirm that the neutron source responsible for the production of $s$-process elements is the ${ }^{13} \mathrm{C}(\alpha, \mathrm{n}){ }^{16} \mathrm{O}$ reaction, which operates in radiative layers during interpulse periods at temperatures of the order of $10^{8} \mathrm{~K}$ (e.g. Käppeler et al. 2011; Bisterzo et al. 2015), rather than the ${ }^{22} \mathrm{Ne}(\alpha, \mathrm{n}){ }^{25} \mathrm{Mg}$ reaction activated at temperatures in excess of $3.2 \times 10^{8} \mathrm{~K}$ in the convective thermal pulses of massive AGB stars $\left(M \gtrsim 4-5 M_{\odot}\right)$.

The list of target stars and the observations are described in Sect. 2. The derivation of the stellar atmospheric parameters is presented in Sect. 3. All the derived abundances are presented in Sect. 4. Light- and heavy-element abundance profiles, as well as comparison with predictions from stellar evolution and nucleosynthesis models, are presented in Sects. 6 and 7. The s-process diagnostic based on the $([\mathrm{Zr} / \mathrm{Fe}],[\mathrm{Nb} / \mathrm{Fe}])$ abundance pair is discussed in Sect. 7.4.

\section{Observational sample}

In the present paper, we have selected a sample of barium stars with photometric temperatures below $5500 \mathrm{~K}$ (Table 1) and known from former abundance analyses (e.g. Smith 1984; Allen \& Barbuy 2006; Smiljanic et al. 2007) to have large $s$-process enrichment levels. This ensures that the $s$-process signature will dominate over the pristine (presumably solar-scaled, before mass-transfer) abundance pattern. High-resolution spectra $(R \sim 86000)$ covering the wavelength range from 377.0 to $900.0 \mathrm{~nm}$ were obtained for these objects using the HERMES spectrograph (Raskin et al. 2011) mounted on the $1.2 \mathrm{~m}$ Mercator telescope at the Roque de los Muchachos Observatory, La Palma, Canary Islands. These spectroscopic data were reduced using the standard HERMES pipeline. Thanks to the HERMES long-term radial velocity monitoring of these objects (Gorlova et al. 2013), most of them are identified as radial velocity variables (the two exceptions being HD 12392 and HD 116869, for which no radial velocity monitoring is available), thus confirming their extrinsic nature. Among the many observations available covering the period 2009-2016, we selected the spectra with the best signal-to-noise ratio.

In addition to barium stars, our sample also comprises S-type stars (both extrinsic and intrinsic) and $\mathrm{M}$ stars used for comparison, all studied by Neyskens et al. (2015) using HERMES spectra as well. These stars are listed in Table 3. Moreover, in order to select good, relatively unblended lines with accurate $\log g f$ values, two reference cool giant stars were also included; one is the K1.5 giant Arcturus (Hinkle et al. 2000), and the other the M3 giant V762 Cas (HERMES spectrum). Finally, HD 28159 is a K5III star (also classified as M) initially selected to serve as a reference cool K star for our abundance analysis. However, it proved to be slightly $s$-process enriched (Sect. 4.2.3, Table 3, and Table C.1) and characterised by a variable radial velocity (Sect. 7.1). Therefore, we include it in our extrinsic star sample.

\section{Derivation of the atmospheric parameters}

Atmospheric parameters for the majority of the programme stars are derived using the BACCHUs pipeline (Masseron et al. 2016). This pipeline uses the 1D local thermodynamical equilibrium (LTE) spectrum-synthesis code Turbospectrum (Alvarez \& Plez 
Table 2. Abundances in the reference stars.

\begin{tabular}{|c|c|c|c|c|c|c|c|c|c|c|c|c|}
\hline & \multirow[b]{2}{*}{$Z$} & \multirow[b]{2}{*}{$\log \epsilon_{\odot}^{a}$} & \multicolumn{6}{|c|}{ Arcturus } & \multicolumn{4}{|c|}{ V762 Cas } \\
\hline & & & $\log \epsilon$ & $\sigma_{s}(N)$ & {$[\mathrm{X} / \mathrm{H}]^{b}$} & {$[\mathrm{X} / \mathrm{Fe}]^{b}$} & {$[\mathrm{X} / \mathrm{Fe}]^{c}$} & {$[\mathrm{X} / \mathrm{Fe}]^{d}$} & $\log \epsilon$ & $\sigma_{s}(N)$ & {$[\mathrm{X} / \mathrm{H}]$} & {$[\mathrm{X} / \mathrm{Fe}]$} \\
\hline $\begin{array}{l}\mathrm{C} \\
{ }^{12} \mathrm{C} /{ }^{13} \mathrm{C}\end{array}$ & 6 & 8.43 & $\begin{array}{l}7.7 \\
12\end{array}$ & $0.15(2)$ & -0.73 & -0.11 & - & - & $\begin{array}{r}8.40 \\
19\end{array}$ & $0.15(2)$ & -0.03 & 0.05 \\
\hline $\mathrm{N}$ & 7 & 7.83 & 7.80 & $0.06(25)$ & -0.03 & 0.59 & - & - & 7.78 & $0.10(10)$ & -0.05 & 0.03 \\
\hline $\mathrm{O}$ & 8 & 8.69 & 8.40 & $0.15(2)$ & -0.29 & 0.33 & - & - & 8.69: & - & 0.00 & 0.08 \\
\hline $\mathrm{Na}$ & 11 & 6.24 & 5.64 & $0.03(2)$ & -0.60 & 0.02 & 0.09 & 0.15 & - & - & - & - \\
\hline $\mathrm{Mg}$ & 12 & 7.60 & 7.46 & $0.06(2)$ & -0.14 & 0.48 & 0.33 & 0.37 & - & - & - & - \\
\hline $\mathrm{Fe}$ & 26 & 7.50 & 6.88 & $0.11(45)$ & -0.62 & - & -0.69 & -0.60 & 7.42 & $0.10(40)$ & -0.08 & \\
\hline $\mathrm{Rb} \mathrm{I}$ & 37 & 2.52 & - & - & - & - & - & & 2.23 & $0.22(2)$ & -0.29 & -0.21 \\
\hline $\mathrm{Sr}$ II & 38 & 2.87 & 2.20 & $-(2)$ & -0.67 & -0.05 & - & - & - & & - & - \\
\hline Y II & 39 & 2.21 & 1.48 & $0.10(4)$ & -0.73 & -0.11 & - & - & 2.15 & $0.03(6)$ & -0.06 & 0.02 \\
\hline Zr I & 40 & 2.58 & 1.84 & $0.19(2)$ & -0.74 & -0.12 & -0.09 & 0.01 & 2.40 & $0.14(2)$ & -0.18 & -0.10 \\
\hline $\mathrm{Zr}$ II & 40 & 2.58 & 1.93 & $0.07(3)$ & -0.65 & -0.03 & - & - & - & & - & - \\
\hline $\mathrm{Nb}$ II & 41 & 1.46 & - & & - & - & - & - & 1.36 & $0.10(11)$ & -0.10 & -0.02 \\
\hline Ba II & 56 & 2.18 & - & & - & - & -0.18 & -0.19 & 2.2: & - & 0.02 & 0.10 \\
\hline La II & 57 & 1.10 & 0.39 & $0.04(9)$ & -0.71 & -0.09 & -0.04 & 0.04 & 1.11 & $0.11(10)$ & 0.01 & 0.09 \\
\hline Ce II & 58 & 1.58 & 0.73 & $0.03(10)$ & -0.85 & -0.23 & - & - & 1.59 & $0.14(7)$ & 0.01 & 0.09 \\
\hline Pr II & 59 & 0.72 & 0.05 & $0.10(4)$ & -0.67 & -0.05 & - & - & 0.71 & $0.03(4)$ & -0.01 & 0.08 \\
\hline Nd II & 60 & 1.42 & 0.71 & $0.09(14)$ & -0.71 & -0.09 & - & - & 1.43 & $0.10(11)$ & 0.01 & 0.09 \\
\hline Sm II & 62 & 0.96 & 0.46 & $0.14(7)$ & -0.50 & 0.12 & - & - & 0.80 & $0.07(2)$ & -0.16 & -0.08 \\
\hline Eu II & 63 & 0.52 & 0.15 & $0.07(2)$ & -0.37 & 0.25 & 0.40 & 0.36 & 0.45 & $0.07(2)$ & -0.07 & 0.01 \\
\hline
\end{tabular}

Notes. $\sigma_{s}$ is the line-to-line scatter; $N$ the number of lines used; $\log \epsilon$ is the logarithm of the elemental abundance by number, in the scale where $\log \epsilon_{\mathrm{H}}=12$. For ${ }^{12} \mathrm{C} /{ }^{13} \mathrm{C}$ the column lists the number ratio. ${ }^{(a)}$ Asplund et al. (2009). ${ }^{(b)}$ This work. ${ }^{(c)}$ Van der Swaelmen et al. (2013). (d) Worley et al. (2009). Note: For Arcturus, Jofré et al. (2014) find $[\mathrm{Fe} / \mathrm{H}]=-0.52$.

1998; Plez 2012). The spectroscopic method for deriving the atmospheric parameters is based on ensuring consistency between the abundances derived from Fe I and Fe II lines (for $\log g$ ), between Fe lines of various excitation potentials (for $T_{\text {eff }}$ ), and between $\mathrm{Fe}$ lines of various reduced equivalent widths (for the microturbulence velocity $\xi$ ).

For a few of the coolest stars, the spectra are too blended by molecular features and BACCHUs could not converge. A $\chi^{2}$ method was used instead, selecting models which minimise $\chi^{2}$ computed from differences between observed and synthetic spectra. The spectra were split into 17 chunks of $\sim 200 \AA$ (see Table A.1) in order to correctly adjust the pseudo-continuum for each chunk. The derived parameters are presented in Table 1 along with the spectra $\mathrm{S} / \mathrm{N}$. We assume the model microturbulence of $2 \mathrm{~km} \mathrm{~s}^{-1}$, a reasonable value for such cool giants. Additional broadening is necessary to correctly reproduce the width of spectral lines; convolution by a Gaussian profile of FWHM typically $6 \mathrm{~km} \mathrm{~s}^{-1}$ was applied to account for macroturbulence and spectral resolution.

The atmospheric parameters for the $\mathrm{S}$ and $\mathrm{M}$ stars are not listed in Table 1 since they can be found in Neyskens et al. (2015).

\section{Abundance determination}

Abundances for all the elements are derived by comparing observed spectra with synthetic spectra generated by the Turbospectrum radiative transfer code using MARCS model atmospheres. We have used the same molecular lines as presented in Table B.1 of Merle et al. (2016) to derive C and N abundances. The molecular line list was taken from Masseron et al. (2014) for $\mathrm{CH}$ and from Sneden et al. (2014) for CN. The references for the other molecular line lists $\left(\mathrm{TiO}, \mathrm{SiO}, \mathrm{VO}, \mathrm{C}_{2}, \mathrm{NH}, \mathrm{OH}, \mathrm{MgH}\right.$, $\mathrm{SiH}, \mathrm{CaH}$, and $\mathrm{FeH}$ ) can be found in Gustafsson et al. (2008). The line list used for all the elements is presented in Table B.1, and is largely based upon the one used for the Gaia/ESO survey (Heiter et al. 2015). The lines are carefully selected to keep only those with negligible blending.

\subsection{Elemental abundances in the reference stars Arcturus and V762 Cas}

Table 2 lists the abundances derived in the reference stars Arcturus and V762 Cas, using the atmospheric parameters listed in Table 1. Previous abundance analyses (e.g. Maeckle et al. 1975; Ramírez \& Allende Prieto 2011; Neyskens et al. 2015) have shown that these stars are not enriched in $s$-process elements; they are used as benchmark stars to test the accuracy of our analysis. We confirm that V762 Cas has a nearly solar metallicity, with all elemental abundances within \pm 0.1 dex of the solar values, giving confidence in the adopted $\log g f$ of the selected lines. Arcturus is somewhat metal-poorer, and its abundance distribution differs from solar (e.g. Van der Swaelmen et al. 2013; Worley et al. 2009). As shown in Table 2, the abundances derived in the present paper are in good agreement with previous literature values (differences smaller than $0.1 \mathrm{dex}$ ). For the analysis of our target stars, only the lines tested in the reference stars have been used.

\subsection{Abundances in the programme stars}

The programme star abundances, along with their standard errors calculated from the line-to-line dispersion, are presented in Table C.1. 


\subsection{1. $\mathrm{C}, \mathrm{N}$, and $\mathrm{O}$}

Abundances of $\mathrm{C}, \mathrm{N}$, and $\mathrm{O}$ were obtained for all programme stars. Oxygen abundances are derived first from the [O I] line at $6300.304 \AA$, but we also use the O I resonance triplet at $7770 \AA$. This yields abundances that are consistently higher by $0.3 \mathrm{dex}$ in all the objects compared to the abundance derived from the [O I] line. This difference is usually ascribed to non-LTE (NLTE) effects strongly affecting the resonance line (Asplund et al. 2005; Amarsi et al. 2016). Hence, the $\mathrm{O}$ abundance obtained from the forbidden line is the one finally retained. For a few cool barium stars $\left(T_{\text {eff }} \leq 4000 \mathrm{~K}\right)$ for which we could detect neither the $6300.304 \AA$ [O I] line nor the high excitation line at $6363.776 \AA$, we used TiO bands instead to get the oxygen abundance.

For the coolest barium stars in our sample, the carbon abundance is obtained from the $4300 \AA \mathrm{CH} G$-band. Once the $\mathrm{C}$ abundance was known, we re-derived the $\mathrm{O}$ abundance since in cool-star atmospheres, the $\mathrm{O}$ atoms trapped in the $\mathrm{CO}$ molecule may have a strong impact on the overall $\mathrm{O}$ abundance. For the hottest barium stars in our sample, the $\mathrm{C}_{2}$ molecular lines (as listed in Table B.1 of Merle et al. 2016) are used to obtain the $C$ abundance, which is consistent with the value derived from the $\mathrm{CH} G$-band. The ${ }^{12} \mathrm{C} /{ }^{13} \mathrm{C}$ ratio is derived using ${ }^{12} \mathrm{CN}$ features at 8003.553 and $8003.910 \AA$ and ${ }^{13} \mathrm{CN}$ features at 8004.554 , 8004.728 , and $8004.781 \AA$, which are considered to be the most reliable according to Barbuy et al. (1992). We also used the ${ }^{13} \mathrm{CN}$ features at 8010.458 and $8016.429 \AA$, and checked that the abundances are consistent overall.

Finally, the nitrogen abundance is obtained in all objects from the $\mathrm{CN}$ lines available in the $7500-8400 \AA$ range.

\subsection{2. $\mathrm{Na}$ and $\mathrm{Mg}$}

Sodium abundances are mainly obtained from the Na I lines at 6154.226 and $6160.747 \AA$. Magnesium is derived using $\mathrm{Mg}_{\mathrm{I}}$ lines at 6318.717 and $6319.237 \AA$. In some objects, we were also able to use the lines at $8717.810 \AA$ and $8736.019 \AA$ in addition to the above two lines.

\subsection{3. s-process}

Abundances for all the $s$-process elements are derived from spectrum synthesis. We also considered the hyperfine splitting (hfs) for $\mathrm{Ba}, \mathrm{La}$, and $\mathrm{Eu}$, whose lines are known to be strongly affected by hfs.

$\mathrm{Rb}$. We derived $\mathrm{Rb}$ abundances in the ten coolest stars in our sample from the two $\mathrm{Rb}$ I resonance lines at 7800.259 and $7947.597 \AA$. We were not able to detect Rb I lines in the warmest targets.

Light $s$-process elements (Sr, Y, Zr). For the three first-peak $s$-process elements $\mathrm{Sr}, \mathrm{Y}$, and $\mathrm{Zr}$, lines are available for both the neutral and ionised species. Abundances for the neutral species are consistently lower than those of the ionised species. This discrepancy is discussed by many authors for barium stars (Smith 1984; Allen \& Barbuy 2006; Smiljanic et al. 2007) and S stars (Vanture et al. 2003). It is likely caused by NLTE effects affecting the minority species (here the neutral one when $T_{\text {eff }} \sim$ $4500 \mathrm{~K})$. Figure 1 indicates the yttrium dominant species according to temperature; the situation is very similar for strontium and zirconium (the singly ionised species dominating for temperatures in the range $2500 \mathrm{~K}-5500 \mathrm{~K}$ ).

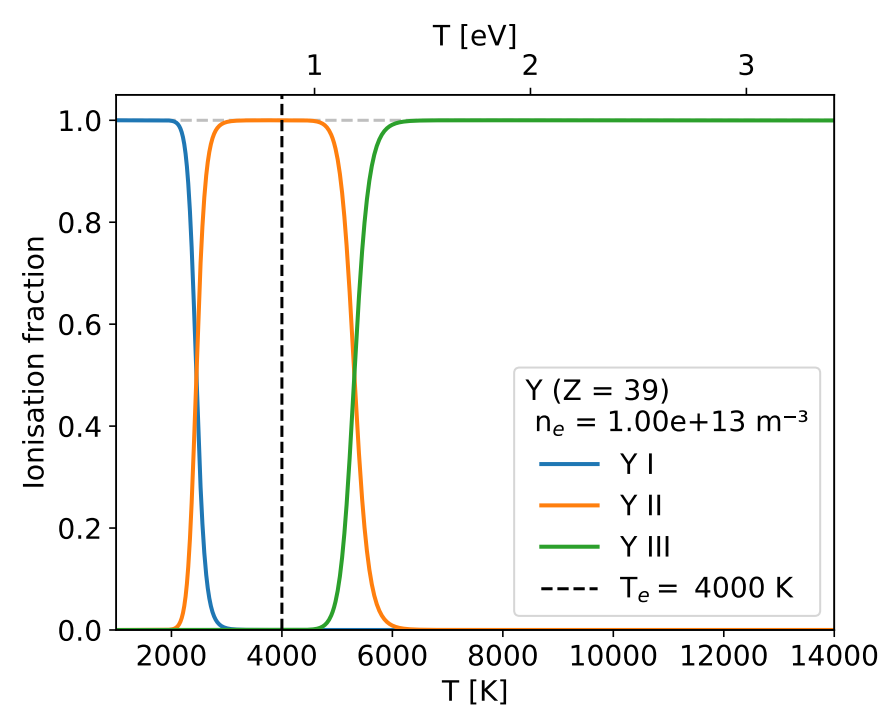

Fig. 1. Ionisation fraction vs. temperature for yttrium.

The situation for $\mathrm{Zr}$ is a bit more complex. In addition to the effect discussed above, there is a systematic discrepancy between abundances derived from the two 7819.374 and $7849.374 \AA \mathrm{Zr}$ I lines, and from many other $\mathrm{Zr}$ I lines. More precisely, the 7819.374 and $7849.374 \AA$ Zri lines systematically lead to $\mathrm{Zr}$ I abundances 0.30 dex lower than $\mathrm{Zr}$ I abundances derived from other lines with transition probabilities from Corliss \& Bozman (1962). This problem has already been noted by Neyskens et al. (2015), who decided to rely solely on abundances derived from the first pair of lines which have transition probabilities obtained from laboratory measurements (Biémont et al. 1981). Table 2 reveals that in the benchmark stars, these two $\mathrm{Zr}$ I lines yield a $\mathrm{Zr}$ abundance 0.1 dex below the solar abundance. This will be of importance in the discussion of the $\mathrm{Zr} / \mathrm{Nb}$ thermometer (Sect. 7.4).

Nb. Our selected $\mathrm{Nb}$ I lines are listed in Table B.1. Given the importance of this element for the $s$-process thermometer described in Sect. 7.4, special care has been applied in testing the adequacy of these lines to derive a reliable $\mathrm{Nb}$ abundance in the two reference stars Arcturus and V762 Cas. In Arcturus, we could not find any line sensitive to a change in the $\mathrm{Nb}$ abundance. However, it was possible to measure the $\mathrm{Nb}$ abundance in V762 Cas, yielding the solar value, similar to the situation encountered for the other $s$-process elements.

To ensure homogeneity and ease the comparison between the present sample of barium stars and the Neyskens et al. (2015) sample of $\mathrm{S}$ stars, $\mathrm{Nb}$ and $\mathrm{Zr}$ abundances in $\mathrm{S}$ stars from Neyskens et al. (2015) have been re-determined in the present paper, using the parameters of Neyskens et al. (2015) but for exactly the same lines as for the barium stars. The resulting abundances (see Table 3) are found to be in very close agreement $(\sim 0.1$ dex $)$ with those of Neyskens et al. (2015).

Heavy $s$-process elements (Ba, La, Ce, Pr, Nd). The Ba abundances listed in Table C.1 are derived from the only unsaturated line at $4524.924 \AA$.

Although there are several useful La II lines which can be used to measure the La abundance, only those with available hyperfine splitting data were considered.

To derive the $\mathrm{Ce}$ abundances, $\mathrm{Ce}$ II lines in the wavelength range 4300-6500 ̊ were used; however, these lines yield 
D. Karinkuzhi et al.: When binaries keep track of recent nucleosynthesis

Table 3. Ratios of $[\mathrm{Zr} / \mathrm{Fe}]$ and $[\mathrm{Nb} / \mathrm{Fe}]$ in the programme stars.

\begin{tabular}{|c|c|c|c|c|c|c|}
\hline Star name & {$\left[\frac{\mathrm{Fe}}{\mathrm{H}}\right]$} & $\sigma_{\left[\frac{\mathrm{Fe}}{\mathrm{H}}\right]}$ & {$\left[\frac{\mathrm{Nb}}{\mathrm{Fe}}\right]$} & $\sigma_{\left[\frac{\mathrm{Nb}}{\mathrm{Fe}}\right]}$ & {$\left[\frac{\mathrm{Zr}}{\mathrm{Fe}}\right]$} & $\sigma_{\left[\frac{\mathrm{Zr}}{\mathrm{Fe}}\right]}$ \\
\hline \multicolumn{7}{|c|}{ Barium stars } \\
\hline HD 5424 & -0.43 & 0.13 & 1.13 & 0.10 & 1.05 & 0.13 \\
\hline HD 12392 & -0.38 & 0.13 & 1.07 & 0.16 & 1.21 & 0.11 \\
\hline HD 16458 & -0.64 & 0.11 & 1.32 & 0.17 & 1.29 & 0.11 \\
\hline HD 27271 & -0.07 & 0.12 & 0.79 & 0.17 & 0.79 & 0.17 \\
\hline HD 28159 & -0.50 & 0.13 & 0.43 & 0.16 & 0.30 & 0.12 \\
\hline HD 31487 & -0.04 & 0.13 & 1.15 & 0.16 & 1.11 & 0.11 \\
\hline HD 43389 & -0.35 & 0.13 & 0.64 & 0.16 & 0.32 & 0.12 \\
\hline HD 46407 & -0.35 & 0.12 & 1.29 & 0.16 & 1.28 & 0.10 \\
\hline HD 50082 & -0.32 & 0.11 & 1.14 & 0.17 & 1.04 & 0.10 \\
\hline HD 60197 & -0.60 & 0.13 & 0.89 & 0.16 & 0.87 & 0.10 \\
\hline HD 88562 & -0.53 & 0.12 & 0.53 & 0.16 & 0.43 & 0.12 \\
\hline HD 100503 & -0.72 & 0.13 & 1.33 & 0.16 & 1.00 & 0.12 \\
\hline HD 116869 & -0.44 & 0.12 & 0.98 & 0.21 & 1.01 & 0.17 \\
\hline HD 120620 & -0.29 & 0.12 & 1.35 & 0.16 & 1.27 & 0.14 \\
\hline HD 121447 & -0.90 & 0.13 & 1.92 & 0.16 & 1.57 & 0.14 \\
\hline HD 123949 & -0.23 & 0.12 & 0.96 & 0.16 & 0.88 & 0.14 \\
\hline HD 178717 & -0.52 & 0.13 & 0.68 & 0.16 & 0.44 & 0.17 \\
\hline HD 199939 & -0.22 & 0.13 & 1.33 & 0.16 & 1.19 & 0.13 \\
\hline \multicolumn{7}{|c|}{ Extrinsic S stars } \\
\hline BD Cam & -0.03 & 0.06 & 0.61 & 0.16 & 0.78 & 0.18 \\
\hline HD 119667 & 0.01 & 0.07 & 0.39 & 0.17 & 0.59 & 0.18 \\
\hline V613 Mon & -0.26 & 0.15 & 0.40 & 0.22 & 0.66 & 0.21 \\
\hline HD 191226 & -0.28 & 0.10 & 0.19 & 0.19 & 0.35 & 0.16 \\
\hline HD 191589 & 0.01 & 0.06 & 0.20 & 0.17 & 0.29 & 0.15 \\
\hline V530 Lyr & -0.16 & 0.09 & 0.20 & 0.23 & 0.29 & 0.20 \\
\hline CPD-19 1672 & -0.01 & 0.06 & 0.28 & 0.19 & 0.41 & 0.21 \\
\hline HR 363 & -0.38 & 0.12 & 0.82 & 0.19 & 0.83 & 0.22 \\
\hline V1261 Ori & -0.22 & 0.15 & 0.80 & 0.23 & 0.85 & 0.22 \\
\hline \multicolumn{7}{|c|}{ Intrinsic S stars } \\
\hline CSS 454 & -0.40 & 0.12 & -0.07 & 0.24 & 0.80 & 0.21 \\
\hline$\sigma$ Ori & -0.45 & 0.07 & -0.04 & 0.17 & 0.45 & 0.20 \\
\hline KR CMa & -0.34 & 0.11 & 0.02 & 0.23 & 0.44 & 0.27 \\
\hline NQ Pup & -0.31 & 0.13 & -0.11 & 0.16 & 0.76 & 0.21 \\
\hline AD Cyg & -0.05 & 0.14 & 0.05 & 0.24 & 0.90 & 0.20 \\
\hline HR Peg & 0.00 & 0.06 & 0.06 & 0.08 & 0.55 & 0.18 \\
\hline AA Cam & -0.04 & 0.05 & 0.02 & 0.24 & 0.15 & 0.25 \\
\hline HIP 103476 & -0.01 & 0.06 & -0.03 & 0.16 & 0.61 & 0.18 \\
\hline \multicolumn{7}{|c|}{ M stars } \\
\hline RR UMi & -0.06 & 0.06 & -0.01 & 0.13 & -0.04 & 0.22 \\
\hline V762 Cas* & -0.08 & 0.12 & -0.02 & 0.17 & -0.10 & 0.14 \\
\hline V465 Cas & 0.03 & 0.05 & 0.03 & 0.27 & -0.13 & 0.21 \\
\hline$\mu \mathrm{Gem}$ & -0.15 & 0.06 & 0.18 & 0.18 & 0.05 & 0.20 \\
\hline$\rho$ Per & -0.06 & 0.09 & 0.10 & 0.17 & -0.09 & 0.23 \\
\hline RZ Ari & -0.22 & 0.20 & -0.15 & 0.27 & 0.02 & 0.25 \\
\hline
\end{tabular}

Notes. Data for intrinsic $\mathrm{S}$ stars and $\mathrm{M}$ stars are taken from Neyskens et al. (2015). *Reference object (see Sect. 4).

abundances that are systematically higher by about 0.3 dex than those from red Ce II lines (above $7000 \AA$ ). This may be due to non-LTE effects, which are difficult to quantify without detailed calculations.

Not many Pr lines proved useful, so we used four Pr II lines to get the Pr abundance (see Table B.1).

Since most of the violet Nd lines are blended, we used Nd II lines in the range 5200-5400 $\mathrm{A}$. For a few objects, we were also able to use clean, unblended lines at 4797.150, 4947.020, 4961.387, 5089.832, and 5132.328 $\AA$. All these lines give consistent abundances.
Table 4. Sensitivity of the abundances $\left(\Delta \log \epsilon_{\mathrm{X}}\right)$ upon variations of the atmospheric parameters.

\begin{tabular}{lrrr}
\hline \hline Element & $\begin{array}{r}\Delta T_{\text {eff }} \\
(-100) \\
(\mathrm{K})\end{array}$ & $\begin{array}{r}\Delta \log g \\
(-0.5) \\
(\mathrm{dex})\end{array}$ & $\begin{array}{r}\Delta \xi_{\mathrm{t}} \\
(-0.5) \\
\left(\mathrm{km} \mathrm{s}^{-1}\right)\end{array}$ \\
\hline $\mathrm{C}$ & -0.15 & -0.08 & 0.00 \\
$\mathrm{~N}$ & -0.30 & 0.30 & -0.10 \\
$\mathrm{O}$ & -0.15 & -0.08 & 0.00 \\
$\mathrm{Na} \mathrm{I}$ & 0.15 & 0.00 & 0.00 \\
$\mathrm{Mg}$ I & -0.45 & 0.22 & -0.05 \\
$\mathrm{Fe}$ & 0.15 & -0.20 & -0.07 \\
$\mathrm{Sr}$ I & -0.10 & 0.00 & 0.00 \\
$\mathrm{Sr}$ II & -0.15 & -0.08 & 0.02 \\
Y I & -0.23 & -0.15 & 0.10 \\
Y II & 0.03 & -0.17 & 0.09 \\
Zr I & 0.08 & -0.13 & -0.03 \\
$\mathrm{Zr} \mathrm{II}$ & 0.02 & 0.10 & 0.30 \\
$\mathrm{Nb}$ I & -0.24 & -0.16 & -0.20 \\
La II & -0.04 & -0.16 & 0.16 \\
Ce II & 0.03 & -0.11 & 0.22 \\
Pr II & 0.05 & 0.05 & 0.30 \\
Nd II & 0.05 & -0.07 & 0.20 \\
Sm II & 0.15 & 0.03 & 0.30 \\
Eu II & -0.02 & -0.22 & 0.00 \\
\hline
\end{tabular}

$r$-process elements (Sm, Eu). Good Sm lines are found in the bluer part of the spectrum, and are well fitted by the synthetic spectrum.

The Eu abundance in our objects were measured from six Eu II lines that yield consistent abundances, but the final abundances which are listed in Table C.1 are derived from the lines at $6437.640 \AA$ and $6645.134 \AA$, which yield quasi-solar abundances in the reference stars Arcturus and V762 Cas.

\subsection{Uncertainties on the abundances}

We estimated the uncertainties on all the elemental abundances $\log \epsilon$ following Eq. (2) from Johnson (2002):

$\sigma_{\log \epsilon}^{2}=\sigma_{\text {ran }}^{2}+\left(\frac{\partial \log \epsilon}{\partial T}\right)^{2} \sigma_{\mathrm{T}}^{2}+\left(\frac{\partial \log \epsilon}{\partial \log g}\right)^{2} \sigma_{\log g}^{2}+\left(\frac{\partial \log \epsilon}{\partial \xi}\right)^{2} \sigma_{\xi}^{2}$

In Eq. (1) $\sigma_{\mathrm{T}}, \sigma_{\log g}$, and $\sigma_{\xi}$ are the typical uncertainties on the atmospheric parameters, which are estimated as $\sigma_{\mathrm{T}}=50 \mathrm{~K}$, $\sigma_{\log g}=0.2 \mathrm{dex}$, and $\sigma_{\xi}=0.05 \mathrm{~km} \mathrm{~s}^{-1}$. Since uncertainties on the abundances due to the uncertainties on $[\mathrm{Fe} / \mathrm{H}]$ are very small compared to uncertainties arising from other sources, we do not consider the $\sigma_{[\mathrm{Fe} / \mathrm{H}]}$ term in our error calculations.

The partial derivatives appearing in Eq. (1) were evaluated in the specific case of HD 43389, a cool barium star with $T_{\text {eff }}=4000 \mathrm{~K}$, varying the atmospheric parameters $T_{\text {eff }}, \log g$, and microturbulence $\xi$ by $100 \mathrm{~K}, 0.5 \mathrm{dex}$, and $0.5 \mathrm{~km} \mathrm{~s}^{-1}$, respectively. The resulting changes in the abundances are presented in Table 4. Since Eq. (1) assumes that the uncertainties due to the different parameters are uncorrelated, the resulting abundance uncertainties are likely overestimated.

Finally, the random error $\sigma_{\text {ran }}$ is the line-to-line scatter. For most of the elements, we were able to use more than four lines to derive the abundances. In that case, we adopted $\sigma_{\text {ran }}=\sigma_{s} / N^{1 / 2}$, 
where $\sigma_{s}$ is the standard deviation of the abundances derived from all the $N$ lines of the considered element. It involves uncertainties caused by factors like line blending, continuum normalisation, and oscillator strength. However, for elements like $\mathrm{Zr}$ and Eu only two or three lines could be used, so that the above method had to be adapted. We first calculated $\sigma_{\mathrm{Zr} \text {,avg }}$, which is the average standard deviation on $\mathrm{Zr}$ abundances as measured on all the stars in our sample. Then $\sigma_{\mathrm{Zr} \text {,ran }}=\sigma_{\mathrm{Zr} \text {,avg }} / N^{1 / 2}$, where $N$ is the number of considered $\mathrm{Zr}$ lines. We used a similar procedure for $\sigma_{\mathrm{Eu}, \text { ran }}$. Finally the error on $[\mathrm{X} / \mathrm{Fe}]$ was calculated from $\sigma_{[\mathrm{X} / \mathrm{Fe}]}^{2}=\sigma_{\mathrm{X}}^{2}+\sigma_{\mathrm{Fe}}^{2}$.

\section{Light-element abundance analysis: $\mathrm{C},{ }^{12} \mathrm{C} /{ }^{13} \mathrm{C}, \mathrm{N}$, $\mathrm{Na}$, and $\mathrm{Mg}$}

In this section we discuss the physical conditions required for the production of the light elements $\mathrm{C}, \mathrm{N}, \mathrm{F}, \mathrm{Na}$, and $\mathrm{Mg}$.

Except for HD 120620 (with ${ }^{12} \mathrm{C} /{ }^{13} \mathrm{C}=90$ ), all barium stars have ${ }^{12} \mathrm{C} /{ }^{13} \mathrm{C}$ values lower than 20 (Fig. 2), a feature already noted by Tomkin \& Lambert (1979), Sneden et al. (1981), Smith (1984), Harris et al. (1985), and Barbuy et al. (1992). This is indeed expected if matter processed by the $\mathrm{CN}$ cycle and brought to the surface by the first dredge-up dominates over the ${ }^{12} \mathrm{C}$ pollution from the AGB companion. In most of the programme stars, we find a nitrogen overabundance $[\mathrm{N} / \mathrm{Fe}] \sim 0.4 \operatorname{dex}$ (top panel of Fig. 2), in agreement with the target stars being subject to efficient mixing (Lambert \& Ries 1981). The situation observed in barium stars (namely ${ }^{12} \mathrm{C} /{ }^{13} \mathrm{C}<20$ and $[\mathrm{N} / \mathrm{Fe}] \sim$ 0.4 dex) matches the predictions of models combining the first dredge-up on the red giant branch with the subsequent thermohaline mixing occurring at the "bump" (Eggleton et al. 2008).

In some barium stars, however (HD 43389, HD 60197, HD 100503, and to a lesser extent HD 121447), $\mathrm{N}$ overabundances as high as $\sim 1.2 \mathrm{dex}$ are measured. Nitrogen enrichments in barium stars have already been reported by Barbuy et al. (1992), Allen \& Barbuy (2006), and Merle et al. (2016), although generally not to such extreme levels. This situation may still be accounted for by the previous models, provided the barium star was formerly enriched by ${ }^{14} \mathrm{~N}$ from a massive AGB companion experiencing hot-bottom burning (HBB; e.g. Boothroyd et al. 1995, and references therein) where hydrogen burns at the bottom of the convective envelope.

A further confirmation of this ${ }^{14} \mathrm{~N}$ pollution is given in the top panel of Fig. 3, which reveals that the four N-enriched objects have distinct $\mathrm{C}$ abundances (see bottom panel of Fig. 3) but similar $\mathrm{C}+\mathrm{N}$ abundances, revealing that $\mathrm{CN}$-processing might indeed have operated. Interestingly, the $\mathrm{C}+\mathrm{N}$ abundances in these four peculiar objects are globally higher than those of the bulk of barium stars (top panel of Fig. 3).

The bottom panels of Fig. 3 compare the $\mathrm{C}$ abundance in barium stars with the trend present in the Galactic discs derived by Bensby \& Feltzing (2006) and Suarez-Andres et al. (2017). Both Galactic determinations of $[\mathrm{C} / \mathrm{Fe}]$ as a function of $[\mathrm{Fe} / \mathrm{H}]$ yield a broadly consistent picture. We note, however, that Reddy et al. (2006) find a significant increase in $[\mathrm{C} / \mathrm{Fe}]$ with decreasing $[\mathrm{Fe} / \mathrm{H}]$, contrarily to Bensby \& Feltzing (2006) and Suarez-Andres et al. (2017). The majority of barium stars in our sample clearly exhibit carbon overabundances with respect to the Galactic trends. This is consistent with stellar evolution considerations since the matter accreted by the barium star was processed by the third dredge-up in the donor star.

The partial mixing of protons inside the C-rich layers of the H-He intershell region of AGB stars can be responsible not

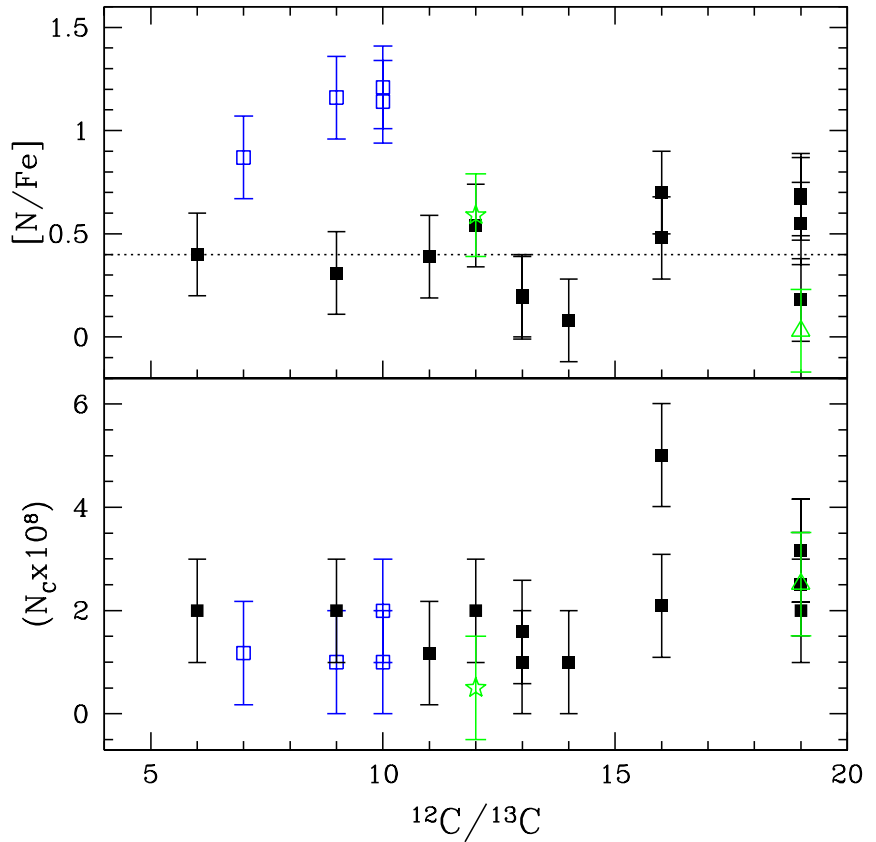

Fig. 2. Top panel: $[\mathrm{N} / \mathrm{Fe}]$ as a function of ${ }^{12} \mathrm{C} /{ }^{13} \mathrm{C}$, represented for our programme stars with full and open blue squares; open blue squares specifically identify the N-enriched objects (HD 43389, HD60197, HD 100503, and to a lesser extent HD 121447). Arcturus (green star) and V762 Cas (green triangle) are also shown for comparison. The horizontal dotted line shows the abundance ratio expected in red giant stars after the mixing processes occurring along the giant branch (Eggleton et al. 2008). Bottom panel: Same as the top panel for the C abundance (by number, in the scale where $\log \epsilon_{\mathrm{H}}=12$ ).

only for an efficient $s$-process nucleosynthesis but also for a significant enrichment in ${ }^{19} \mathrm{~F}$ (Goriely \& Mowlavi 2000). Fluorine abundances have been determined in only three barium stars so far (HD 121447, HD 123396, and HD 178717, the first and the last belong to our sample; Jorissen et al. 1992; Alves-Brito et al. 2011); they all reveal fluorine enrichments (at the level $0.4 \leq$ $[\mathrm{F} / \mathrm{O}] \leq 0.7)$. Even though the very high $\mathrm{F}$ abundances found in carbon stars in the early work of Jorissen et al. (1992) were later revised downwards by Abia et al. (2009, 2010, 2015; due to blends of $\mathrm{CN}$ and $\mathrm{C}_{2}$ lines with the HF lines), these stars still remain F-rich (see Fig. 3 from Pilachowski \& Pace 2015).

A sodium enrichment may also be expected in AGB stars (and thus in barium stars) through the $\mathrm{NeNa}$ chain of proton captures (Goriely \& Mowlavi 2000). Such a Na enrichment in barium stars has already been noted by de Castro et al. (2016), and is observed in some of our barium stars as well (Fig. 4). It is explained by our models (Figs. 9 and 10) except for HD 121447 whose interpretation is quite challenging (see discussion below).

The four lowest metallicity $([\mathrm{Fe} / \mathrm{H}] \leq-0.6)$ barium stars in our sample (HD 16458, HD 60197, HD 100503, and HD 121447) are all very Na-enriched ([Na/Fe] $>0.5)$, as shown in Figs. 4 and 5. The last three are also enriched in nitrogen, along with HD 43389 (of higher metallicity: $[\mathrm{Fe} / \mathrm{H}]=-0.35$ ). Non-LTE effects are known to affect the Na lines; the corrections (to be applied on LTE abundances) are smaller than $\Delta_{\mathrm{NLTE}}=-0.15$ dex considering the barium star parameter range (Lind et al. 2011, and the INSPECT project). This correction would not be sufficient to reconcile the most-enriched $\mathrm{Na}$ abundances in barium stars with those of disc stars.

Interestingly, at the very high temperatures achieved at the base of the convective envelope of super-AGB stars $\left(T_{\text {env }}>\right.$ 


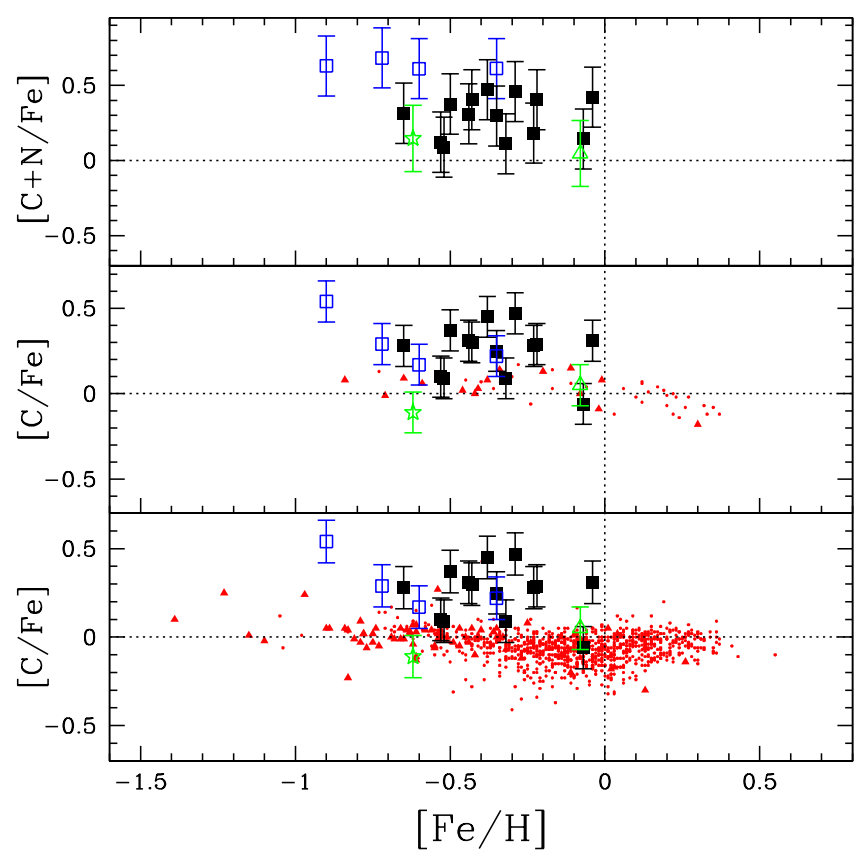

Fig. 3. Abundances of $\mathrm{C}$ (bottom and middle panels) and $\mathrm{C}+\mathrm{N}$ (top panel) in our programme stars, as a function of metallicity (symbols defined in Fig. 2). The $\mathrm{C}$ abundances of thick disc (red triangles) and thin disc (red dots) stars from Bensby \& Feltzing (2006; middle panel) and Suarez-Andres et al. (2017; bottom panel) are also shown to outline the Galactic trend.

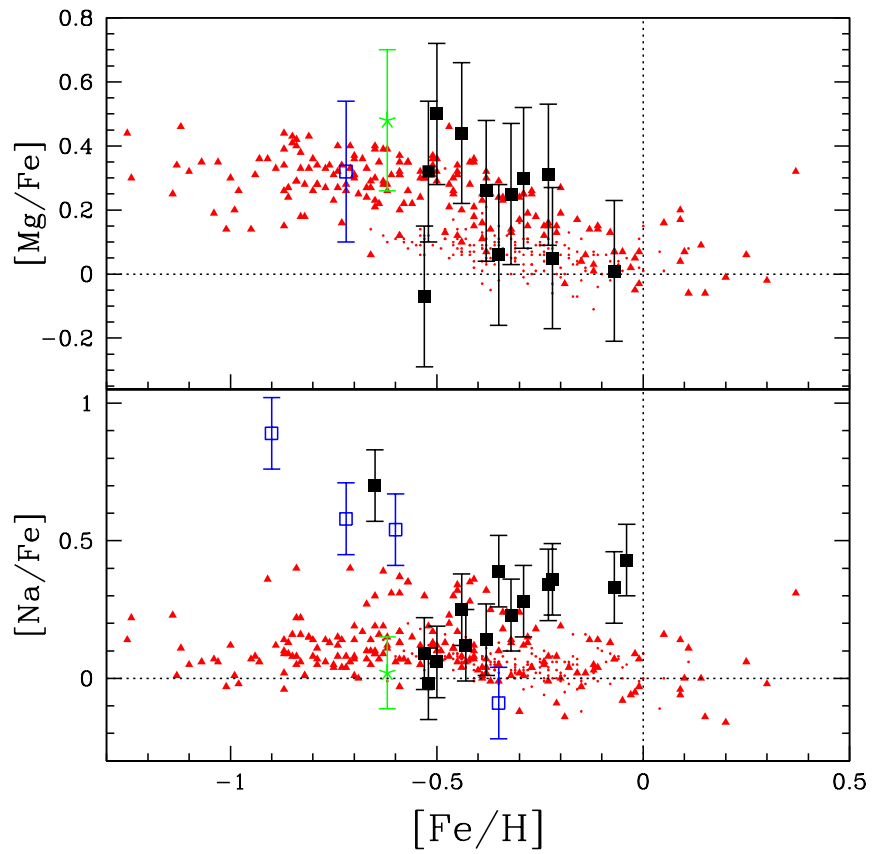

Fig. 4. Abundances of $\mathrm{Na}$ and $\mathrm{Mg}$ of our programme stars (symbols defined in Fig. 2) along with Arcturus (green star), compared with thin disc stars (red dots; Bensby et al. 2005; Reddy et al. 2003). Thick disc stars (red triangles; Bensby et al. 2005; Reddy et al. 2006) are also shown for comparison.

$10^{8} \mathrm{~K}$ ), N can be substantially produced by HBB (Doherty et al. 2014a). These four $\mathrm{N}$-enriched objects show variations in the $\mathrm{C}$ abundance from star to star (see bottom panel of Fig. 3), but similar $\mathrm{C}+\mathrm{N}$ abundances (top panel of Fig. 3), revealing that $\mathrm{CN}$ processing has probably occurred.

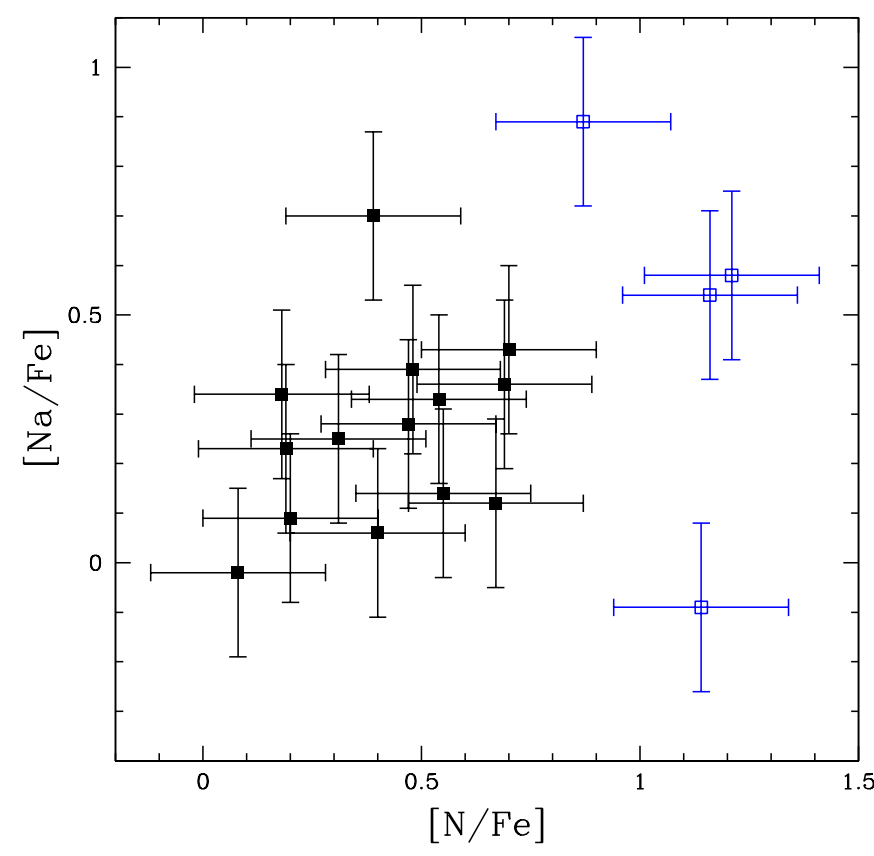

Fig. 5. Barium stars in the $([\mathrm{Na} / \mathrm{Fe}],[\mathrm{N} / \mathrm{Fe}])$ plane. The four $\mathrm{N}$-rich objects HD 43389, HD 60197, HD 100503, and HD 121447 are shown as blue open squares.

Above $[\mathrm{Fe} / \mathrm{H}] \sim-0.6$, the predictions of Doherty et al. (2014a) for super-AGB stars agree with the observed decrease in $\mathrm{Na}$ abundance with metallicity, but they remain at odds with the trend shown by the four most metal-poor stars in our sample (with enhanced Na). As noted by Doherty et al. (2014b), a very high mass-loss rate during the AGB phase would minimise the ${ }^{23} \mathrm{Na}$ destruction [through ${ }^{23} \mathrm{Na}(\mathrm{p}, \alpha){ }^{20} \mathrm{Ne}$ and ${ }^{23} \mathrm{Na}(\mathrm{p}, \gamma){ }^{24} \mathrm{Mg}$ ], thus preserving a relatively high $\mathrm{Na}$ abundance. Interestingly enough, we note that in the present sample, the star with the highest $[\mathrm{Na} / \mathrm{Fe}]$ abundance is HD 121447, which is also the barium star with the third shortest orbital period $(185.7 \mathrm{~d})$. If the orbital period was as short at the end of the TP-AGB phase of the donor star, this star could well have suffered from a strongly enhanced mass transfer, as proposed for example by Tout \& Eggleton (1988), thus shortening the time spent on the AGB. Combined with the Doherty et al. (2014b) argument above, this could possibly account for the high $\mathrm{Na}$ abundance observed in this star.

Finally, if the $s$-process nucleosynthesis is taking place in hot thermal pulses, $\mathrm{Mg}$ is expected to be overproduced as a consequence of $\alpha$-captures by ${ }^{22} \mathrm{Ne}$. This signature is not observed in our barium stars since they show a level of $\mathrm{Mg}$ enrichment compatible with the Galactic trend (Fig. 4), which further discards ${ }^{22} \mathrm{Ne}(\alpha, \mathrm{n})^{25} \mathrm{Mg}$ as the main neutron source for the $s$ process. de Castro et al. (2016) report measurements in barium stars of $[\mathrm{Mg} / \mathrm{Fe}]$ (and more generally of $[\alpha / \mathrm{Fe}]$ ) higher than local field giant stars; these barium stars could be transition objects between the thin and thick disc, or be thick disc stars.

To conclude, the most salient feature emerging from the present study of light elements in barium stars is the suggestion offered by the $\mathrm{N}$ and $\mathrm{Na}$ overabundances that some may have been polluted by rather massive AGB stars undergoing HBB. However, we see in Sect. 7.1 that this conclusion is not supported by the $s$-process abundance pattern, which forbids AGB stars more massive than $3 M_{\odot}$ from being at the origin of the pollution of barium stars. 
In Sect. 7.2 we connect the light-element abundances discussed so far with abundances of $s$-process elements and compare the results with stellar model and nucleosynthesis predictions that are described in the next section.

\section{STAREVOL models}

Asymptotic giant branch models have been computed with the STAREVOL code (Siess et al. 2000; Siess 2006) using an extended $s$-process reaction network of 411 species and the same input physics as described in Goriely \& Siess (2018). In our computations, we use the standard mixing length theory with $\alpha=1.75$. We take into account the change in opacity due to the formation of molecules when the star becomes carbon rich, as prescribed by Marigo (2002). The reference solar composition is given by Asplund et al. (2009) which corresponds to a metallicity $Z=0.0134$. For the lower metallicity models, we assume a solar-scaled composition without $\alpha$-element enhancement. For the mass-loss rate, we consider the Reimers (1975) prescription with $\eta_{\mathrm{R}}=0.4$ from the main sequence up to the beginning of the AGB and then switch to the Vassiliadis \& Wood (1993) rate. Dedicated models have been computed for $[\mathrm{Fe} / \mathrm{H}]$ between -1 and 0 , with steps of 0.1 , for masses of 2 and $3 M_{\odot}$.

In the present simulations, a diffusion equation is used to simulate the partial mixing of protons in the C-rich layers at the time of the third dredge-up. Following the formalism of Eq. (9) of Goriely \& Siess (2018), the diffusive mixing (DM) parameters adopted in our calculations are $D_{\min }=10^{9} \mathrm{~cm}^{2} \mathrm{~s}^{-1}$ and $p=5$, where $D_{\min }$ is the value of the diffusion coefficient at the innermost boundary of the diffusive region and $p$ is an additional free parameter defining the slope of the exponential decrease in the overshoot diffusion coefficient with depth. This formalism is actually used for 2 and $3 M_{\odot}$ stars only. In 4 and $5 M_{\odot}$ stars, the high temperature at the base of the convective envelope requires a coupling of mixing and burning, which is not yet included in our simulations. Nevertheless, it was shown in Goriely \& Siess (2004) that when HBB is effective, the protons are burnt on-the-fly leading to an overlap of ${ }^{13} \mathrm{C}$ with (neutron poison) ${ }^{14} \mathrm{~N}$, resulting in a very inefficient $s$-process nucleosynthesis. We note, however, that an $s$-production by ${ }^{22} \mathrm{Ne}$ can still occur in such stars.

\section{Heavy-element abundance analysis}

\section{1. s-process abundance pattern}

Comparison between the observed and predicted $s$-process abundance patterns for various stellar masses is shown in Figs. 6 to 8 . The pulse number corresponding to the model that best reproduces the overall surface enrichment is indicated for each observed star. A good agreement is systematically obtained with the predictions corresponding to $2 M_{\odot}$ (red line) or $3 M_{\odot}$ (blue line) stars, with metallicities matching the measured values. This initial mass of the polluting AGB stars has to be higher than the mass of the current Ba star (because the polluting star evolved first), but this effect might be softened because the current barium star accreted mass (up to a few tenths of $M_{\odot}$ from the simulations). In any case, the masses of the current barium stars derived from their position in the Hertzsprung-Russell (HR) diagram $\left(M=1.0-2.7 M_{\odot}\right.$, Table 5 and Sect. 7.4.3) is compatible with a $2-3 M_{\odot}$ companion.

Abundances from more massive AGB stars (4-5 $M_{\odot}$ ) have systematically lower [hs/ls] ratios than those displayed in Fig. 6, where ls and hs denote the average abundance of $s$-process elements belonging to first peak ( $\mathrm{Sr}, \mathrm{Y}, \mathrm{Zr}$ ) and second peak ( $\mathrm{Ba}$, $\mathrm{La}, \mathrm{Ce})$, respectively. This results from the fact that the $s$-process is taking place in the convective pulse rather in a radiative layer (see e.g. Goriely \& Siess 2018).

Two stars deserve a special discussion. The first, HD 28159, is the least $s$-process enriched star in our sample. As already mentioned in Sect. 2, this K5III giant was initially selected to serve as a solar-scaled composition reference object. Its abundance distribution (Fig. 7) reveals that it is slightly enriched in $\mathrm{La}([\mathrm{La} / \mathrm{Fe}]=0.87 \pm 0.12)$ and to a lesser extent in $\mathrm{Zr}, \mathrm{Ce}, \mathrm{Pr}$, $\mathrm{Nd}, \mathrm{Sm}$, and $\mathrm{Eu}$. The La abundance can hardly be reproduced, even considering its error bar, with the $3 M_{\odot}$ model predictions, which moreover overestimates by $\sim 0.4$ dex the abundances of $\mathrm{Ce}, \mathrm{Pr}$, and $\mathrm{Nd}$ (Fig. 7). To reconcile such a modest [hs/ls] ratio with the metallicity of the $\operatorname{star}([\mathrm{Fe} / \mathrm{H}]=-0.5)$ is difficult. Interestingly enough, this object is probably a binary. This conclusion is not drawn from the radial velocity of $-6.5( \pm 3) \mathrm{km} \mathrm{s}^{-1}$ quoted (without associated date) by Wilson (1953), but rather from the two available HERMES spectra, which lead to the following radial velocities: $-7.745 \pm 0.007 \mathrm{~km} \mathrm{~s}^{-1}$ on JD 2455508.70 ; $-8.177 \pm 0.014 \mathrm{~km} \mathrm{~s}^{-1}$ on JD 2456619.57 . This object is thus a newly discovered $s$-process enriched, probably extrinsic star. Its hs abundance profile is difficult to reconcile with that of the other extrinsic stars in our sample and with the model predictions, but its $\mathrm{Zr}$ and $\mathrm{Nb}$ abundances perfectly follow the trend expected for extrinsic stars (see discussion in Sect 7.4 and Fig. 14).

The second star, HD 121447, on the contrary, is the most metal-poor $([\mathrm{Fe} / \mathrm{H}]=-0.9)$ and the most $s$-process enriched barium star in our sample. It is also the coolest known barium star with the third shortest orbital period $(P=185.7 \mathrm{~d})$. It shows several peculiarities including ellipsoidal photometric variations and a rotation rate not synchronised with the orbital motion, though orbital circularisation has been achieved (Jorissen et al. 1995). Scattering on some obscuring material, possibly trapped around the WD companion, could also potentially account for the photometric variability. From the analysis of its light and velocity curves, Jorissen et al. (1995) estimate a mass of 1.51.7 $M_{\odot}$ consistent with the range we derive in Sect. 7.4.3 from its location in the HR diagram. The abundance distribution (probing the initial mass of the primary, i.e. of the current white dwarf) is reproduced well by a $2 M_{\odot}$ model, as shown in Fig. 8 , and is consistent with the primary being initially more massive than the secondary, and evolving more quickly.

\subsection{Trends between light and heavy elements}

Figures 9 and 10 compare the measured average $s$-process abundance $[\mathrm{s} / \mathrm{Fe}]$ and the light elements $\mathrm{C}, \mathrm{N}, \mathrm{Na}$, and $\mathrm{Mg}$ with the predictions from 2 and $3 M_{\odot}$ models of various metallicities.

The predicted carbon abundances are generally too high with respect to the observed ones. This may simply be due to the fact that dilution of the AGB matter in the barium star envelope has not been considered in Figs. 9 and 10, and that barium stars are post-first dredge-up. Both processes will bring the predicted carbon abundance down, in better agreement with the observed value.

The above arguments, however, cannot explain the highest measured $\mathrm{N}$ overabundances. Figure 11 reveals that all N-rich stars are characterised by high [hs/ls] ratios pointing toward an efficient $s$-process. Therefore, the $\mathrm{N}$ enrichment cannot be attributed to hot-bottom burning occurring in massive AGB stars, since as discussed in Sects. 7.1 and 7.5, they are characterised by low [hs/ls] ratios and high Rb abundances. Similarly, large 
D. Karinkuzhi et al.: When binaries keep track of recent nucleosynthesis
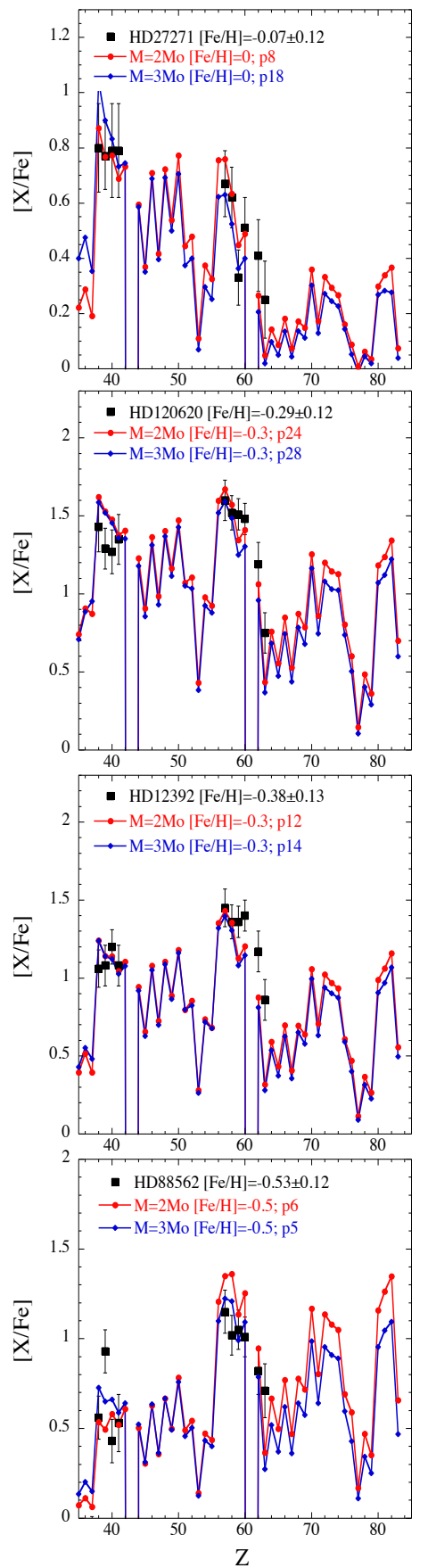
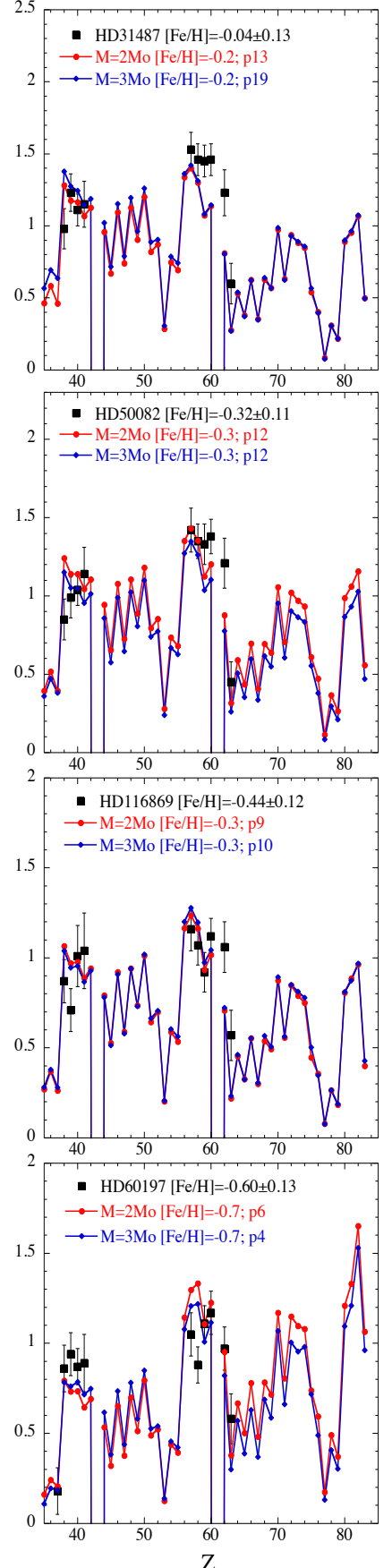
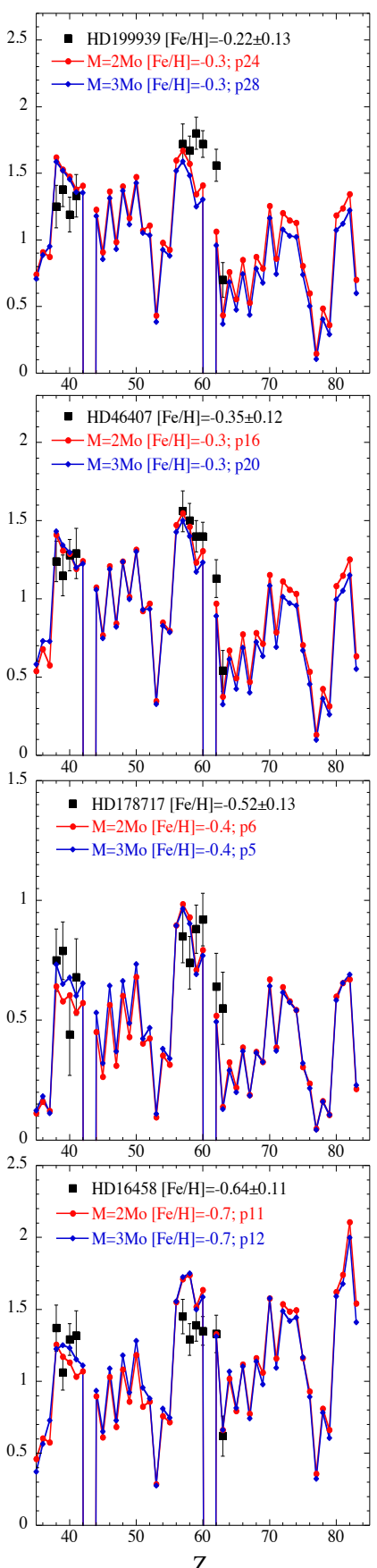
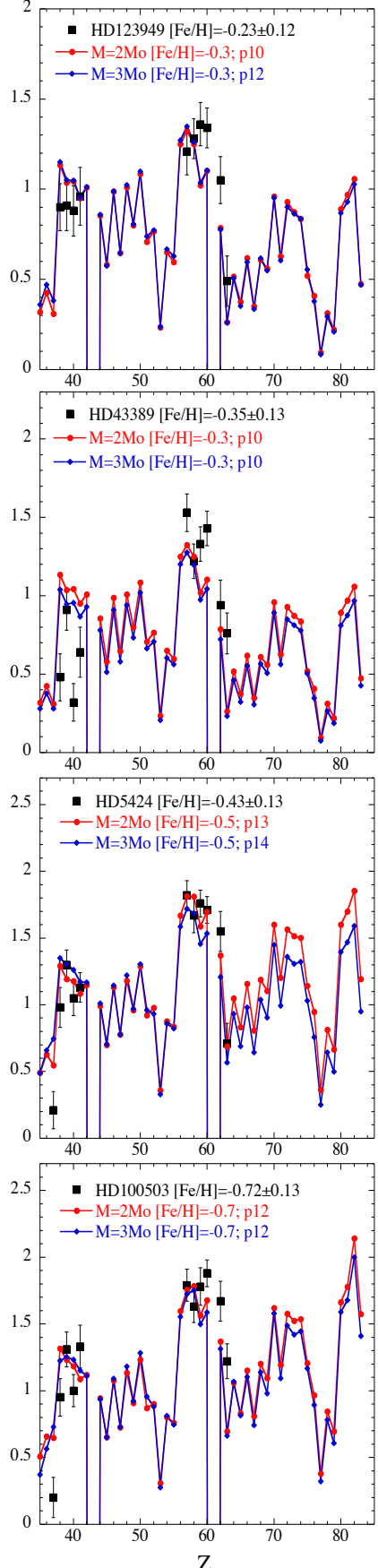

Fig. 6. Observed $s$-process abundance patterns (black squares with error bars) compared with the predictions for $2 M_{\odot}$ (red line) and $3 M_{\odot}$ (blue line) models with the diffusive mixing scheme. The pulse number is quoted in the label as "p". The stars are ordered by decreasing model metallicity.

envelope pollutions in carbon and nitrogen are expected from low-mass metal-poor AGB stars experiencing H-ingestion events (Campbell \& Lattanzio 2008), but the required metallicity is too low and incompatible with those measured in the N-rich barium stars, whose origin thus remains a mystery.

Finally, the observed $\mathrm{Na}$ abundances are well reproduced globally, except for the star most enriched in Na. Again, the dilution factor has not been considered.

The $\mathrm{Mg}$ abundances are not well reproduced by the predictions likely because the corresponding models did not start from initial abundances matching the Galactic trend displayed in Fig. 4.

\subsection{Chemical overabundances and orbital elements}

Mass transfer being responsible for the chemical peculiarities of barium stars, a correlation is expected between the orbital period and the overabundance level. Figure 12 displays the bariumstar abundances of $s$ - and $r$-process elements derived in the present study as a function of their orbital period (Appendix A of Van der Swaelmen et al. 2017). A loose correlation is indeed present, maybe more clearly seen for $\mathrm{Zr}, \mathrm{Ba}$, and $\mathrm{Nd}$, pointing to a lower enrichment when the orbital period is longer. No clear metallicity modulation (colour-coded in Fig. 12) is observed, though the small number of investigated stars pre- 


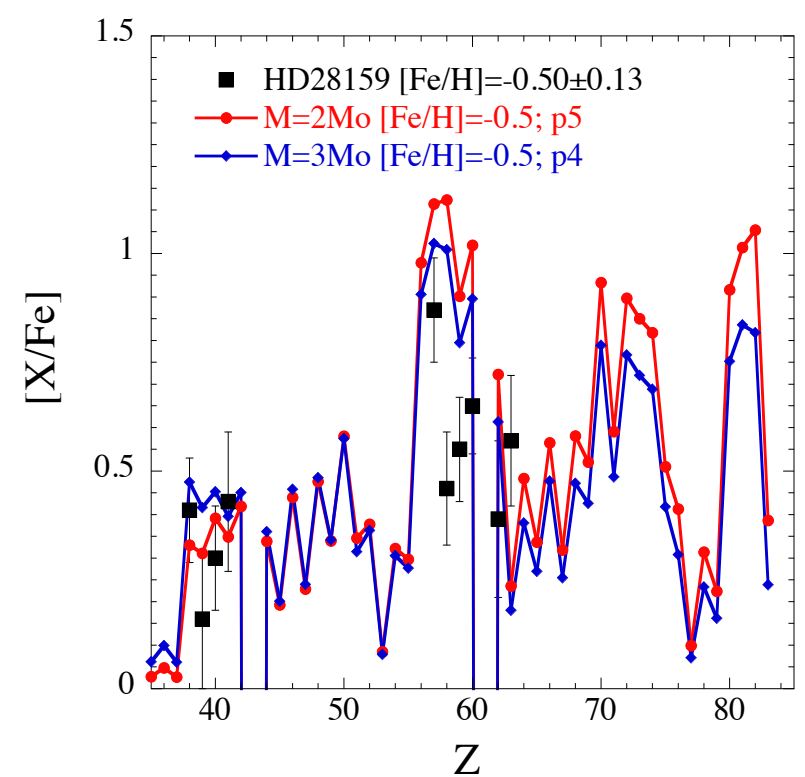

Fig. 7. Same as Fig. 6, but for HD 28159. Our analysis reveals that this object, initially classified as a normal giant (K5III), is slightly $s$-process enriched. The agreement with the predictions for a $2 M_{\odot}$ (red line) and $3 M_{\odot}$ (blue line) model with the diffusive mixing scheme is not satisfactory.

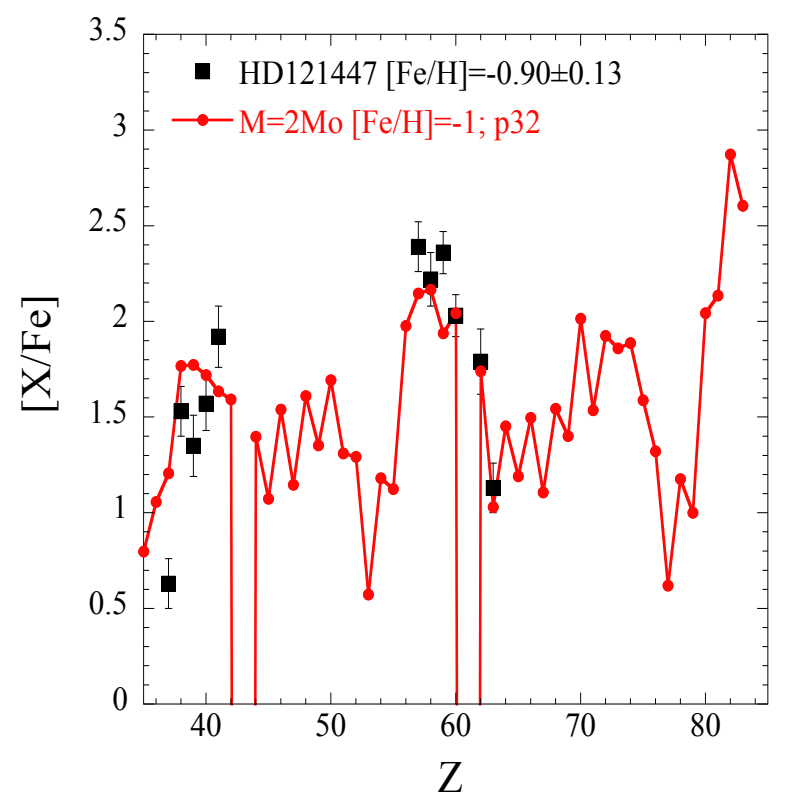

Fig. 8. Same as Fig. 6, but for HD 121447, the most extreme barium star in our sample, compared with the predictions for a $2 M_{\odot}$ (red line) model with the diffusive mixing scheme.

vents us from drawing any firm conclusion on a possible impact of the stellar metallicity on the $s$-process overabundance level.

The overabundance-period trend is somewhat clearer when considering an average of eight measured $s$-process elements as indicator (top panel of Fig. 13). This correlation remains when considering the periastron distance instead of the orbital period. Another interesting aspect to be discussed is the impact of the orbital eccentricity. The most eccentric barium stars $(e>0.3)$ tend to be among the least $s$-process enriched (red points in Fig. 13). When occurring in a system characterised by an eccentric orbit, the efficiency of the mass transfer, being probably

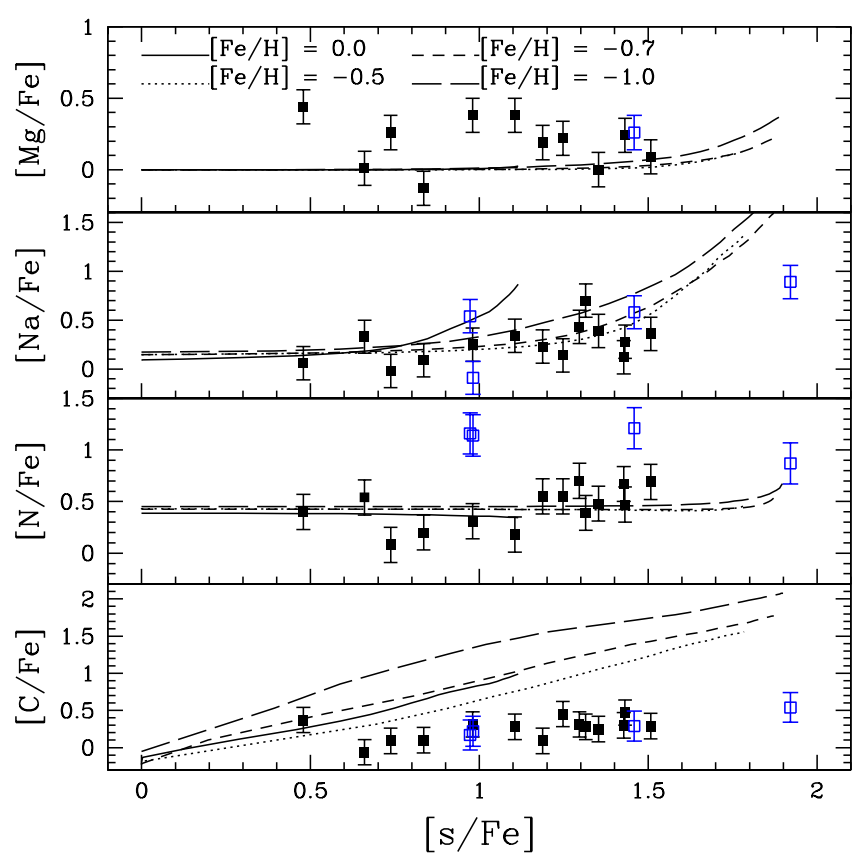

Fig. 9. Abundances of $\mathrm{C}, \mathrm{N}, \mathrm{Na}$, and $\mathrm{Mg}$ (from bottom to top) in our programme stars are shown as a function of the $s$-process abundances $[\mathrm{s} / \mathrm{Fe}]$, where $[\mathrm{s} / \mathrm{Fe}]=([\mathrm{Sr} / \mathrm{Fe}]+[\mathrm{Y} / \mathrm{Fe}]+[\mathrm{Zr} / \mathrm{Fe}]+[\mathrm{Nb} / \mathrm{Fe}]+[\mathrm{La} / \mathrm{Fe}]$ $+[\mathrm{Ce} / \mathrm{Fe}]+[\mathrm{Pr} / \mathrm{Fe}]+[\mathrm{Nd} / \mathrm{Fe}]) / 8$. Predicted values from $2 M_{\odot}$ models for different metallicities are shown for comparison (symbols defined in Fig. 2).

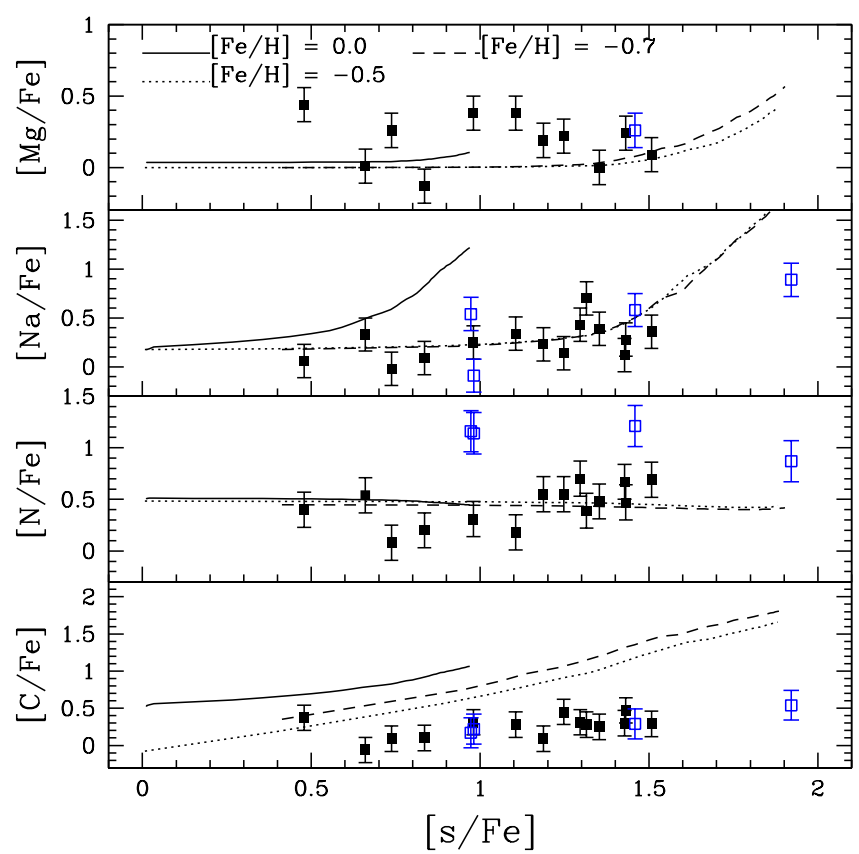

Fig. 10. Same as Fig. 9, but for $3 M_{\odot}$ models.

intermittent, could be less than when occurring continuously along a circular orbit.

\subsection{The $\mathrm{Zr}-\mathrm{Nb}$ pair as $s$-process thermometer}

\subsubsection{Method}

The aim of this section is to apply the method described in Neyskens et al. (2015) to a larger sample of stars characterised 


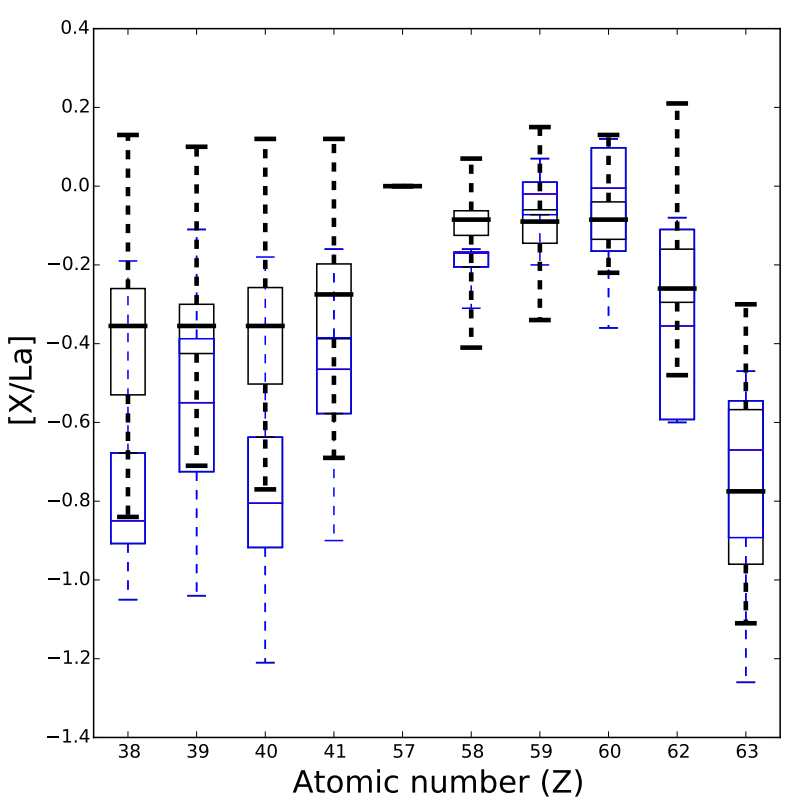

Fig. 11. Heavy-element abundances in the programme stars normalised to their La abundance $(Z=57)$. The blue boxes refer to the four $\mathrm{N}$-rich stars, whereas black boxes depict the range covered by all the other stars. The bottom and top of the boxes refer to the 25th and 75 th percentiles in the considered elemental abundance. The light and heavy $s$-elements (ls and hs) are delineated by the atomic number $Z=57$.

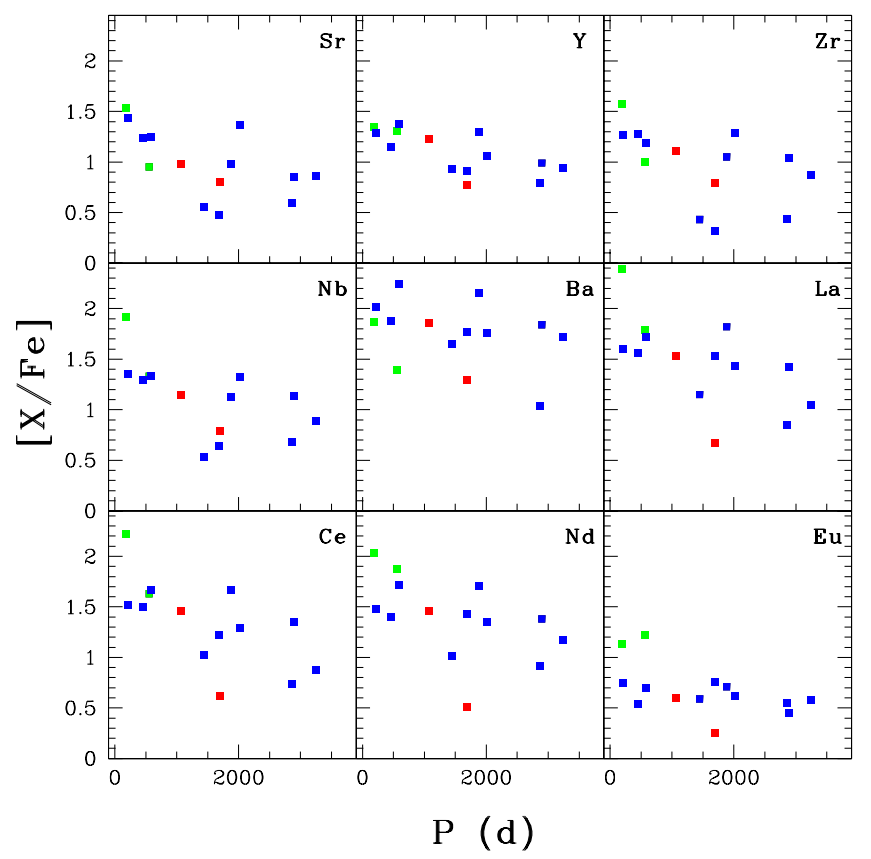

Fig. 12. Abundance of heavy elements (as labelled) as a function of the orbital period. The metallicity is coded as follows: green: $[\mathrm{Fe} / \mathrm{H}]$ $\leq-0.65$; blue: $-0.65<[\mathrm{Fe} / \mathrm{H}] \leq-0.1$; red: $[\mathrm{Fe} / \mathrm{H}]>-0.1$.

by high levels of $s$-process enrichments. We show that for lowmass stars $\left(M \leq 3 M_{\odot}\right)$ a simple straight line drawn in the $([\mathrm{Zr} / \mathrm{Fe}],[\mathrm{Nb} / \mathrm{Fe}])$ diagram provides an estimate of the $s$-process operation temperature, whereas for higher mass $\operatorname{stars}(M \geq$ $4 M_{\odot}$ ), this diagram keeps a predictive power for the $s$-process operation temperature, but the relation is more complex (see in Sect. 7.4.2).

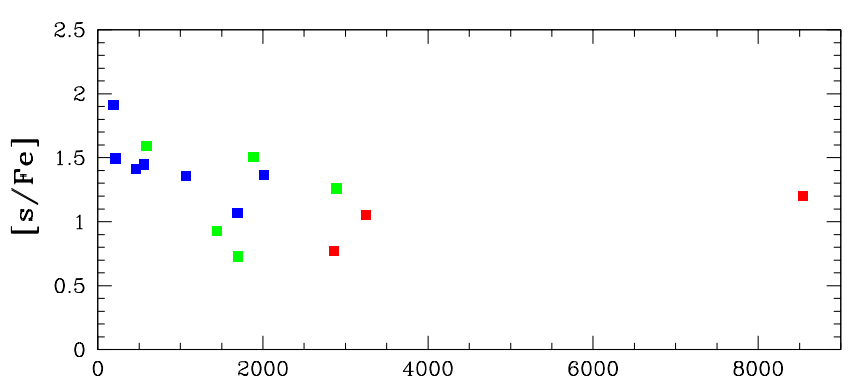

$P(d)$

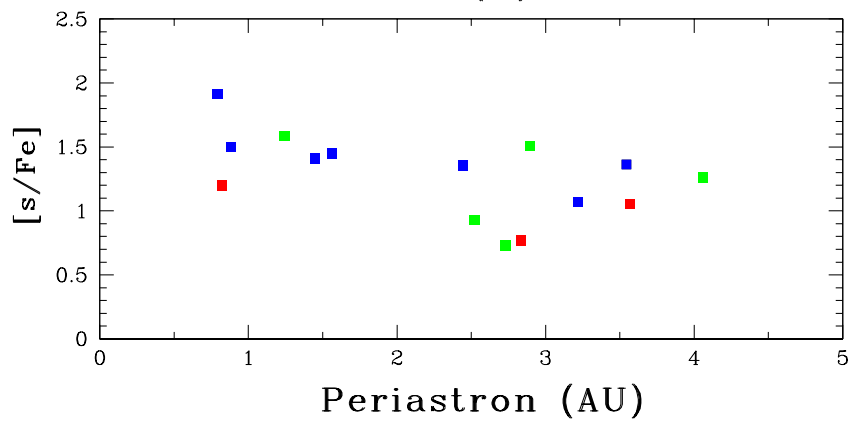

Fig. 13. Mean $s$-process abundance ( $s$ is an average of the $\mathrm{Sr}, \mathrm{Y}, \mathrm{Zr}, \mathrm{Nb}$, $\mathrm{Ba}, \mathrm{La}, \mathrm{Ce}, \mathrm{Pr}, \mathrm{Nd}$ abundances) as a function of the orbital period (top panel) and of the periastron distance (bottom panel), assuming the total mass of the binary system is $2 M_{\odot}$. The eccentricity is coded as follows: red: $e>0.3$; green: $0.1<e \leq 0.3$; blue: $e \leq 0.1$.

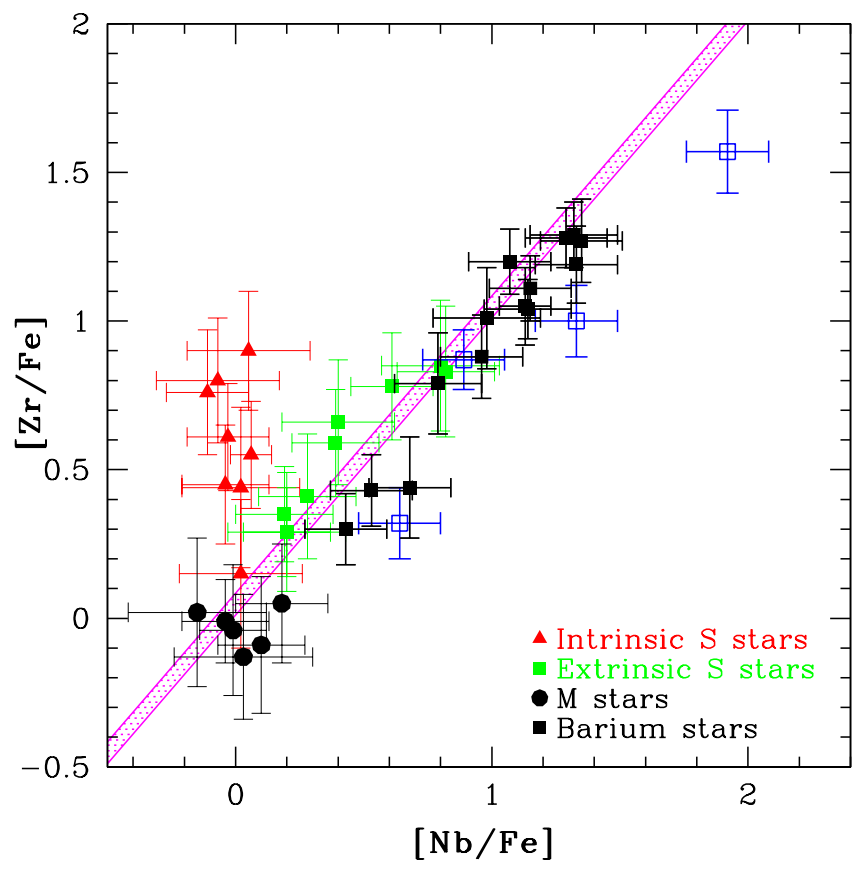

Fig. 14. Distribution of $[\mathrm{Zr} / \mathrm{Fe}]$ vs. $[\mathrm{Nb} / \mathrm{Fe}]$ for our target stars. Abundances of intrinsic S stars and M stars are from Neyskens et al. (2015). The abundances of extrinsic $\mathrm{S}$ stars and barium stars are from this work. The open blue squares refer to the four $\mathrm{N}$-enriched barium stars (HD 43389, HD 60197, HD 100503, and HD 121447 in order of increasing value of $[\mathrm{Nb} / \mathrm{Fe}])$. HD 28159 , a star previously known as K0III and which proved to be slightly enriched in $s$-process (see Sect. 7.1) is located at $([\mathrm{Nb} / \mathrm{Fe}],[\mathrm{Zr} / \mathrm{Fe}])=(0.43,0.30)$. The magenta shaded band indicates the expected location of stars polluted by material resulting from the $s$-process operating at temperatures between 1.0 (upper line) and $3.0 \times 10^{8} \mathrm{~K}$ (lower line). These simple predictions assume that the chain of neutron captures along the $\mathrm{Zr}$ isotopes is in local equilibrium. 
The method compares the $[\mathrm{Zr} / \mathrm{Fe}]$ and $[\mathrm{Nb} / \mathrm{Fe}]$ abundances in intrinsic stars (red symbols in Fig. 14), which are still on the AGB producing ${ }^{93} \mathrm{Zr}$, with abundances in the older extrinsic stars that accreted the AGB material. In these extrinsic stars, ${ }^{93} \mathrm{Zr}$ had time to decay into ${ }^{93} \mathrm{Nb}$, since the $\beta$-decay half-life of ${ }^{93} \mathrm{Zr}$ is $1.53 \times 10^{6} \mathrm{yr}$.

These polluted stars thus appear as either barium stars, $\mathrm{CH}$ stars, or extrinsic $\mathrm{S}$ stars (depending on their $T_{\text {eff }}$ and metallicity), enriched in $\mathrm{Nb}$. Barium stars and extrinsic $\mathrm{S}$ stars are located along the diagonal in Fig. 14 (blue, black, and green symbols, respectively). The ratio $\mathrm{Zr} / \mathrm{Nb}$ is thus a powerful tool that can distinguish intrinsic from extrinsic stars (Bisterzo et al. 2010; Neyskens et al. 2015).

The barium stars in the present sample have been deliberately selected to be highly enriched in $s$-process elements: their maximum overabundance is around $[\mathrm{Zr} / \mathrm{Fe}] \sim 1.6$. Intrinsic $\mathrm{S}$ stars, in turn, show a lower maximum overabundance level (around $[\mathrm{Zr} / \mathrm{Fe}] \sim 0.9$ ). This is not surprising, since intrinsic $\mathrm{S}$ stars spend only a small portion of their TPAGB lifetime being classified as $\mathrm{S}$ stars. As they evolved along the AGB, third dredge-ups raise the surface $\mathrm{C} / \mathrm{O}$ ratio and rapidly turn the $\mathrm{S}$ star into a carbon star (with $\mathrm{C} / \mathrm{O}>1$ ). The latter are expected and known to be more $s$-process enriched indeed (see Extended Data Fig. 2 in Neyskens et al. 2015).

For the sake of clarity, we reproduce here the main predictions from Neyskens et al. (2015), based on the assumption of local equilibrium, i.e., the product $\left\langle\sigma_{i}\right\rangle N_{i}$ is constant along an isotopic chain (where $\left\langle\sigma_{i}\right\rangle$ and $N_{i}$ are the Maxwellian-averaged neutron-capture cross section and abundance of isotope $i$, respectively). The observed $([\mathrm{Zr} / \mathrm{Fe}],[\mathrm{Nb} / \mathrm{Fe}])$ ratios are then expected to fall on the relation

$\left[\frac{\mathrm{Zr}}{\mathrm{Fe}}\right]=\left[\frac{\mathrm{Nb}}{\mathrm{Fe}}\right]+\log \omega^{*}-\log \frac{N_{\odot}(\mathrm{Zr})}{N_{\odot}(\mathrm{Nb})}$,

where

$\omega^{*}=\left\langle\sigma_{93}\right\rangle\left[\frac{1}{\left\langle\sigma_{90}\right\rangle}+\frac{1}{\left\langle\sigma_{91}\right\rangle}+\frac{1}{\left\langle\sigma_{92}\right\rangle}+\frac{1}{\left\langle\sigma_{94}\right\rangle}\right]$.

The above relation further assumes that the neutron density is low enough for the branching at ${ }^{95} \mathrm{Zr}$ not to produce ${ }^{96} \mathrm{Zr}$. Thus, the $y$-intercept of the straight line of slope 1 defined by Eq. (2) in the $([\mathrm{Zr} / \mathrm{Fe}],[\mathrm{Nb} / \mathrm{Fe}])$ plane depends on the neutroncapture cross sections of the $\mathrm{Zr}$ isotopes, through the quantity $\omega^{*}$. Since these cross sections are temperature dependent, so is $\omega^{*}$, and hence the $y$-intercept of the diagonal. The location of the diagonal should thus give insights into the operation temperature of the $s$-process. To illustrate these principles, the band corresponding to $s$-process operating temperatures of 1 to $3 \times 10^{8} \mathrm{~K}$ is displayed in Fig. 14; this theoretical band assumes local nuclear equilibrium and is therefore independent of the metallicity (Eqs. (2) and (3)). The sensitivity of the method remains modest because the error bars on our data points prevent a precise determination of the temperature. Furthermore, detailed nucleosynthesis calculations in hot thermal pulses of AGB stars show that the assumption of local equilibrium is not necessarily valid. The situation is thus more complex than that described by Eqs. (2) and (3), as we discuss in Sect. 7.4.2.

\subsubsection{Predictions from STAREVOL}

The STAREVOL predictions for $[\mathrm{Fe} / \mathrm{H}]=-0.5$ (not assuming local equilibrium and not neglecting the ${ }^{95} \mathrm{Zr}-{ }^{96} \mathrm{Zr}$ branching) relative to $\mathrm{Nb}$ and $\mathrm{Zr}$ are displayed in Fig. 15 , where all ${ }^{93} \mathrm{Zr}$ is

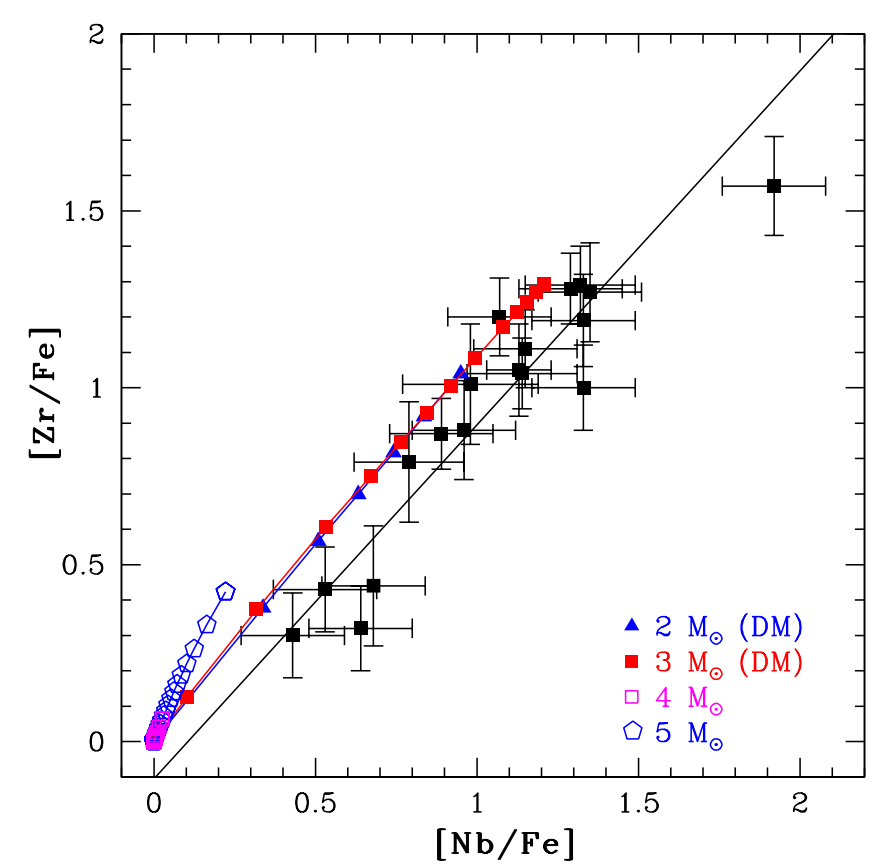

Fig. 15. Predictions from the STAREVOL code for extrinsic stars polluted by a $2,3,4$, or $5 M_{\odot}$ AGB star with $[\mathrm{Fe} / \mathrm{H}]=-0.5$. The $4 M_{\odot}$ predictions are superimposed on the $5 M_{\odot}$ values, but their track stops at $([\mathrm{Nb} / \mathrm{Fe}],[\mathrm{Zr} / \mathrm{Fe}]) \sim(0.03,0.07)$. For the 2 and $3 M_{\odot}$ models, diffusive mixing (DM) of protons in the ${ }^{12} \mathrm{C}$ pocket during the interpulse is modelled, whereas for the 4 and $5 M_{\odot}$ models, the $s$-process is assumed to be purely convective in the pulses. Each symbol corresponds to a thermal pulse enrichment. The black line corresponds to the fit to the barium stars (black squares).

assumed to have decayed into $\mathrm{Nb}$; therefore, only extrinsic stars are plotted.

The 2 and $3 M_{\odot}$ models follow a diagonal of slope 1 with a $\omega^{*}$ value of 15.8 , consistent with a $s$-process temperature of $10^{8} \mathrm{~K}$ typical of the operation of the ${ }^{13} \mathrm{C}(\alpha, \mathrm{n}){ }^{16} \mathrm{O}$ neutron source. However, for AGB stars more massive than $\sim 4 M_{\odot}$, the situation becomes more complex. First, given the greater envelope mass and the small pulse extent, many more third dredgeups are required to reach a given surface overabundance level, and at the end of the computations the enrichment remains modest. Second, it does not comply any longer with the picture drawn under the hypothesis of local equilibrium since the $\mathrm{Nb}$ and $\mathrm{Zr}$ abundances for these more massive stars (with hot pulses thus) fall above the predictions for the cooler pulses in lower-mass AGB stars. This result is in contradiction with the temperature dependence predicted by the local-equilibrium analysis (Fig. 14 and Sect. 7.4.1).

There are three reasons responsible for the breakdown of the local-equilibrium assumption. First, local equilibrium does not have enough time to establish since the duration of thermal pulses is short compared to the neutron-capture timescale for the $\mathrm{Zr}$ isotopes, especially in view of the rapidly varying profile of neutrons in the convective pulse and the small region of the thermal pulse subject to the neutron irradiation. Second, the neutron density in a pulse of the $4 M_{\odot}$ star (with $[\mathrm{Fe} / \mathrm{H}]=-0.5$ ) with base temperature of $3.7 \times 10^{8} \mathrm{~K}$ is high enough to bypass $\beta$-decay of ${ }^{95} \mathrm{Zr}$ and produce ${ }^{96} \mathrm{Zr}$. This results in values of $\mathrm{Zr} / \mathrm{Nb}$ as high as 27 , to be compared with $\mathrm{Zr} / \mathrm{Nb}$ in the range $12-17$ (or more exactly 15.8 , as found above for the 2 and $3 M_{\odot}$ stars) as predicted by the local equilibrium analysis (without ${ }^{96} \mathrm{Zr}$; see Fig. 1 of Neyskens et al. 2015). Third, with the high neutron densities 
found at the bottom of hot thermal pulses $\left(N_{\mathrm{n}} \approx 10^{12} \mathrm{~cm}^{-3}\right)$, an important amount of the unstable ${ }^{90} \mathrm{Sr}$ (with a half-life of $t_{1 / 2}=$ $28.8 \mathrm{y}),{ }^{90} \mathrm{Y}\left(t_{1 / 2}=2.7 \mathrm{~d}\right)$, and ${ }^{91} \mathrm{Y}\left(t_{1 / 2}=58.5 \mathrm{~d}\right)$ is produced. During the neutron irradiation, such long-lived branchings contribute to the production of ${ }^{91,92} \mathrm{Zr}$ isotopes but bypass ${ }^{90} \mathrm{Zr}$. After the neutron irradiation, their $\beta$-decays lead to a delayed increase in the $\mathrm{Zr}$ abundance without affecting the ${ }^{93} \mathrm{Zr}$ (thus ${ }^{93} \mathrm{Nb}$ ) yield, hence a higher value of the $\mathrm{Zr} / \mathrm{Nb}$ ratio.

All these reasons explain why the $([\mathrm{Zr} / \mathrm{Fe}],[\mathrm{Nb} / \mathrm{Fe}])$ curves corresponding to hot pulses in 4-5 $M_{\odot}$ AGB stars are located above the ${ }^{13} \mathrm{C}(\alpha, \mathrm{n}){ }^{16} \mathrm{O}$ locus in Fig. 15. Because of this behaviour, the discriminating power of the $([\mathrm{Zr} / \mathrm{Fe}],[\mathrm{Nb} / \mathrm{Fe}])$ diagram is restored, and in fact, turns out to be better than initially inferred from the simple local-equilibrium analysis (Fig. 14). Figure 15 clearly shows that all the extrinsic stars analysed so far are consistent with an $s$-process triggered by the ${ }^{13} \mathrm{C}(\alpha, \mathrm{n}){ }^{16} \mathrm{O}$ neutron source operating in 2-3 $M_{\odot}$ AGB stars (see, however, a caveat below) rather than in more massive ones, a conclusion in agreement with that reached in Sect. 7.1 on the basis of the $s$-process abundance pattern. This confirms conclusions from earlier studies (e.g. Busso et al. 2001) that the abundance pattern, as probed specifically by the [hs/ls] ratio, is compatible with the ${ }^{13} \mathrm{C}(\alpha, \mathrm{n}){ }^{16} \mathrm{O}$ neutron source.

It is noteworthy that no extrinsic stars seem to originate from more massive AGB progenitors. This could be the result of a combined effect of the lower probability of occurrence of more massive stars, and of the moderate levels of pollution expected from these stars (Fig. 15) due to large dilution factors. Therefore, extrinsic stars polluted by massive AGB stars, should they exist, would exhibit very modest enhancement levels, and might even not be detected as $s$-process-rich stars.

Many extrinsic stars in Fig. 15 are, however, off the STAREVOL $2-3 M_{\odot}$ predictions by a few tenths of a dex. Given the careful abundance calibration performed in the present paper, there are no obvious reasons why such a bias would affect our $\mathrm{Nb}$ abundances. However, as stressed in Sect. 4.2.3, the oscillator strengths for the two lines used to derive the $\mathrm{Zr}$ abundance have a tendency to yield abundances that are about 0.1 dex too low in the benchmark stars. This slight shift would be enough to reconcile observed $([\mathrm{Zr} / \mathrm{Fe}],[\mathrm{Nb} / \mathrm{Fe}])$ abundances with those predicted in 2-3 $M_{\odot}$ stars (Fig. 15). The uncertainty on the ${ }^{93} \mathrm{Zr}$ neutron-capture cross section (Tagliente et al. 2013) could also modify somewhat the STAREVOL predictions (see Fig. 1 of Neyskens et al. 2015, for an illustration of the impact of the cross-section uncertainties on $\omega^{*}$ ).

\subsubsection{Gaia parallaxes and determination of the mass of the extrinsic stars}

The positions of the stars in the HR diagram was derived using the procedure described by Escorza et al. (2017): the bolometric flux is computed by integrating the spectral energy distribution corresponding to a MARCS model atmosphere with the stellar parameters (effective temperatures, gravities, and metallicities) derived from the spectroscopic analysis (Table 1). Then the distance modulus corresponding to the TGAS parallax (The Tycho-Gaia astrometric solution; Michalik et al. 2015) available for 14 (out of 18) of our sample stars was computed to obtain the luminosity. Once the luminosity and effective temperature were known, the stellar mass was estimated by finding the STAREVOL evolutionary track of the corresponding metallicity that passes through this point in the HR diagram (Fig. 16). The resulting luminosities and masses are listed in
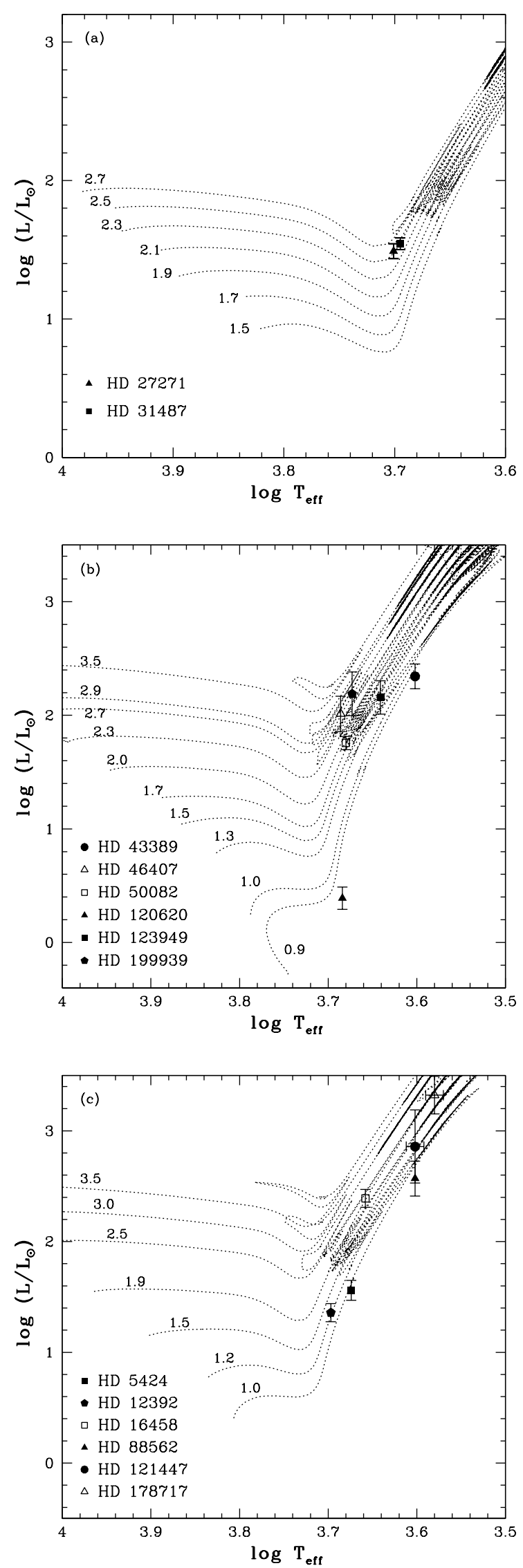

Fig. 16. HR diagram for the programme stars, ordered according to their metallicities: panel $a:[\mathrm{Fe} / \mathrm{H}] \approx 0.0$; panel $b:[\mathrm{Fe} / \mathrm{H}] \approx-0.25$; panel $c:[\mathrm{Fe} / \mathrm{H}] \approx-0.5$. The tracks are labelled according to the mass (in $M_{\odot}$ ). 
Table 5. Luminosities, masses, and metallicities for the programme stars.

\begin{tabular}{llllllll}
\hline \hline Name & {$[\mathrm{Fe} / \mathrm{H}]$} & $\log L_{\min }$ & $\begin{array}{l}\log L \\
\left(L_{\odot}\right)\end{array}$ & $\log L_{\max }$ & $M_{\min }$ & $\begin{array}{l}M \\
\left(M_{\odot}\right)\end{array}$ & $M_{\max }$ \\
\hline HD 5424 & -0.43 & 1.47 & 1.56 & 1.66 & 1.0 & 1.1 & 1.2 \\
HD 12392 & -0.38 & 1.28 & 1.36 & 1.45 & 1.0 & 1.1 & 1.2 \\
HD 16458 & -0.64 & 2.31 & 2.39 & 2.47 & 1.9 & 2.2 & 2.5 \\
HD 27271 & -0.07 & 1.44 & 1.49 & 1.55 & 2.3 & 2.5 & 2.7 \\
HD 31487 & -0.04 & 1.50 & 1.54 & 1.59 & 2.3 & 2.5 & 2.7 \\
HD 43389 & -0.35 & 2.23 & 2.34 & 2.47 & - & - & 0.9 \\
HD 46407 & -0.35 & 1.86 & 2.01 & 2.20 & 2.0 & 2.4 & 2.9 \\
HD 50082 & -0.32 & 1.70 & 1.76 & 1.82 & 1.5 & 1.7 & 2.0 \\
HD 88562 & -0.53 & 2.41 & 2.57 & 2.76 & 0.8 & 1.0 & 1.2 \\
HD 120620 & -0.29 & 0.29 & 0.39 & 0.50 & - & - & 0.9 \\
HD 121447 & -0.90 & 2.53 & 2.86 & 3.40 & 1.0 & 1.4 & 2.0 \\
HD 123949 & -0.23 & 2.01 & 2.16 & 2.34 & 1.0 & 1.3 & 1.7 \\
HD 178717 & -0.52 & 3.15 & 3.32 & 3.53 & 1.4 & 1.9 & 2.5 \\
HD 199939 & -0.22 & 2.00 & 2.19 & 2.44 & 2.0 & 2.7 & 3.5 \\
\hline
\end{tabular}

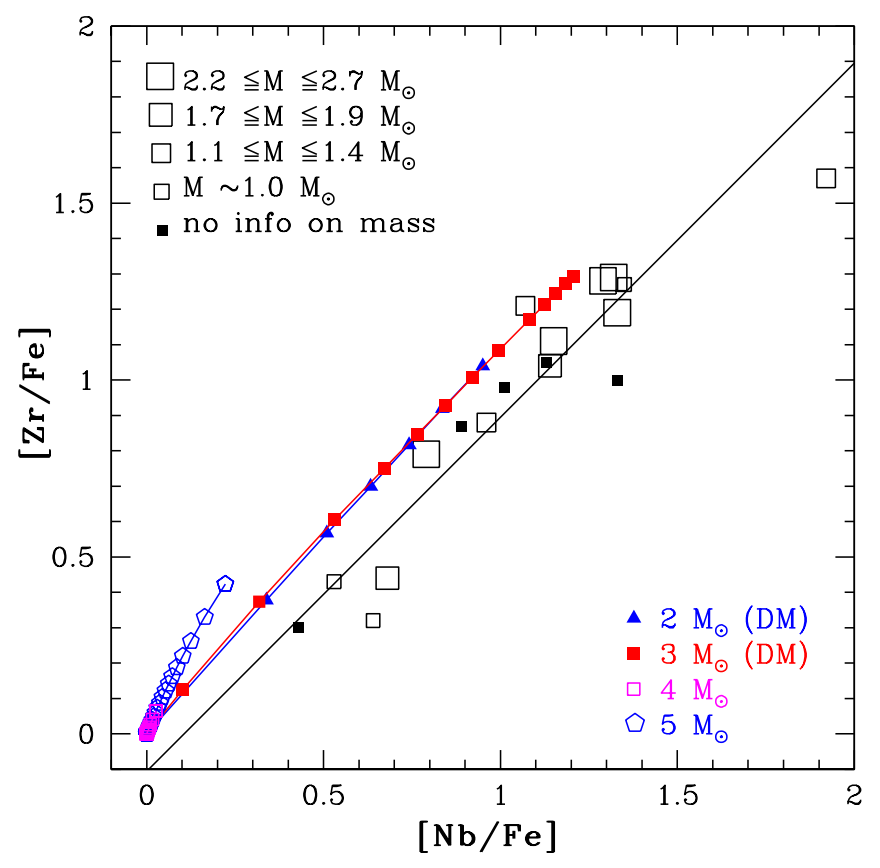

Fig. 17. Same as Fig. 15, but coded according to the mass of the extrinsic star whenever available.

Table 5, as are the uncertainties derived from the uncertainties on the TGAS parallaxes. Two objects (HD 43389 and HD 120620, see middle panel of Fig. 16) have luminosities fainter than the $0.9 M_{\odot}$ evolutionary track corresponding to their metallicity (though considering its error bar HD 43389 fits on the $0.9 M_{\odot}$ track). Only an upper limit for their mass is therefore listed in Table 5. We note that the position in the HR diagram of HD 120620 is consistent with its unusually high ${ }^{12} \mathrm{C} /{ }^{13} \mathrm{C}(\approx 90)$, indicative of a pre-first dredge-up composition.

The inferred masses of the barium stars range between 0.9 and $3 M_{\odot}$ and are compatible with the conclusions that the $s$ process material currently observed in the atmospheres of the extrinsic stars must have originated from a low-mass AGB companion. Because the $\mathrm{Zr} / \mathrm{Nb}$ ratio depends on the operation temperature of the $s$-process, thus on the mass of the AGB polluter, we might expect that the position of the barium star in the $([\mathrm{Zr} / \mathrm{Fe}],[\mathrm{Nb} / \mathrm{Fe}])$ plane depends on its mass as well. Figure 17 reveals no such correlation, however, perhaps because, as discussed above, stars polluted by more massive AGB stars never display overabundance levels as high as those in barium stars.

\subsection{Rubidium as a diagnostic for the operation of the ${ }^{22} \mathrm{Ne}$ neutron source}

Models predict higher $\mathrm{Rb}$ abundances in AGB stars where neutrons are produced by the ${ }^{22} \mathrm{Ne}(\alpha, \mathrm{n})^{25} \mathrm{Mg}$ reaction (Abia et al. 2001; van Raai et al. 2012). The amount of produced $\mathrm{Rb}$ depends on the ability of ${ }^{85} \mathrm{Kr}$ and ${ }^{86} \mathrm{Rb}$ to capture a neutron before decaying, since they act as branching points. The probability of this happening depends on the local neutron density (Beer \& Macklin 1989). The ${ }^{22} \mathrm{Ne}(\alpha, \mathrm{n})^{25} \mathrm{Mg}$ neutron source favours the production of ${ }^{87} \mathrm{Rb}$. Hence, the determination of the ${ }^{87} \mathrm{Rb} /{ }^{85} \mathrm{Rb}$ isotopic ratio in $\mathrm{AGB}$ stars could inform us about the neutron source but that ratio is impossible to measure in stellar spectra, even with a very high spectral resolution. Nevertheless, models predict that the ratio of $\mathrm{Rb}$ with other light $s$-process elements like $\mathrm{Sr}, \mathrm{Y}$, or $\mathrm{Zr}$ can be used for the same purpose. As shown in Fig. 18, derived $[\mathrm{Rb} / \mathrm{Zr}]$ ratios in AGB stars of different masses both in our Galaxy and the Magellanic Clouds vary from -1 to $1.2 \mathrm{dex}$ (Abia et al. 2001; García-Hernández et al. 2006; van Raai et al. 2012; Zamora et al. 2014; Perez-Mesa et al. 2017), the highest values (shaded region in Fig. 18) corresponding to the most massive AGB stars $\left(4-6 M_{\odot}\right)$, and negative values to lower mass AGB stars where ${ }^{13} \mathrm{C}(\alpha, \mathrm{n}){ }^{16} \mathrm{O}$ operates.

While HD 121447 shows a substantial $\mathrm{Rb}$ overabundance $([\mathrm{Rb} / \mathrm{Fe}]=0.63)$, this is a sequel to the very high overall $s$ process overabundances for this star. In terms of $[\mathrm{Rb} / \mathrm{Zr}](=$ -0.94), HD 121447 is not different from the other stars, which all have $[\mathrm{Rb} / \mathrm{Zr}]<0$ as expected when ${ }^{13} \mathrm{C}(\alpha, \mathrm{n}){ }^{16} \mathrm{O}$ operates (e.g. van Raai et al. 2012). In fact, none of the measured [Rb/Zr] values in our barium stars agrees with the $[\mathrm{Rb} / \mathrm{Zr}]$ values measured in intermediate-mass AGB stars (shaded region in Fig. 18) nor with the expectations for the operation of ${ }^{22} \mathrm{Ne}(\alpha, \mathrm{n})^{25} \mathrm{Mg}$ in thermal pulses occurring in more massive AGB stars (van Raai et al. 2012; Karakas \& Lugaro 2016).

\subsection{HD 100503: a CEMP-rs analogue at a relatively high metallicity $([\mathrm{Fe} / \mathrm{H}]=-0.7)$ ?}

Most of the N-rich stars show enrichments in $\mathrm{Na}$ (Fig. 5), Nb (Fig. 14), and exhibit high [hs/ls] ratios (Fig. 11 and top panel 


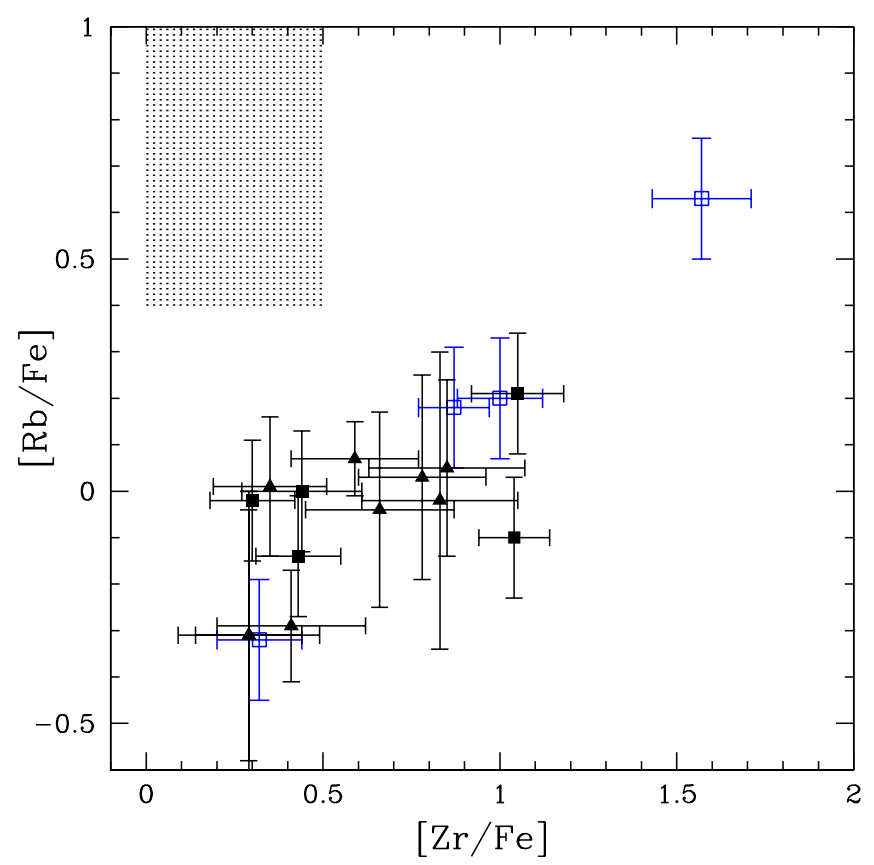

Fig. 18. Abundances of $\mathrm{Rb}$ vs. $\mathrm{Zr}$ for barium (squares) and extrinsic $\mathrm{S}$ stars (filled triangles). The four usual $\mathrm{N}$ - and Nb-rich barium stars are shown as open blue squares. Among these, HD 121447 is the most enriched in $\mathrm{Zr}$. The shaded area represents the observed range of $\mathrm{Rb}$ and $\mathrm{Zr}$ abundances in intermediate-mass AGB stars (van Raai et al. 2012), clearly incompatible with the range measured in barium stars.

Table 6. $[1 \mathrm{~s} / \mathrm{Fe}]$ and $[\mathrm{hs} / \mathrm{Fe}]$ in the programme stars.

\begin{tabular}{lcccc}
\hline \hline $\mathrm{HD}$ & {$[\mathrm{Fe} / \mathrm{H}]$} & {$[\mathrm{l} / \mathrm{Fe}]$} & {$[\mathrm{hs} / \mathrm{Fe}]$} & {$[\mathrm{hs} / \mathrm{ls}]$} \\
\hline 5424 & -0.43 & 1.11 & 1.75 & 0.64 \\
12392 & -0.38 & 1.11 & 1.41 & 0.30 \\
16458 & -0.64 & 1.24 & 1.37 & 0.13 \\
27271 & -0.07 & 0.79 & 0.65 & -0.14 \\
28159 & -0.50 & 0.29 & 0.67 & 0.38 \\
31487 & -0.04 & 1.11 & 1.50 & 0.39 \\
43389 & -0.35 & 0.57 & 1.38 & 0.81 \\
46407 & -0.35 & 1.22 & 1.53 & 0.31 \\
50082 & -0.32 & 0.96 & 1.39 & 0.43 \\
60197 & -0.60 & 0.89 & 0.97 & 0.08 \\
88562 & -0.53 & 0.64 & 1.09 & 0.45 \\
100503 & -0.72 & 1.09 & 1.71 & 0.62 \\
116869 & -0.44 & 0.86 & 1.12 & 0.26 \\
120620 & -0.29 & 1.33 & 1.56 & 0.23 \\
121447 & -0.90 & 1.48 & 2.31 & 0.83 \\
123949 & -0.23 & 0.90 & 1.25 & 0.35 \\
178717 & -0.52 & 0.66 & 0.80 & 0.26 \\
199939 & -0.22 & 1.27 & 1.70 & 0.43 \\
\hline
\end{tabular}

of Fig. 19 with data from Table 6). A further important property, not yet discussed, is their enrichment in the $r$-process elements $\mathrm{Sm}$ and Eu (bottom panel of Fig. 19). Figure 20 compares the abundances of $s$ and $r$ elements, in a way similar to that done for CEMP stars to distinguish CEMP- $s$ stars from CEMP- $r$ and CEMP-rs stars (Masseron et al. 2010). One of our target stars clearly stands out, namely HD 100503, which falls in the region occupied by CEMP-rs stars. This similarity suggests that a common nucleosynthetic process may be responsible for the chemical anomalies of CEMP- $r$ s stars and the barium star HD 100503.

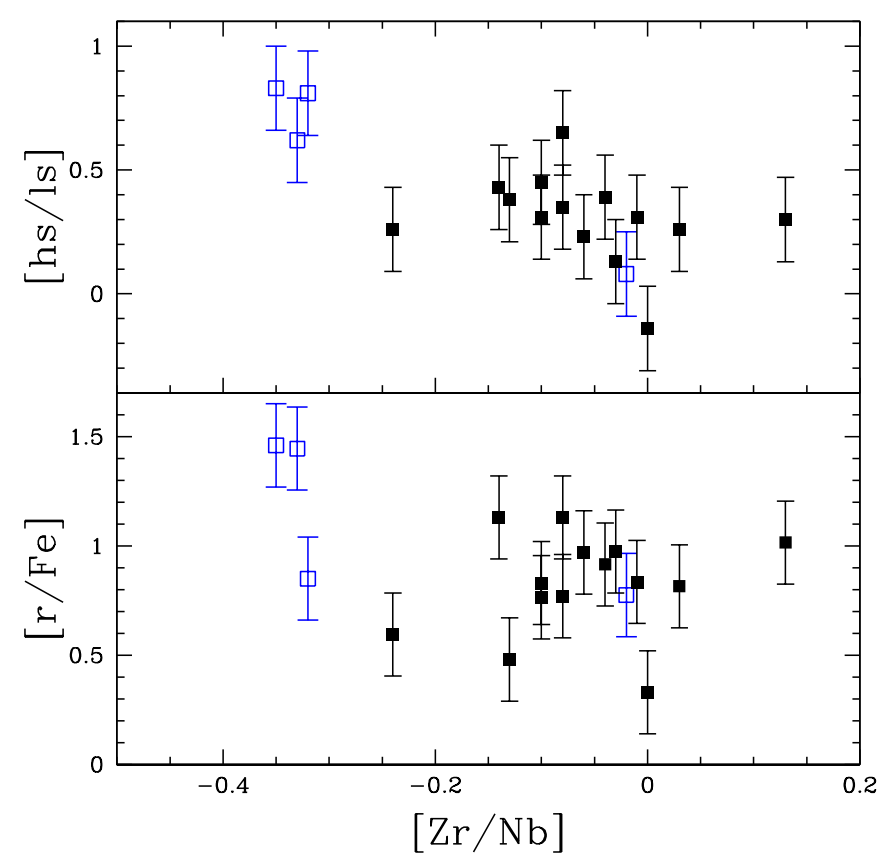

Fig. 19. Ratios of [hs/ls] (top panel, where $l s$ is the average of Sr, Y, and $\mathrm{Zr}$, and $h s$ the average of $\mathrm{La}$ and $\mathrm{Ce}$ ) and $[\mathrm{r} / \mathrm{Fe}]$ (bottom panel, where "r" stands for the average of the $[\mathrm{Sm} / \mathrm{Fe}]$ and $[\mathrm{Eu} / \mathrm{Fe}]$ ratios) are shown as a function of $[\mathrm{Zr} / \mathrm{Nb}]$. The four N-rich barium stars are shown as open blue squares, of which HD 100503 is found to be highly enriched in $r$-process elements.

We note that HD 121447 is characterised by a high [Eu/Fe] ratio, but it has an extremely high $[\mathrm{La} / \mathrm{Fe}]$ ratio, locating it on the $s$-process side in the $([\mathrm{La} / \mathrm{Fe}],[\mathrm{Eu} / \mathrm{Fe}])$ plane (Fig. 20). Therefore its connection with the CEMP-rs objects is less obvious.

The scenarios invoked to explain the $r$-process overabundances of CEMP-rs stars involve either an external pollution from a nearby supernova or the $i$-process (see references in Masseron et al. 2010), i.e. a process leading to neutron densities of the order of $N_{\mathrm{n}} \approx 10^{15} \mathrm{~cm}^{-3}$, intermediate between those of the $s$-process $\left(N_{\mathrm{n}} \approx 10^{8} \mathrm{~cm}^{-3}\right)$ and those of the $r$-process $\left(N_{\mathrm{n}} \geq 10^{20} \mathrm{~cm}^{-3}\right)$.

The operation of ${ }^{22} \mathrm{Ne}(\alpha, \mathrm{n})^{25} \mathrm{Mg}$ in the hot thermal pulses of intermediate-mass AGB stars (Goriely \& Siess 2005) leads to such intermediate neutron densities $\left(N_{\mathrm{n}} \approx 10^{11}-10^{14} \mathrm{~cm}^{-3}\right)$. Another possibility for the $i$-process operation would be the ingestion of protons in a He-burning convective region, as first described by Cowan \& Rose (1977). In this situation, protons are captured by ${ }^{12} \mathrm{C}$ and lead to the formation of ${ }^{13} \mathrm{C}$ which activates the ${ }^{13} \mathrm{C}(\alpha, \mathrm{n}){ }^{16} \mathrm{O}$ neutron source. The typical neutron densities in such conditions are of the order of $N_{\mathrm{n}} \approx 10^{15} \mathrm{~cm}^{-3}$, substantially higher than the $s$-process neutron densities when protons are mixed in the intershell region to form the ${ }^{13} \mathrm{C}$ pocket. Different sites have been proposed for this $i$-process, including (i) proton ingestion in very metal-poor low-mass stars during the core He-flash or at the beginning of the TP-AGB phase, (ii) the dredge-out in super-AGB stars, (iii) the late pulses in postAGB stars, and (iv) He-shell flashes in rapidly accreting white dwarfs (see Karakas \& Lattanzio 2014; Denissenkov et al. 2017, and references therein). We note that three-dimensional simulations show that turbulent entrainment of material could produce proton ingestion episodes at metallicities close to solar during the core He-flash (Mocák et al. 2008, 2009, 2010), and this could affect stars of masses $M \leq 2.25 M_{\odot}$. 


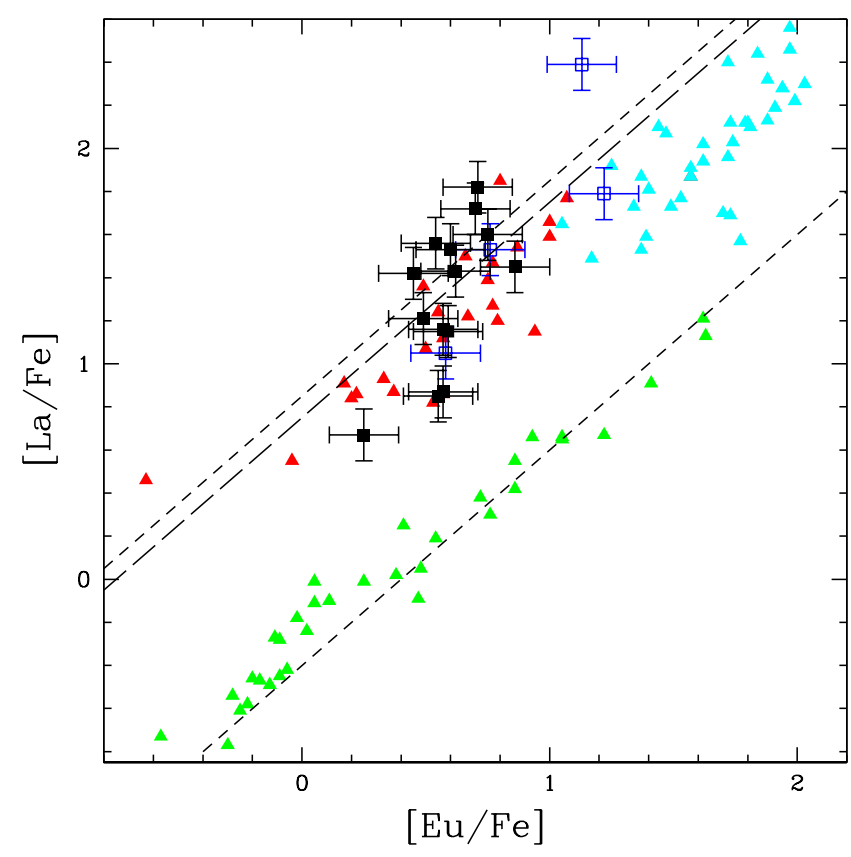

Fig. 20. Abundances of $[\mathrm{La} / \mathrm{Fe}]$ vs. $[\mathrm{Eu} / \mathrm{Fe}]$ in barium stars (squares) and in CEMP- $s$ (red triangles), CEMP- $r$ (green triangles), and CEMP$r s$ (cyan triangles) stars. Data for CEMP stars are from Masseron et al. (2010). The four usual N-rich barium stars are shown as open blue squares. The short-dashed line through the CEMP- $r$ stars corresponds to pure solar $r$-process (Goriely 1999), whereas the short-dashed line through the CEMP- $s$ stars corresponds to pure $s$-process nucleosynthesis predictions for a $0.8 M_{\odot}$ star (Masseron et al. 2006) and a $3 M_{\odot}$ (long-dashed line; Goriely \& Siess 2005) metal-poor AGB star.

The $i$-process has been recently invoked to explain not only CEMP-rs stars (e.g. Hampel et al. 2016), but also lowmetallicity post-AGB stars showing low lead and other heavy-s abundances that cannot be reconciled with standard $s$-process model predictions. More precisely, below $[\mathrm{Fe} / \mathrm{H}] \sim-0.7$, a very wide range of neutron irradiations is needed to explain the spread in heavy-s abundances observed in these objects (Lugaro et al. 2015; De Smedt et al. 2016; Hampel et al. 2016). These authors suggested that the $i$-process could meet this challenge. If HD 100503 turns out to be a genuine analogue of CEMP-rs stars, we note that it would be the first case found at such metallicity $([\mathrm{Fe} / \mathrm{H}]=-0.7)$.

\section{Conclusions}

Our analysis of the abundances of light $(\mathrm{C}, \mathrm{N}, \mathrm{Na}, \mathrm{Mg})$ and heavy elements in barium stars has shown that they are consistent with the operation of the ${ }^{13} \mathrm{C}(\alpha, \mathrm{n}){ }^{16} \mathrm{O}$ neutron source in $2-3 M_{\odot}$ AGB stars. We show in particular that the $\mathrm{Nb} / \mathrm{Zr}$ ratio offers a powerful diagnostic in that respect.

We have identified four N-rich stars with $[\mathrm{N} / \mathrm{Fe}]>0.7$ (HD 43389, HD 60197, HD 100503, and HD 121447). All of them, except HD 60197, have [Nb/Zr] and [hs/ls] ratios higher than any of the barium stars studied. Moreover, HD 100503 and HD 121447 are enriched in Eu, and the latter is also the most Narich star in our sample. The origin of these peculiarities remains a mystery; the high $\mathrm{N}$ abundance points towards a rather massive AGB companion undergoing hot-bottom burning, but this conclusion is at odds with the high [hs/ls] ratios that cannot be obtained from the activation of the ${ }^{22} \mathrm{Ne}(\alpha, \mathrm{n})^{25} \mathrm{Mg}$ source in those AGB stars. We conclude from the observed $s$-process abundance distributions that all the presently studied barium stars were polluted by low-mass $\left(M<4 M_{\odot}\right)$ AGB stars.

Of the four N-rich stars, HD 100503 has been identified as a possible analogue of CEMP-rs stars with the highest metallicity $([\mathrm{Fe} / \mathrm{H}]=-0.7)$ known so far.

Acknowledgements. D.K. acknowledges the support from Belgian Science Policy Office (BELSPO). This research has been partly funded by the Belgian Science Policy Office under contract BR/143/A2/STARLAB. S.V.E, T.M., and M.V.d.S. are thankful to "Fondation ULB" for its support. L.S. and S.G. are senior FRS-FNRS research associates. T.M. acknowledges support provided by the Spanish Ministry of Economy and Competitiveness (MINECO) under grant AYA-2017-88254-P. This research is based on observations performed with the Mercator telescope and the HERMES spectrograph. The Mercator telescope is operated thanks to grant number G.0C31.13 of the FWO under the "Big Science" initiative of the Flemish government. The HERMES spectrograph is supported by the Fund for Scientific Research of Flanders (FWO), the Research Council of K.U.Leuven, the Fonds National de la Recherche Scientifique (F.R.S.- FNRS), Belgium, the Royal Observatory of Belgium; the Observatoire de Genève, Switzerland; and the Thüringer Landessternwarte Tautenburg, Germany. This work has made use of data from the European Space Agency (ESA) mission Gaia (https://www.cosmos.esa.int/gaia), processed by the Gaia Data Processing and Analysis Consortium (DPAC, https://www.cosmos.esa.int/ web/gaia/dpac/consortium). Funding for the DPAC has been provided by national institutions, in particular the institutions participating in the Gaia Multilateral Agreement. The authors thank the referee for the very constructive comments.

\section{References}

Abia, C., Busso, M., Gallino, R., et al. 2001, ApJ, 559, 1117

Abia, C., Recio-Blanco, A., de Laverny, P., et al. 2009, ApJ, 694, 971

Abia, C., Cunha, K., Cristallo, S., et al. 2010, ApJ, 715, L94

Abia, C., Cunha, K., Cristallo, S., \& de Laverny, P. 2015, A\&A, 581, A88

Allen, D. M., \& Barbuy, B. 2006, A\&A, 454, 895

Alvarez, R., \& Plez, B. 1998, A\&A, 330, 1109

Alves-Brito, A., Karakas, A. I., Yong, D., Meléndez, J., \& Vásquez, S. 2011, A\&A, 536, A40

Amarsi, A. M., Asplund, M., Collet, R., \& Leenaarts, J. 2016, MNRAS, 455, 3735

Asplund, M., Grevesse, N., Sauval, A. J., Allende Prieto, C., \& Kiselman, D. 2005, A\&A, 435, 339

Asplund, M., Grevesse, N., Sauval, A. J., \& Scott, P. 2009, ARA\&A, 47, 481

Barbuy, B., Jorissen, A., Rossi, S. C. F., \& Arnould, M. 1992, A\&A, 262, 216

Beer, H., \& Macklin, R. L. 1989, ApJ, 339, 962

Bensby, T., \& Feltzing, S. 2006, MNRAS, 367, 1181

Bensby, T., Feltzing, S., Lundström, I., \& Ilyin, I. 2005, A\&A, 433, 185

Bidelman, W. P., \& Keenan, P. C. 1951, ApJ, 114, 473

Biémont, E., Grevesse, N., Hannaford, P., \& Lowe, R. M. 1981, ApJ, 248, 867

Bisterzo, S., Gallino, R., Straniero, O., Cristallo, S., \& Käppeler, F. 2010, MNRAS, 404, 1529

Bisterzo, S., Gallino, R., Käppeler, F., et al. 2015, MNRAS, 449, 506

Boothroyd, A. I., Sackmann, I.-J., \& Wasserburg, G. J. 1995, ApJ, 442, L21

Busso, M., Gallino, R., Lambert, D. L., Travaglio, C., \& Smith, V. V. 2001, ApJ, 557,802

Campbell, S. W., \& Lattanzio, J. C. 2008, A\&A, 490, 769

Corliss, C. H., \& Bozman, W. R. 1962, Experimental Transition Probabilities for Spectral Lines of Seventy Elements; derived NBS Tables of Spectral-Line Intensities

Cowan, J. J., \& Rose, W. K. 1977, ApJ, 212, 149

de Castro, D. B., Pereira, C. B., Roig, F., et al. 2016, MNRAS, 459, 4299

De Smedt, K., Van Winckel, H., Kamath, D., et al. 2016, A\&A, 587, A6

Denissenkov, P. A., Herwig, F., Battino, U., et al. 2017, ApJ, 834, L10

Doherty, C. L., Gil-Pons, P., Lau, H. H. B., Lattanzio, J. C., \& Siess, L. 2014a, MNRAS, 437, 195

Doherty, C. L., Gil-Pons, P., Lau, H. H. B., et al. 2014b, MNRAS, 441, 582

Eggleton, P. P., Dearborn, D. S. P., \& Lattanzio, J. C. 2008, ApJ, 677, 581

Escorza, A., Boffin, H. M. J., Jorissen, A., et al. 2017, A\&A, 608, A100

García-Hernández, D. A., García-Lario, P., Plez, B., et al. 2006, Science, 314, 1751

Goriely, S. 1999, A\&A, 342, 881

Goriely, S., \& Mowlavi, N. 2000, A\&A, 362, 599

Goriely, S., \& Siess, L. 2004, A\&A, 421, L25

Goriely, S., \& Siess, L. 2005, in From Lithium to Uranium: Elemental Tracers of Early Cosmic Evolution, eds. V. Hill, P. Francois, \& F. Primas, IAU Symp., 228,451 
Goriely, S., \& Siess, L. 2018, A\&A, 609, A29

Gorlova, N., Van Winckel, H., Vos, J., et al. 2013, in EAS Pub. Ser., eds. K. Pavlovski, A. Tkachenko, \& G. Torres, 64, 163

Gustafsson, B., Edvardsson, B., Eriksson, K., et al. 2008, A\&A, 486, 951

Hampel, M., Stancliffe, R. J., Lugaro, M., \& Meyer, B. S. 2016, ApJ, 831, 171

Harris, M. J., Lambert, D. L., \& Smith, V. V. 1985, ApJ, 292, 620

Heiter, U., Lind, K., Asplund, M., et al. 2015, Phys. Scr., 90, 054010

Hinkle, K., Wallace, L., Valenti, J., \& Harmer, D. 2000, Visible and Near Infrared Atlas of the Arcturus Spectrum 3727-9300 A (San Francisco: ASP)

Iben, Jr., I., \& Renzini, A. 1983, ARA\&A, 21, 271

Jofré, P., Heiter, U., Soubiran, C., et al. 2014, A\&A, 564, A133

Johnson, J. A. 2002, ApJS, 139, 219

Jorissen, A. 2004, in Asymptotic Giant Branch Stars, eds. H. J. Habing, \& H. Olofsson, A\&A Library (New York: Springer Verlag), 461

Jorissen, A., \& Mayor, M. 1988, A\&A, 198, 187

Jorissen, A., Smith, V. V., \& Lambert, D. L. 1992, A\&A, 261, 164

Jorissen, A., Frayer, D. T., Johnson, H. R., Mayor, M., \& Smith, V. V. 1993, A\&A, 271, 463

Jorissen, A., Hennen, O., Mayor, M., Bruch, A., \& Sterken, C. 1995, A\&A, 301, 707

Käppeler, F. 1999, Prog. Part. Nucl. Phys. (UK), 43, 419

Käppeler, F., Gallino, R., Bisterzo, S., \& Aoki, W. 2011, Rev. Mod. Phys., 83, 157

Karakas, A. I. \& Lattanzio, J. C. 2014, PASA, 31, e030

Karakas, A. I., \& Lugaro, M. 2016, ApJ, 825, 26

Keenan, P. C. 1942, ApJ, 96, 101

Lambert, D. L., \& Ries, L. M. 1981, ApJ, 248, 228

Lind, K., Asplund, M., Barklem, P. S., \& Belyaev, A. K. 2011, A\&A, 528, A103

Lugaro, M., Campbell, S. W., Van Winckel, H., et al. 2015, A\&A, 583, A77

Maeckle, R., Holweger, H., Griffin, R., \& Griffin, R. 1975, A\&A, 38, 239

Marigo, P. 2002, A\&A, 387, 507

Masseron, T., Van Eck, S., Famaey, B., et al. 2006, A\&A, 455, 1059

Masseron, T., Johnson, J. A., Plez, B., et al. 2010, A\&A, 509, A93

Masseron, T., Plez, B., Van Eck, S., et al. 2014, A\&A, 571, A47

Masseron, T., Merle, T., \& Hawkins, K. 2016, BACCHUS: Brussels Automatic Code for Characterizing High accUracy Spectra, Astrophysics Source Code Library [record ascl:1605.004]

Merle, T., Jorissen, A., Van Eck, S., Masseron, T., \& Van Winckel, H. 2016, A\&A, 586, A151

Michalik, D., Lindegren, L., \& Hobbs, D. 2015, A\&A, 574, A115

Mocák, M., Müller, E., Weiss, A., \& Kifonidis, K. 2008, A\&A, 490, 265

Mocák, M., Müller, E., Weiss, A., \& Kifonidis, K. 2009, A\&A, 501, 659

Mocák, M., Campbell, S. W., Müller, E., \& Kifonidis, K. 2010, A\&A, 520, A114
Neyskens, P., Van Eck, S., Jorissen, A., et al. 2015, Nature, 517, 174

Perez-Mesa, V., Zamora, O., Garcia-Hernandez, D. A., et al. 2017, A\&A 606, A20

Pilachowski, C. A., \& Pace, C. 2015, AJ, 150, 66

Plez, B. 2012, Turbospectrum: Code for Spectral Synthesis, Astrophysics Source Code Library [record ascl:1205.004]

Ramírez, I., \& Allende Prieto, C. 2011, ApJ, 743, 135

Raskin, G., van Winckel, H., Hensberge, H., et al. 2011, A\&A, 526, A69

Reddy, B. E., Tomkin, J., Lambert, D. L., \& Allende Prieto, C. 2003, MNRAS, 340, 304

Reddy, B. E., Lambert, D. L., \& Allende Prieto, C. 2006, MNRAS, 367, 1329

Reimers, D. 1975, Mem. Soc. Roy. Sci. Liège, 8, 369

Siess, L. 2006, A\&A, 448, 717

Siess, L., Dufour, E., \& Forestini, M. 2000, A\&A, 358, 593

Smiljanic, R., Porto de Mello, G. F., \& da Silva, L. 2007, A\&A, 468, 679

Smith, V. V. 1984, A\&A, 132, 326

Smith, V. V., \& Lambert, D. L. 1988, ApJ, 333, 219

Smith, V. V., \& Lambert, D. L. 1990, ApJS, 72, 387

Sneden, C., Pilachowski, C. A., \& Lambert, D. L. 1981, ApJ, 247, 1052

Sneden, C., Lucatello, S., Ram, R. S., Brooke, J. S. A., \& Bernath, P. 2014, ApJS, 214, 26

Suarez-Andres, L., Israelian, G., Gonzalez Hernandez, J. I., et al. 2017, A\&A, 599, A96

Tagliente, G., Milazzo, P. M., Fujii, K., et al. 2013, Phys. Rev. C, 87, 014622

Tomkin, J., \& Lambert, D. L. 1979, ApJ, 227, 209

Tout, C. A., \& Eggleton, P. P. 1988, MNRAS, 231, 823

Van der Swaelmen, M., Hill, V., Primas, F., \& Cole, A. A. 2013, A\&A, 560, A44

Van der Swaelmen, M., Boffin, H. M. J., Jorissen, A., \& Van Eck, S. 2017, A\&A, 597, A68

Van Eck, S., \& Jorissen, A. 1999, A\&A, 345, 127

Van Eck, S., \& Jorissen, A. 2000, A\&A, 360, 196

Van Eck, S., Jorissen, A., Udry, S., et al. 2000, A\&AS, 145, 51

Van Eck, S., Neyskens, P., Jorissen, A., et al. 2017, A\&A, 601, A10

van Raai, M. A., Lugaro, M., Karakas, A. I., García-Hernández, D. A., \& Yong, D. 2012, A\&A, 540, A44

Vanture, A. D., Wallerstein, G., Gallino, R., \& Masera, S. 2003, ApJ, 587, 384

Vassiliadis, E., \& Wood, P. R. 1993, ApJ, 413, 641

Wilson, R. E. 1953, General Catalogue of Stellar Radial Velocities (Washington, DC: Carnegie Institute Washington D.C. Publication)

Worley, C. C., Cottrell, P. L., Freeman, K. C., \& Wylie-de Boer, E. C. 2009, MNRAS, 400, 1039

Zamora, O., García-Hernández, D. A., Plez, B., \& Manchado, A. 2014, A\&A, 564, L4 


\section{Appendix $\mathrm{A}: \chi^{2}$ fitting regions}

Table A.1. Boundaries (expressed in $\mathrm{nm}$ ) of the continuum (C) and band (B) spectral regions used in the $\chi^{2}$ fitting (Sect. 3).

\begin{tabular}{llll}
\hline \hline$\lambda_{B, i}$ & $\lambda_{B, f}$ & $\lambda_{C, i}$ & $\lambda_{C, f}$ \\
\hline 400.0 & 420.0 & 408.0 & 412.0 \\
420.0 & 440.0 & 432.0 & 434.0 \\
440.0 & 458.0 & 456.0 & 458.0 \\
458.0 & 480.0 & 472.0 & 476.0 \\
480.0 & 490.0 & 489.4 & 489.9 \\
490.0 & 495.0 & 494.0 & 495.0 \\
495.0 & 516.0 & 515.5 & 516.0 \\
516.0 & 530.0 & 516.0 & 516.3 \\
530.0 & 544.0 & 543.0 & 544.5 \\
544.0 & 565.0 & 560.5 & 562.0 \\
565.0 & 575.5 & 573.0 & 573.7 \\
575.5 & 588.0 & 580.0 & 582.0 \\
588.0 & 615.0 & 613.0 & 615.0 \\
615.0 & 647.0 & 645.0 & 647.0 \\
647.0 & 671.5 & 670.0 & 671.5 \\
671.5 & 685.0 & 683.0 & 685.0 \\
703.0 & 720.0 & 703.0 & 705.0 \\
\hline
\end{tabular}

\section{Appendix B: Line list}

Table B.1 presents the lines used in the present abundance analysis.

Table B.1. Lines used in the abundance analysis.

\begin{tabular}{|c|c|c|}
\hline $\begin{array}{l}\lambda \\
(\AA)\end{array}$ & $\begin{array}{l}\chi_{\text {low }} \\
(\mathrm{eV})\end{array}$ & $\log g f$ \\
\hline $\begin{array}{l}\text { C I } \\
5380.337 \\
5553.174\end{array}$ & $\begin{array}{l}7.685 \\
8.643\end{array}$ & $\begin{array}{l}-1.615 \\
-2.370\end{array}$ \\
\hline $\begin{array}{l}\text { O I } \\
6300.304 \\
6363.776 \\
7771.941 \\
7774.161 \\
7775.338\end{array}$ & $\begin{array}{l}0.000 \\
0.020 \\
9.146 \\
9.146 \\
9.146\end{array}$ & $\begin{array}{r}-9.715 \\
-10.190 \\
0.369 \\
0.223 \\
0.001 \\
\end{array}$ \\
\hline $\begin{array}{l}\mathrm{NaI} \\
6154.226 \\
6160.747\end{array}$ & $\begin{array}{l}2.102 \\
2.104 \\
\end{array}$ & $\begin{array}{l}-1.547 \\
-1.246 \\
\end{array}$ \\
\hline $\begin{array}{l}\text { Mg I } \\
6318.717 \\
6319.237 \\
8717.810 \\
8736.006 \\
8736.019 \\
8736.019 \\
8736.029 \\
\end{array}$ & $\begin{array}{l}5.108 \\
5.108 \\
5.933 \\
5.946 \\
5.946 \\
5.946 \\
5.946 \\
\end{array}$ & $\begin{array}{l}-2.103 \\
-2.324 \\
-1.850 \\
-1.930 \\
-0.690 \\
-1.970 \\
-1.020 \\
\end{array}$ \\
\hline $\begin{array}{l}\mathrm{Rb} \mathrm{I} \\
7800.259 \\
7947.597\end{array}$ & $\begin{array}{l}0.000 \\
0.000 \\
\end{array}$ & $\begin{array}{r}0.137 \\
-0.167 \\
\end{array}$ \\
\hline $\begin{array}{l}\text { Sr I } \\
4962.259 \\
6408.459 \\
7070.070 \\
\text { Sr II } \\
4872.494\end{array}$ & $\begin{array}{l}1.847 \\
2.271 \\
1.847 \\
\\
1.798\end{array}$ & $\begin{array}{r}0.200 \\
0.510 \\
-0.030 \\
-0.060\end{array}$ \\
\hline $\begin{array}{l}\text { Y I } \\
6435.004 \\
5630.134 \\
\text { Y II } \\
4883.684 \\
4900.124 \\
5087.416 \\
5200.406 \\
5289.815 \\
5320.782 \\
5402.774 \\
5544.611 \\
5728.890 \\
6795.414 \\
7881.880\end{array}$ & $\begin{array}{l}1.084 \\
1.033 \\
1.084 \\
0.992 \\
1.033 \\
1.084 \\
1.839 \\
1.738 \\
1.839 \\
1.738 \\
1.840\end{array}$ & $\begin{array}{r}0.265 \\
0.103 \\
-0.170 \\
-0.570 \\
-1.850 \\
-1.950 \\
-0.630 \\
-1.090 \\
-1.120 \\
-1.030 \\
-0.570\end{array}$ \\
\hline $\begin{array}{l}\text { Zr I } \\
7819.374 \\
7849.374 \\
\text { Zr II } \\
4379.742 \\
5112.270 \\
5350.089 \\
5350.350 \\
\end{array}$ & $\begin{array}{l}1.532 \\
1.665 \\
1.827 \\
1.773 \\
\end{array}$ & $\begin{array}{l}-0.380 \\
-1.300 \\
-0.356 \\
-0.850 \\
-1.240 \\
-1.160 \\
\end{array}$ \\
\hline
\end{tabular}


Table B.1. continued.

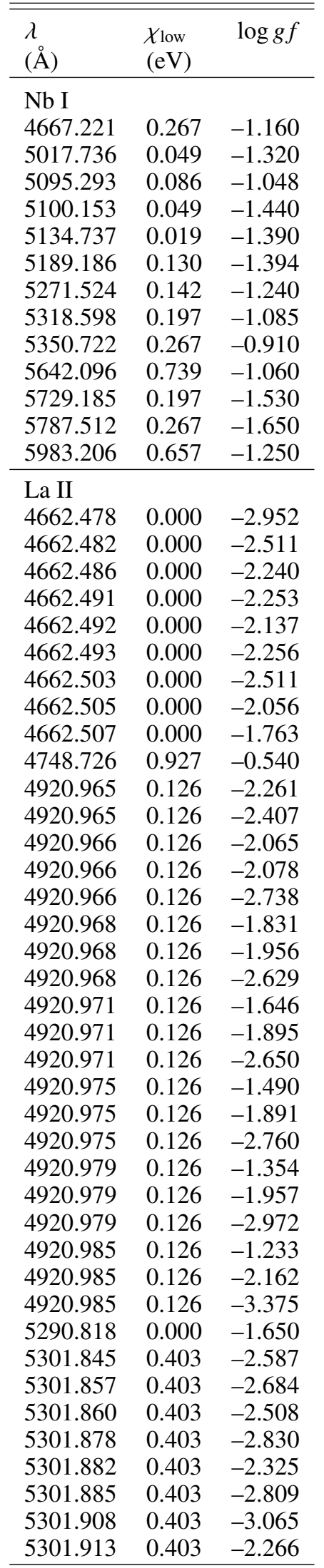

Table B.1. continued.

\begin{tabular}{lll}
\hline \hline$\lambda$ & $\chi$ low & $\log g f$ \\
$(\AA)$ & $(\mathrm{eV})$ & \\
\hline 5301.917 & 0.403 & -2.391 \\
5301.946 & 0.403 & -3.483 \\
5301.953 & 0.403 & -2.300 \\
5301.958 & 0.403 & -2.120 \\
5302.001 & 0.403 & -2.483 \\
5302.008 & 0.403 & -1.913 \\
5302.067 & 0.403 & -1.742 \\
5302.582 & 2.058 & -0.435 \\
5303.513 & 0.321 & -1.874 \\
5303.513 & 0.321 & -2.363 \\
5303.514 & 0.321 & -3.062 \\
5303.531 & 0.321 & -2.167 \\
5303.532 & 0.321 & -2.247 \\
5303.532 & 0.321 & -2.622 \\
5303.546 & 0.321 & -2.366 \\
5303.546 & 0.321 & -2.622 \\
5303.547 & 0.321 & -2.351 \\
6172.721 & 0.126 & -2.253 \\
6262.113 & 0.403 & -3.047 \\
6262.114 & 0.403 & -2.901 \\
6262.132 & 0.403 & -2.705 \\
6262.134 & 0.403 & -2.718 \\
6262.135 & 0.403 & -3.378 \\
6262.164 & 0.403 & -2.471 \\
6262.166 & 0.403 & -2.596 \\
6262.169 & 0.403 & -3.269 \\
6262.208 & 0.403 & -2.286 \\
6262.212 & 0.403 & -2.535 \\
6262.215 & 0.403 & -3.290 \\
6262.266 & 0.403 & -2.130 \\
6262.271 & 0.403 & -2.531 \\
6262.275 & 0.403 & -3.400 \\
6262.338 & 0.403 & -1.994 \\
6262.343 & 0.403 & -2.597 \\
6262.348 & 0.403 & -3.612 \\
6262.422 & 0.403 & -1.873 \\
6262.429 & 0.403 & -2.802 \\
6262.434 & 0.403 & -4.015 \\
6390.455 & 0.321 & -2.012 \\
6390.468 & 0.321 & -2.183 \\
6390.468 & 0.321 & -2.752 \\
6390.479 & 0.321 & -2.570 \\
6390.479 & 0.321 & -3.752 \\
6390.480 & 0.321 & -2.390 \\
6390.489 & 0.321 & -2.536 \\
6390.489 & 0.321 & -3.334 \\
6390.490 & 0.321 & -2.661 \\
6390.496 & 0.321 & -3.100 \\
6390.497 & 0.321 & -2.595 \\
6390.590 .506 & 0.321 & -3.079 \\
\hline & 0.321 & -2.954 \\
6.321 & -2.778 \\
631 & -2.857 \\
\hline & &
\end{tabular}


Table B.1. continued.

\begin{tabular}{|c|c|c|}
\hline $\begin{array}{l}\lambda \\
(\AA)\end{array}$ & $\begin{array}{l}\chi_{\text {low }} \\
(\mathrm{eV})\end{array}$ & $\log g f$ \\
\hline $\begin{array}{l}\text { Ce II } \\
4515.848 \\
4562.359 \\
4628.169 \\
4943.441 \\
5274.229 \\
5330.556 \\
5472.279 \\
5975.818 \\
6043.373 \\
6051.815\end{array}$ & $\begin{array}{l}1.058 \\
0.478 \\
0.516 \\
1.206 \\
1.044 \\
0.869 \\
1.247 \\
1.327 \\
1.206 \\
3.486\end{array}$ & $\begin{array}{r}-0.240 \\
0.230 \\
0.200 \\
-0.360 \\
0.130 \\
-0.400 \\
-0.100 \\
-0.460 \\
-0.480 \\
-0.210\end{array}$ \\
\hline $\begin{array}{l}\text { Nd II } \\
4797.150 \\
4947.020 \\
4961.387 \\
5089.832 \\
5092.788 \\
5132.328 \\
5212.360 \\
5276.869 \\
5293.160 \\
5311.450 \\
5319.810 \\
5356.967 \\
5361.165 \\
5361.467\end{array}$ & $\begin{array}{l}0.559 \\
0.559 \\
0.631 \\
0.205 \\
0.380 \\
0.559 \\
0.205 \\
0.859 \\
0.823 \\
0.986 \\
0.550 \\
1.264 \\
0.559 \\
0.680\end{array}$ & $\begin{array}{r}-0.690 \\
-1.130 \\
-0.710 \\
-1.160 \\
-0.610 \\
-0.710 \\
-0.960 \\
-0.440 \\
0.100 \\
-0.420 \\
-0.140 \\
-0.280 \\
-1.480 \\
-0.370\end{array}$ \\
\hline $\begin{array}{l}\text { Pr II } \\
5219.045 \\
5220.108 \\
5259.728 \\
5322.772\end{array}$ & $\begin{array}{l}0.795 \\
0.796 \\
0.633 \\
0.483 \\
\end{array}$ & $\begin{array}{r}-0.053 \\
0.298 \\
0.114 \\
-0.141 \\
\end{array}$ \\
\hline $\begin{array}{l}\text { Sm II } \\
4318.926 \\
4499.475 \\
4519.630 \\
4566.200 \\
4577.688 \\
4615.440 \\
4726.026 \\
\end{array}$ & $\begin{array}{l}0.277 \\
0.248 \\
0.544 \\
0.333 \\
0.248 \\
0.544 \\
0.333 \\
\end{array}$ & $\begin{array}{l}-0.250 \\
-0.870 \\
-0.350 \\
-0.590 \\
-0.650 \\
-0.690 \\
-1.250 \\
\end{array}$ \\
\hline $\begin{array}{l}\text { Eu II } \\
6437.601 \\
6437.603 \\
6437.606 \\
6437.609 \\
6437.617 \\
6437.619 \\
6437.620 \\
6437.623 \\
6437.627 \\
6437.627 \\
6437.630 \\
6437.633 \\
6437.633 \\
\end{array}$ & $\begin{array}{l}1.320 \\
1.320 \\
1.320 \\
1.320 \\
1.320 \\
1.320 \\
1.320 \\
1.320 \\
1.320 \\
1.320 \\
1.320 \\
1.320 \\
1.320 \\
\end{array}$ & $\begin{array}{l}-0.960 \\
-0.960 \\
-2.191 \\
-2.191 \\
-2.191 \\
-2.191 \\
-1.070 \\
-1.998 \\
-1.070 \\
-1.998 \\
-1.181 \\
-1.956 \\
-1.956\end{array}$ \\
\hline
\end{tabular}

Table B.1. continued.

\begin{tabular}{lll}
\hline \hline$\lambda$ & $\chi_{\text {low }}$ & $\log g f$ \\
$(\AA)$ & $(\mathrm{eV})$ & \\
\hline 6437.633 & 1.320 & -1.998 \\
6437.635 & 1.320 & -1.287 \\
6437.635 & 1.320 & -2.010 \\
6437.635 & 1.320 & -2.206 \\
6437.637 & 1.320 & -1.377 \\
6437.637 & 1.320 & -1.428 \\
6437.637 & 1.320 & -2.010 \\
6437.639 & 1.320 & -2.206 \\
6437.640 & 1.320 & -1.998 \\
6437.647 & 1.320 & -1.181 \\
6437.652 & 1.320 & -1.956 \\
6437.657 & 1.320 & -1.956 \\
6437.662 & 1.320 & -1.287 \\
6437.667 & 1.320 & -2.010 \\
6437.669 & 1.320 & -2.010 \\
6437.674 & 1.320 & -1.377 \\
6437.677 & 1.320 & -2.206 \\
6437.679 & 1.320 & -2.206 \\
6437.682 & 1.320 & -1.428 \\
6645.055 & 1.380 & -1.823 \\
6645.057 & 1.380 & -0.516 \\
6645.058 & 1.380 & -3.466 \\
6645.061 & 1.380 & -0.516 \\
6645.067 & 1.380 & -1.823 \\
6645.070 & 1.380 & -0.592 \\
6645.073 & 1.380 & -1.628 \\
6645.075 & 1.380 & -3.466 \\
6645.077 & 1.380 & -3.149 \\
6645.080 & 1.380 & -0.672 \\
6645.085 & 1.380 & -1.583 \\
6645.086 & 1.380 & -0.592 \\
6645.087 & 1.380 & -0.754 \\
6645.091 & 1.380 & -3.076 \\
6645.093 & 1.380 & -0.838 \\
6645.093 & 1.380 & -1.634 \\
6645.094 & 1.380 & -1.628 \\
6645.097 & 1.380 & -0.921 \\
6645.099 & 1.380 & -1.829 \\
6645.100 & 1.380 & -3.244 \\
6645.101 & 1.380 & -3.149 \\
6645.108 & 1.380 & -0.672 \\
6645.116 & 1.380 & -1.583 \\
6645.123 & 1.380 & -3.076 \\
6645.127 & 1.380 & -0.754 \\
6645.134 & 1.380 & -1.634 \\
6645.140 & 1.380 & -3.244 \\
6645.141 & 1.380 & -0.838 \\
6645.148 & 1.380 & -1.829 \\
6645.153 & 1.380 & -0.921 \\
\hline & &
\end{tabular}




\section{Appendix C: Elemental abundances for the programme stars}

Table C.1. Elemental abundances.

\begin{tabular}{|c|c|c|c|c|c|c|c|c|c|c|}
\hline & \multirow[b]{2}{*}{$Z$} & \multirow[b]{2}{*}{$\log _{\odot} \epsilon^{a}$} & \multicolumn{4}{|c|}{ HD 5424} & \multicolumn{4}{|c|}{ HD 12392} \\
\hline & & & $\log \epsilon$ & $\sigma_{s}(\mathrm{~N})$ & {$[\mathrm{X} / \mathrm{H}]$} & {$[\mathrm{X} / \mathrm{Fe}] \pm \sigma_{[X / F e]}$} & $\log \epsilon$ & $\sigma_{s}(\mathrm{~N})$ & {$[\mathrm{X} / \mathrm{H}]$} & {$[\mathrm{X} / \mathrm{Fe}] \pm \sigma_{[X / F e]}$} \\
\hline $\begin{array}{l}\mathrm{C} \\
{ }^{12} \mathrm{C} /{ }^{13} \mathrm{C}\end{array}$ & 6 & 8.43 & $\begin{array}{c}8.30 \\
19\end{array}$ & $0.08(8)$ & -0.13 & $0.30 \pm 0.12$ & $\begin{array}{c}8.50 \\
19\end{array}$ & $0.05(10)$ & 0.07 & $0.45 \pm 0.12$ \\
\hline $\mathrm{N}$ & 7 & 7.83 & 8.07 & $0.07(55)$ & 0.24 & $0.67 \pm 0.20$ & 8.00 & $0.08(50)$ & 0.17 & $0.55 \pm 0.21$ \\
\hline $\mathrm{O}$ & 8 & 8.69 & 8.60 & $-(1)$ & -0.09 & $0.34 \pm 0.18$ & 8.70 & $-(2)$ & 0.01 & $0.38 \pm 0.18$ \\
\hline $\mathrm{Na} \mathrm{I}$ & 11 & 6.24 & 5.93 & $0.04(2)$ & -0.31 & $0.12 \pm 0.13$ & 6.00 & $0.05(3)$ & -0.24 & $0.14 \pm 0.16$ \\
\hline $\mathrm{Mg} \mathrm{I}$ & 12 & 7.60 & - & - & - & - & 7.50 & $0.17(3)$ & -0.10 & $0.26 \pm 0.24$ \\
\hline $\mathrm{Fe}$ & 26 & 7.50 & 7.07 & $0.19(43)$ & -0.43 & - & 7.12 & $0.14(44)$ & -0.38 & - \\
\hline $\mathrm{Rb} \mathrm{I}$ & 37 & 2.52 & 2.3: & - & -0.22 & $0.21 \pm 0.14$ & - & - & - & - \\
\hline Sr I & 38 & 2.87 & 3.35 & $0.07(2)$ & 0.48 & $0.91 \pm 0.11$ & 3.42 & $0.03(2)$ & 0.55 & $0.93 \pm 0.11$ \\
\hline $\mathrm{Sr}$ II & 38 & 2.87 & 3.42 & $0.15(3)$ & 0.55 & $0.98 \pm 0.15$ & 3.55 & $0.05(3)$ & 0.68 & $1.06 \pm 0.12$ \\
\hline Y I & 39 & 2.21 & 2.58 & $0.14(4)$ & 0.37 & $0.80 \pm 0.17$ & 2.73 & $0.05(4)$ & 0.52 & $0.90 \pm 0.16$ \\
\hline Y II & 39 & 2.21 & 3.08 & $0.13(6)$ & 0.87 & $1.30 \pm 0.11$ & 2.91 & $0.13(6)$ & 0.70 & $1.08 \pm 0.13$ \\
\hline $\mathrm{Zr} \mathrm{I}$ & 40 & 2.58 & 3.20 & $0.06(2)$ & 0.62 & $1.05 \pm 0.13$ & 3.40 & $0.05(2)$ & 0.82 & $1.20 \pm 0.11$ \\
\hline $\mathrm{Zr}$ II & 40 & 2.58 & 3.78 & $0.15(3)$ & 1.20 & $1.63 \pm 0.17$ & 3.55 & $0.15(2)$ & 0.97 & $1.35 \pm 0.14$ \\
\hline $\mathrm{Nb} \mathrm{I}$ & 41 & 1.46 & 2.16 & $0.15(4)$ & 0.70 & $1.13 \pm 0.10$ & 2.15 & $0.02(1)$ & 0.69 & $1.07 \pm 0.16$ \\
\hline Ba II & 56 & 2.18 & 3.90: & - & 1.72 & $2.15 \pm 0.14$ & 3.3: & - & 1.12 & $1.50 \pm 0.10$ \\
\hline La II & 57 & 1.10 & 2.49 & $0.18(5)$ & 1.39 & $1.82 \pm 0.11$ & 2.17 & $0.09(8)$ & 1.07 & $1.45 \pm 0.12$ \\
\hline Ce II & 58 & 1.58 & 2.82 & $0.11(7)$ & 1.24 & $1.67 \pm 0.13$ & 2.56 & $0.07(7)$ & 0.98 & $1.36 \pm 0.11$ \\
\hline Pr II & 59 & 0.72 & 2.05 & $0.16(4)$ & 1.33 & $1.76 \pm 0.10$ & 1.70 & $0.06(4)$ & 0.98 & $1.36 \pm 0.10$ \\
\hline Nd II & 60 & 1.42 & 2.70 & $0.11(10)$ & 1.28 & $1.71 \pm 0.10$ & 2.44 & $0.09(11)$ & 1.02 & $1.40 \pm 0.10$ \\
\hline Sm II & 62 & 0.96 & 2.08 & $0.14(3)$ & 1.12 & $1.55 \pm 0.15$ & 1.75 & $0.07(2)$ & 0.79 & $1.17 \pm 0.13$ \\
\hline \multirow[t]{3}{*}{ Eu II } & 63 & 0.52 & 0.80 & $0.10(2)$ & 0.28 & $0.71 \pm 0.15$ & 1.00 & $0.03(4)$ & 0.48 & $0.86 \pm 0.13$ \\
\hline & & & \multicolumn{4}{|c|}{ HD 16458} & \multicolumn{4}{|c|}{ HD 27271} \\
\hline & $Z$ & $\log _{\odot} \epsilon^{a}$ & $\log \epsilon$ & $\sigma_{s}(\mathrm{~N})$ & {$[\mathrm{X} / \mathrm{H}]$} & {$[\mathrm{X} / \mathrm{Fe}] \pm \sigma_{[X / F e]}$} & $\log \epsilon$ & $\sigma_{s}(\mathrm{~N})$ & {$[\mathrm{X} / \mathrm{H}]$} & {$[\mathrm{X} / \mathrm{Fe}] \pm \sigma_{[X / F e]}$} \\
\hline $\begin{array}{l}\mathrm{C} \\
{ }^{12} \mathrm{C} /{ }^{13} \mathrm{C}\end{array}$ & 6 & 8.43 & $\begin{array}{c}8.07 \\
11\end{array}$ & $0.05(9)$ & -0.36 & $0.28 \pm 0.12$ & $\begin{array}{c}8.30 \\
12\end{array}$ & $0.06(5)$ & -0.13 & $-0.06 \pm 0.08$ \\
\hline $\mathrm{N}$ & 7 & 7.83 & 7.58 & $0.05(61)$ & -0.25 & $0.39 \pm 0.20$ & 8.30 & $0.03(50)$ & 0.47 & $0.54 \pm 0.21$ \\
\hline $\mathrm{O}$ & 8 & 8.69 & 8.10 & $0.14(2)$ & -0.59 & $0.05 \pm 0.18$ & 8.70 & $-(2)$ & 0.01 & $0.08 \pm 0.17$ \\
\hline $\mathrm{Na} \mathrm{I}$ & 11 & 6.24 & 6.30 & $0.14(2)$ & 0.06 & $0.70 \pm 0.15$ & 6.60 & $0.17(3)$ & 0.26 & $0.33 \pm 0.15$ \\
\hline $\mathrm{Mg} \mathrm{I}$ & 12 & 7.60 & - & - & - & - & 7.60 & $-(1)$ & -0.06 & $0.01 \pm 0.26$ \\
\hline $\mathrm{Fe}$ & 26 & 7.50 & 6.86 & $0.15(35)$ & -0.64 & - & 7.43 & $0.16(44)$ & -0.07 & - \\
\hline $\mathrm{Rb} \mathrm{I}$ & 37 & 2.52 & - & - & - & - & 1.7: & - & -0.82 & $-0.75 \pm 0.15$ \\
\hline Sr I & 38 & 2.87 & 3.48 & $0.18(2)$ & 0.61 & $1.25 \pm 0.16$ & 3.45 & $0.10(2)$ & 0.58 & $0.65 \pm 0.12$ \\
\hline $\mathrm{Sr}$ II & 38 & 2.87 & 3.60 & $0.17(3)$ & 0.73 & $1.37 \pm 0.16$ & 3.60 & $0.10(1)$ & 0.73 & $0.80 \pm 0.16$ \\
\hline Y I & 39 & 2.21 & 2.64 & $0.05(4)$ & 0.43 & $1.07 \pm 0.16$ & 2.80 & $-(2)$ & 0.59 & $0.66 \pm 0.16$ \\
\hline Y II & 39 & 2.21 & 2.70 & $0.05(4)$ & 0.49 & $1.06 \pm 0.12$ & 2.91 & $0.13(8)$ & 0.70 & $0.77 \pm 0.12$ \\
\hline Zr I & 40 & 2.58 & 3.23 & $0.10(2)$ & 0.65 & $1.29 \pm 0.11$ & 3.30 & $0.20(2)$ & 0.72 & $0.79 \pm 0.17$ \\
\hline $\mathrm{Zr}$ II & 40 & 2.58 & 3.25 & $0.08(3)$ & 0.67 & $1.31 \pm 0.11$ & 3.70 & $0.10(3)$ & 1.12 & $1.19 \pm 0.12$ \\
\hline $\mathrm{Nb} \mathrm{I}$ & 41 & 1.46 & 2.14 & $0.16(5)$ & 0.68 & $1.32 \pm 0.17$ & 2.18 & $0.10(2)$ & 0.72 & $0.79 \pm 0.17$ \\
\hline Ba II & 56 & 2.18 & 3.3: & - & 1.12 & $1.76 \pm 0.11$ & $3.4:$ & - & 1.22 & $1.29 \pm 0.10$ \\
\hline La II & 57 & 1.10 & 1.89 & $0.13(10)$ & 0.79 & $1.43 \pm 0.12$ & 1.70 & $0.11(7)$ & 0.60 & $0.67 \pm 0.12$ \\
\hline Ce II & 58 & 1.58 & 2.23 & $0.10(8)$ & 0.65 & $1.29 \pm 0.11$ & 2.13 & $0.11(8)$ & 0.55 & $0.62 \pm 0.11$ \\
\hline Pr II & 59 & 0.72 & 1.47 & $0.11(4)$ & 0.75 & $1.39 \pm 0.11$ & 0.98 & $0.05(4)$ & 0.26 & $0.33 \pm 0.10$ \\
\hline Nd II & 60 & 1.42 & 2.13 & $0.10(12)$ & 0.71 & $1.35 \pm 0.10$ & 1.86 & $0.12(12)$ & 0.44 & $0.51 \pm 0.11$ \\
\hline Sm II & 62 & 0.96 & 1.65 & $0.08(2)$ & 0.69 & $1.33 \pm 0.13$ & 1.30 & $0.10(3)$ & 0.34 & $0.41 \pm 0.13$ \\
\hline EuII & 63 & 0.52 & 0.50 & $0.10(4)$ & -0.02 & $0.62 \pm 0.14$ & 0.70 & $0.10(4)$ & 0.18 & $0.25 \pm 0.14$ \\
\hline
\end{tabular}

References. ${ }^{(a)}$ Asplund et al. (2009). 
Table C.1. continued.

\begin{tabular}{|c|c|c|c|c|c|c|c|c|c|c|}
\hline & \multirow[b]{2}{*}{$Z$} & \multirow[b]{2}{*}{$\log _{\odot} \epsilon^{a}$} & \multicolumn{4}{|c|}{ HD 28159} & \multicolumn{4}{|c|}{ HD 31487} \\
\hline & & & $\log \epsilon$ & $\sigma_{s}(\mathrm{~N})$ & {$[\mathrm{X} / \mathrm{H}]$} & {$[\mathrm{X} / \mathrm{Fe}] \pm \sigma_{[X / F e]}$} & $\log \epsilon$ & $\sigma_{s}(\mathrm{~N})$ & {$[\mathrm{X} / \mathrm{H}]$} & {$[\mathrm{X} / \mathrm{Fe}] \pm \sigma_{[X / F e]}$} \\
\hline $\begin{array}{l}\mathrm{C} \\
{ }^{12} \mathrm{C} /{ }^{13} \mathrm{C}\end{array}$ & 6 & 8.43 & $\begin{array}{c}8.30 \\
6\end{array}$ & $-(1)$ & -0.13 & $0.37 \pm 0.13$ & $\begin{array}{c}8.70 \\
16\end{array}$ & $0.03(11)$ & 0.27 & $0.31 \pm 0.08$ \\
\hline $\mathrm{N}$ & 7 & 7.83 & 7.73 & $0.09(45)$ & -0.10 & $0.40 \pm 0.21$ & 8.49 & $0.04(52)$ & 0.66 & $0.70 \pm 0.21$ \\
\hline $\mathrm{O}$ & 8 & 8.69 & 8.60 & $-(2)$ & -0.09 & $0.41 \pm 0.17$ & 8.95 & $-(2)$ & 0.26 & $0.30 \pm 0.17$ \\
\hline $\mathrm{Na} \mathrm{I}$ & 11 & 6.24 & 5.80: & $-(1)$ & -0.44 & $0.06 \pm 0.13$ & 6.63 & $0.07(4)$ & 0.39 & $0.43 \pm 0.12$ \\
\hline Mg I & 12 & 7.60 & 7.60: & $-(1)$ & 0.00 & $0.50 \pm 0.26$ & - & - & - & - \\
\hline $\mathrm{Fe}$ & 26 & 7.50 & 7.00 & $0.15(35)$ & -0.50 & - & 7.46 & $0.19(45)$ & -0.04 & - \\
\hline $\mathrm{Rb} \mathrm{I}$ & 37 & 2.52 & 2.00 & $-(2)$ & -0.52 & $-0.02 \pm 0.13$ & - & - & - & - \\
\hline Sr I & 38 & 2.87 & 2.25 & $0.07(2)$ & -0.62 & $-0.12 \pm 0.11$ & 3.70 & $0.05(2)$ & 0.83 & $0.87 \pm 0.11$ \\
\hline Sr II & 38 & 2.87 & 2.78 & $0.04(2)$ & -0.09 & $0.41 \pm 0.12$ & 3.81 & $0.11(3)$ & 0.94 & $0.98 \pm 0.14$ \\
\hline Y I & 39 & 2.21 & 1.45 & $0.08(3)$ & -0.76 & $-0.26 \pm 0.16$ & 3.03 & $0.09(4)$ & 0.82 & $0.86 \pm 0.16$ \\
\hline Y II & 39 & 2.21 & 1.87 & $0.20(3)$ & -0.34 & $0.16 \pm 0.16$ & 3.40 & $0.13(5)$ & 1.19 & $1.23 \pm 0.13$ \\
\hline Zr I & 40 & 2.58 & 2.38 & $0.10(2)$ & -0.20 & $0.30 \pm 0.12$ & 3.65 & $0.07(2)$ & 1.07 & $1.11 \pm 0.11$ \\
\hline Zr II & 40 & 2.58 & 2.80 & $-(2)$ & 0.22 & $0.72 \pm 0.10$ & 4.27 & $0.10(3)$ & 1.69 & $1.73 \pm 0.12$ \\
\hline $\mathrm{Nb} \mathrm{I}$ & 41 & 1.46 & 1.39 & $0.14(5)$ & -0.07 & $0.43 \pm 0.16$ & 2.57 & $0.06(4)$ & 1.11 & $1.15 \pm 0.16$ \\
\hline Ba II & 56 & 2.18 & 1.28: & - & -0.90 & $-0.40 \pm 0.10$ & 4.00: & - & 1.82 & $1.86 \pm 0.11$ \\
\hline La II & 57 & 1.10 & 1.47 & $0.07(7)$ & 0.37 & $0.87 \pm 0.12$ & 2.59 & $0.13(7)$ & 1.49 & $1.53 \pm 0.12$ \\
\hline Ce II & 58 & 1.58 & 1.54 & $0.19(6)$ & -0.04 & $0.46 \pm 0.13$ & 3.00 & $0.13(10)$ & 1.42 & $1.46 \pm 0.11$ \\
\hline Pr II & 59 & 0.72 & 0.77 & $0.14(4)$ & 0.05 & $0.55 \pm 0.12$ & 2.13 & $0.10(4)$ & 1.41 & $1.45 \pm 0.11$ \\
\hline Nd II & 60 & 1.42 & 1.57 & $0.15(7)$ & 0.15 & $0.65 \pm 0.11$ & 2.84 & $0.12(8)$ & 1.42 & $1.46 \pm 0.11$ \\
\hline Sm II & 62 & 0.96 & 0.85 & $0.20(2)$ & -0.11 & $0.39 \pm 0.18$ & 2.15 & $0.10(2)$ & 1.19 & $1.23 \pm 0.16$ \\
\hline \multirow[t]{3}{*}{ Eu II } & 63 & 0.52 & 0.59 & $0.14(3)$ & 0.07 & $0.57 \pm 0.15$ & 1.08 & $0.08(3)$ & 0.56 & $0.60 \pm 0.14$ \\
\hline & & & \multicolumn{4}{|c|}{ HD 43389} & \multicolumn{4}{|c|}{ HD 46407} \\
\hline & $Z$ & $\log _{\odot} \epsilon^{a}$ & $\log \epsilon$ & $\sigma_{s}(\mathrm{~N})$ & {$[\mathrm{X} / \mathrm{H}]$} & {$[\mathrm{X} / \mathrm{Fe}] \pm \sigma_{[X / F e]}$} & $\log \epsilon$ & $\sigma_{s}(\mathrm{~N})$ & {$[\mathrm{X} / \mathrm{H}]$} & {$[\mathrm{X} / \mathrm{Fe}] \pm \sigma_{[X / F e]}$} \\
\hline $\begin{array}{l}\mathrm{C} \\
{ }^{12} \mathrm{C} /{ }^{13} \mathrm{C}\end{array}$ & 6 & 8.43 & $\begin{array}{c}8.30 \\
10\end{array}$ & $-(1)$ & -0.13 & $0.22 \pm 0.13$ & $\begin{array}{c}8.32 \\
16\end{array}$ & $0.03(10)$ & -0.11 & $0.25 \pm 0.12$ \\
\hline $\mathrm{N}$ & 7 & 7.83 & 8.62 & $0.15(48)$ & 0.79 & $1.14 \pm 0.22$ & 7.95 & $0.10(48)$ & 0.12 & $0.48 \pm 0.20$ \\
\hline $\mathrm{O}$ & 8 & 8.69 & 8.40 & $-(2)$ & -0.29 & $0.06 \pm 0.17$ & 8.40 & $-(1)$ & -0.29 & $0.07 \pm 0.18$ \\
\hline $\mathrm{Na} \mathrm{I}$ & 11 & 6.24 & 5.80 & $0.07(2)$ & -0.44 & $-0.09 \pm 0.13$ & 6.27 & $0.12(3)$ & 0.03 & $0.39 \pm 0.17$ \\
\hline Mg I & 12 & 7.60 & - & - & - & - & 7.30 & $-(2)$ & -0.30 & $0.06 \pm 0.26$ \\
\hline $\mathrm{Fe}$ & 26 & 7.50 & 7.15 & $0.15(30)$ & -0.35 & - & 7.14 & $0.15(39)$ & -0.36 & - \\
\hline $\mathrm{Rb} \mathrm{I}$ & 37 & 2.52 & 1.85 & $-(2)$ & -0.67 & $-0.32 \pm 0.12$ & - & - & - & - \\
\hline Sr I & 38 & 2.87 & 2.80 & $0.10(2)$ & -0.07 & $0.28 \pm 0.14$ & 3.68 & $0.03(2)$ & 0.81 & $1.17 \pm 0.11$ \\
\hline Sr II & 38 & 2.87 & 3.00 & $0.15(3)$ & 0.13 & $0.48 \pm 0.15$ & 3.75 & $0.07(2)$ & 0.88 & $1.24 \pm 0.13$ \\
\hline Y I & 39 & 2.21 & 1.78 & $0.15(3)$ & -0.44 & $-0.09 \pm 0.18$ & 2.85 & $0.17(3)$ & 0.64 & $1.00 \pm 0.19$ \\
\hline Y II & 39 & 2.21 & 2.77 & $0.15(5)$ & 0.56 & $0.91 \pm 0.13$ & 3.00 & $0.15(7)$ & 0.79 & $1.15 \pm 0.13$ \\
\hline Zr I & 40 & 2.58 & 2.55 & $0.10(2)$ & -0.03 & $0.32 \pm 0.12$ & 3.50 & $-(2)$ & 0.92 & $1.28 \pm 0.10$ \\
\hline $\mathrm{Zr}$ II & 40 & 2.58 & - & - & - & - & 3.83 & $0.11(3)$ & 1.25 & $1.61 \pm 0.12$ \\
\hline $\mathrm{Nb} \mathrm{I}$ & 41 & 1.46 & 1.75 & $0.13(9)$ & 0.29 & $0.64 \pm 0.16$ & 2.39 & $0.06(4)$ & 0.93 & $1.29 \pm 0.16$ \\
\hline Ba II & 56 & 2.18 & 3.60: & - & 1.42 & $1.77 \pm 0.11$ & 3.70: & - & 1.52 & $1.88 \pm 0.10$ \\
\hline La II & 57 & 1.10 & 2.28 & $0.13(7)$ & 1.18 & $1.53 \pm 0.12$ & 2.30 & $0.15(6)$ & 1.20 & $1.56 \pm 0.13$ \\
\hline Ce II & 58 & 1.58 & 2.45 & $0.12(7)$ & 0.87 & $1.22 \pm 0.11$ & 2.72 & $0.11(8)$ & 1.14 & $1.50 \pm 0.11$ \\
\hline Pr II & 59 & 0.72 & 1.70 & $0.10(4)$ & 0.98 & $1.33 \pm 0.11$ & 1.76 & $0.13(4)$ & 1.04 & $1.40 \pm 0.10$ \\
\hline Nd II & 60 & 1.42 & 2.50 & $0.14(7)$ & 1.08 & $1.43 \pm 0.11$ & 2.46 & $0.12(14)$ & 1.04 & $1.40 \pm 0.09$ \\
\hline Sm II & 62 & 0.96 & 1.55 & $0.15(2)$ & 0.59 & $0.94 \pm 0.16$ & 1.73 & $0.05(3)$ & 0.77 & $1.13 \pm 0.12$ \\
\hline $\mathrm{Eu}$ II & 63 & 0.52 & 0.93 & $0.03(2)$ & 0.41 & $0.76 \pm 0.13$ & 0.70 & $0.03(4)$ & 0.18 & $0.54 \pm 0.13$ \\
\hline
\end{tabular}


Table C.1. continued.

\begin{tabular}{|c|c|c|c|c|c|c|c|c|c|c|}
\hline & \multirow[b]{2}{*}{$Z$} & \multirow[b]{2}{*}{$\log _{\odot} \epsilon^{a}$} & \multicolumn{4}{|c|}{ HD 50082} & \multicolumn{4}{|c|}{ HD 60197} \\
\hline & & & $\log \epsilon$ & $\sigma_{s}(\mathrm{~N})$ & {$[\mathrm{X} / \mathrm{H}]$} & {$[\mathrm{X} / \mathrm{Fe}] \pm \sigma_{[X / F e]}$} & $\log \epsilon$ & $\sigma_{s}(\mathrm{~N})$ & {$[\mathrm{X} / \mathrm{H}]$} & {$[\mathrm{X} / \mathrm{Fe}] \pm \sigma_{[X / F e]}$} \\
\hline $\begin{array}{l}\mathrm{C} \\
{ }^{12} \mathrm{C} /{ }^{13} \mathrm{C}\end{array}$ & 6 & 8.43 & $\begin{array}{c}8.20 \\
13\end{array}$ & $0.05(11)$ & -0.23 & $0.09 \pm 0.11$ & $\begin{array}{c}8.00 \\
9\end{array}$ & $-(1)$ & -0.43 & $0.17 \pm 0.13$ \\
\hline $\mathrm{N}$ & 7 & 7.83 & 7.70 & $0.08(49)$ & -0.13 & $0.19 \pm 0.21$ & 8.39 & $0.13(47)$ & 0.56 & $1.16 \pm 0.21$ \\
\hline $\mathrm{O}$ & 8 & 8.69 & 8.35 & $-(2)$ & -0.34 & $-0.02 \pm 0.17$ & 8.10 & $-(2)$ & -0.59 & $0.01 \pm 0.17$ \\
\hline $\mathrm{Na} \mathrm{I}$ & 11 & 6.24 & 6.15 & $0.07(2)$ & -0.09 & $0.23 \pm 0.13$ & 6.18 & $0.04(2)$ & -0.06 & $0.54 \pm 0.12$ \\
\hline Mg I & 12 & 7.60 & 7.53 & $0.11(2)$ & -0.07 & $0.25 \pm 0.27$ & - & - & - & - \\
\hline $\mathrm{Fe}$ & 26 & 7.50 & 7.18 & $0.10(36)$ & -0.32 & - & 6.90 & $0.15(30)$ & -0.60 & - \\
\hline $\mathrm{Rb} \mathrm{I}$ & 37 & 2.52 & 2.1: & $-(1)$ & -0.42 & $-0.10 \pm 0.15$ & 2.1 & $-(2)$ & -0.42 & $0.18 \pm 0.13$ \\
\hline Sr I & 38 & 2.87 & 3.40 & $0.03(2)$ & 0.53 & $0.85 \pm 0.11$ & 2.90 & $-(1)$ & 0.03 & $0.63 \pm 0.14$ \\
\hline Sr II & 38 & 2.87 & 3.40 & $0.05(2)$ & 0.53 & $0.85 \pm 0.13$ & 3.13 & $0.08(4)$ & 0.26 & $0.86 \pm 0.13$ \\
\hline Y I & 39 & 2.21 & 2.75 & $0.07(2)$ & 0.54 & $0.86 \pm 0.17$ & 1.88 & $0.15(4)$ & -0.33 & $0.27 \pm 0.18$ \\
\hline Y II & 39 & 2.21 & 2.88 & $0.18(7)$ & 0.67 & $0.99 \pm 0.13$ & 2.55 & $0.12(6)$ & 0.34 & $0.94 \pm 0.12$ \\
\hline Zr I & 40 & 2.58 & 3.30 & $-(2)$ & 0.72 & $1.04 \pm 0.10$ & 2.85 & $-(2)$ & 0.27 & $0.87 \pm 0.10$ \\
\hline Zr II & 40 & 2.58 & 3.60 & $0.05(2)$ & 1.02 & $1.34 \pm 0.11$ & 3.30 & $0.10(2)$ & 0.72 & $1.32 \pm 0.14$ \\
\hline $\mathrm{Nb} \mathrm{I}$ & 41 & 1.46 & 2.28 & $0.12(3)$ & 0.82 & $1.14 \pm 0.17$ & 1.75 & $0.14(9)$ & 0.29 & $0.89 \pm 0.16$ \\
\hline Ba II & 56 & 2.18 & $3.7:$ & - & 1.52 & $1.84 \pm 0.10$ & 3.3: & - & 1.12 & $1.72 \pm 0.10$ \\
\hline La II & 57 & 1.10 & 2.20 & $0.20(5)$ & 1.10 & $1.42 \pm 0.14$ & 1.55 & $0.10(5)$ & 0.45 & $1.05 \pm 0.12$ \\
\hline Ce II & 58 & 1.58 & 2.61 & $0.14(11)$ & 1.03 & $1.35 \pm 0.11$ & 1.86 & $0.14(9)$ & 0.28 & $0.88 \pm 0.10$ \\
\hline Pr II & 59 & 0.72 & 1.73 & $0.16(4)$ & 1.01 & $1.33 \pm 0.13$ & 1.23 & $0.02(4)$ & 0.51 & $1.11 \pm 0.10$ \\
\hline Nd II & 60 & 1.42 & 2.48 & $0.14(14)$ & 1.06 & $1.38 \pm 0.11$ & 1.99 & $0.08(8)$ & 0.57 & $1.17 \pm 0.12$ \\
\hline Sm II & 62 & 0.96 & 1.85 & $0.14(2)$ & 0.89 & $1.21 \pm 0.16$ & 1.33 & $0.03(2)$ & 0.37 & $0.97 \pm 0.12$ \\
\hline \multirow[t]{3}{*}{ Eu II } & 63 & 0.52 & 0.65 & $0.03(4)$ & 0.13 & $0.45 \pm 0.13$ & 0.50 & $0.07(2)$ & -0.02 & $0.58 \pm 0.14$ \\
\hline & & & \multicolumn{4}{|c|}{ HD 88562} & \multicolumn{4}{|c|}{ HD 100503} \\
\hline & $Z$ & $\log _{\odot} \epsilon^{a}$ & $\log \epsilon$ & $\sigma_{s}(\mathrm{~N})$ & {$[\mathrm{X} / \mathrm{H}]$} & {$[\mathrm{X} / \mathrm{Fe}] \pm \sigma_{[X / F e]}$} & $\log \epsilon$ & $\sigma_{s}(\mathrm{~N})$ & {$[\mathrm{X} / \mathrm{H}]$} & {$[\mathrm{X} / \mathrm{Fe}] \pm \sigma_{[X / F e]}$} \\
\hline $\begin{array}{l}\mathrm{C} \\
{ }^{12} \mathrm{C} /{ }^{13} \mathrm{C}\end{array}$ & 6 & 8.43 & $\begin{array}{c}8.00 \\
13\end{array}$ & $-(1)$ & -0.43 & $0.10 \pm 0.13$ & $\begin{array}{c}8.00 \\
10\end{array}$ & $-(1)$ & -0.43 & $0.29 \pm 0.13$ \\
\hline $\mathrm{N}$ & 7 & 7.83 & 7.50 & $0.13(45)$ & -0.33 & $0.20 \pm 0.22$ & 8.32 & $0.13(48)$ & 0.49 & $1.21 \pm 0.21$ \\
\hline $\mathrm{O}$ & 8 & 8.69 & 8.05 & $-(2)$ & -0.64 & $-0.11 \pm 0.18$ & 8.03 & $-(2)$ & -0.66 & $0.06 \pm 0.18$ \\
\hline $\mathrm{Na} \mathrm{I}$ & 11 & 6.24 & 5.80 & $0.07(2)$ & -0.44 & $0.09 \pm 0.13$ & 6.10 & $0.14(2)$ & -0.14 & $0.58 \pm 0.15$ \\
\hline Mg I & 12 & 7.60 & 7.00: & $-(1)$ & -0.60 & $-0.07 \pm 0.26$ & 7.20: & $-(1)$ & -0.40 & $0.32 \pm 0.27$ \\
\hline $\mathrm{Fe}$ & 26 & 7.50 & 6.97 & $0.15(30)$ & -0.53 & - & 6.78 & $0.15(35)$ & -0.72 & - \\
\hline $\mathrm{Rb} \mathrm{I}$ & 37 & 2.52 & 1.85 & $0.03(2)$ & -0.67 & $-0.14 \pm 0.13$ & 2.00 & $-(1)$ & -0.52 & $0.20 \pm 0.13$ \\
\hline Sr I & 38 & 2.87 & 2.75 & $0.08(3)$ & -0.12 & $0.41 \pm 0.11$ & 2.95 & $-(1)$ & 0.08 & $0.80 \pm 0.14$ \\
\hline Sr II & 38 & 2.87 & 2.90 & $0.05(3)$ & 0.05 & $0.56 \pm 0.12$ & 3.10 & $0.14(2)$ & 0.23 & $0.95 \pm 0.14$ \\
\hline Y I & 39 & 2.21 & 1.75 & $0.14(4)$ & -0.46 & $0.07 \pm 0.17$ & 2.16 & $0.12(4)$ & -0.05 & $0.67 \pm 0.16$ \\
\hline Y II & 39 & 2.21 & 2.61 & $0.10(7)$ & 0.40 & $0.93 \pm 0.12$ & 2.80 & $0.10(3)$ & 0.59 & $1.31 \pm 0.13$ \\
\hline Zr I & 40 & 2.58 & 2.48 & $0.10(2)$ & -0.10 & $0.43 \pm 0.12$ & 2.86 & $0.10(2)$ & 0.18 & $1.00 \pm 0.12$ \\
\hline $\mathrm{Zr}$ II & 40 & 2.58 & 3.42 & $0.04(3)$ & 0.84 & $1.37 \pm 0.11$ & 3.3: & $-(1)$ & 0.72 & $1.44 \pm 0.14$ \\
\hline $\mathrm{Nb} \mathrm{I}$ & 41 & 1.46 & 1.46 & $0.10(9)$ & 0.00 & $0.53 \pm 0.16$ & 2.07 & $0.14(7)$ & 0.61 & $1.33 \pm 0.16$ \\
\hline Ba II & 56 & 2.18 & 3.3: & - & 1.12 & $1.65 \pm 0.10$ & 2.85: & - & 0.67 & $1.39 \pm 0.10$ \\
\hline La II & 57 & 1.10 & 1.72 & $0.10(9)$ & 0.62 & $1.15 \pm 0.12$ & 2.17 & $0.09(7)$ & 1.07 & $1.79 \pm 0.12$ \\
\hline Ce II & 58 & 1.58 & 2.07 & $0.10(10)$ & 0.49 & $1.02 \pm 0.11$ & 2.49 & $0.12(5)$ & 0.91 & $1.63 \pm 0.12$ \\
\hline Pr II & 59 & 0.72 & 1.24 & $0.08(4)$ & 0.52 & $1.05 \pm 0.11$ & 1.78 & $0.20(4)$ & 1.06 & $1.78 \pm 0.14$ \\
\hline Nd II & 60 & 1.42 & 1.90 & $0.14(11)$ & 0.48 & $1.01 \pm 0.11$ & 2.58 & $0.04(6)$ & 1.16 & $1.88 \pm 0.10$ \\
\hline Sm II & 62 & 0.96 & 1.25 & $0.08(2)$ & 0.29 & $0.82 \pm 0.13$ & 1.91 & $0.13(2)$ & 0.95 & $1.67 \pm 0.15$ \\
\hline Eu II & 63 & 0.52 & 0.70 & $0.10(2)$ & 0.18 & $0.71 \pm 0.15$ & 1.02 & $0.03(2)$ & 0.50 & $1.22 \pm 0.13$ \\
\hline
\end{tabular}


Table C.1. continued.

\begin{tabular}{|c|c|c|c|c|c|c|c|c|c|c|}
\hline & \multirow[b]{2}{*}{$Z$} & \multirow[b]{2}{*}{$\log _{\odot} \epsilon^{a}$} & \multicolumn{4}{|c|}{ HD 116869} & \multicolumn{4}{|c|}{ HD 120620} \\
\hline & & & $\log \epsilon$ & $\sigma_{s}(\mathrm{~N})$ & {$[\mathrm{X} / \mathrm{H}]$} & {$[\mathrm{X} / \mathrm{Fe}] \pm \sigma_{[X / F e]}$} & $\log \epsilon$ & $\sigma_{s}(\mathrm{~N})$ & {$[\mathrm{X} / \mathrm{H}]$} & {$[\mathrm{X} / \mathrm{Fe}] \pm \sigma_{[X / F e]}$} \\
\hline $\begin{array}{l}\mathrm{C} \\
{ }^{12} \mathrm{C} /{ }^{13} \mathrm{C}\end{array}$ & 6 & 8.43 & $\begin{array}{c}8.30 \\
9\end{array}$ & $0.03(10)$ & -0.13 & $0.31 \pm 0.12$ & $\begin{array}{c}8.60 \\
90\end{array}$ & $0.03(7)$ & 0.17 & $0.47 \pm 0.12$ \\
\hline $\mathrm{N}$ & 7 & 7.83 & 7.70 & $0.05(49)$ & -0.13 & $0.31 \pm 0.21$ & 8.00 & $0.05(52)$ & 0.17 & $0.47 \pm 0.20$ \\
\hline $\mathrm{O}$ & 8 & 8.69 & 8.60 & $-(2)$ & -0.09 & $0.35 \pm 0.16$ & 8.90 & $-(1)$ & 0.21 & $0.51 \pm 0.17$ \\
\hline $\mathrm{Na} \mathrm{I}$ & 11 & 6.24 & 6.05 & $0.07(2)$ & -0.19 & $0.25 \pm 0.13$ & 6.22 & $0.03(3)$ & -0.02 & $0.28 \pm 0.15$ \\
\hline Mg I & 12 & 7.60 & 7.60 & $-(2)$ & 0.00 & $0.44 \pm 0.26$ & 7.60: & - & 0.00 & $0.30 \pm 0.24$ \\
\hline $\mathrm{Fe}$ & 26 & 7.50 & 7.06 & $0.15(40)$ & -0.44 & - & 7.2 & $0.16(42)$ & -0.30 & - \\
\hline $\mathrm{Rb} \mathrm{I}$ & 37 & 2.52 & - & - & - & - & - & - & - & - \\
\hline Sr I & 38 & 2.87 & 3.30 & $-(1)$ & 0.43 & $0.87 \pm 0.10$ & 3.75 & $-(2)$ & 0.88 & $1.18 \pm 0.10$ \\
\hline Sr II & 38 & 2.87 & 3.30 & $-(2)$ & 0.43 & $0.87 \pm 0.12$ & 4.00 & $0.10(2)$ & 1.13 & $1.43 \pm 0.16$ \\
\hline Y I & 39 & 2.21 & 2.30 & $-(1)$ & 0.09 & $0.53 \pm 0.19$ & 3.03 & $0.12(4)$ & 0.82 & $1.12 \pm 0.17$ \\
\hline Y II & 39 & 2.21 & 2.48 & $0.12(6)$ & 0.27 & $0.71 \pm 0.12$ & 3.20 & $0.16(7)$ & 0.99 & $1.29 \pm 0.13$ \\
\hline Zr I & 40 & 2.58 & 3.15 & $0.20(2)$ & 0.57 & $1.01 \pm 0.17$ & 3.55 & $-(1)$ & 0.97 & $1.27 \pm 0.14$ \\
\hline Zr II & 40 & 2.58 & 3.18 & $0.10(3)$ & 0.60 & $1.04 \pm 0.12$ & 4.04 & $0.07(3)$ & 1.46 & $1.76 \pm 0.11$ \\
\hline $\mathrm{Nb} \mathrm{I}$ & 41 & 1.46 & 2.00 & $0.15(4)$ & 0.54 & $0.98 \pm 0.21$ & 2.51 & $0.13(8)$ & 1.05 & $1.35 \pm 0.16$ \\
\hline Ba II & 56 & 2.18 & $3.15:$ & - & 0.97 & $1.41 \pm 0.10$ & $3.9:$ & - & 1.72 & $2.02 \pm 0.10$ \\
\hline La II & 57 & 1.10 & 1.82 & $0.10(9)$ & 0.72 & $1.16 \pm 0.12$ & 2.40 & $0.16(6)$ & 1.30 & $1.60 \pm 0.13$ \\
\hline Ce II & 58 & 1.58 & 2.21 & $0.09(10)$ & 0.63 & $1.07 \pm 0.11$ & 2.80 & $0.15(8)$ & 1.22 & $1.52 \pm 0.11$ \\
\hline Pr II & 59 & 0.72 & 1.20 & $0.08(4)$ & 0.48 & $0.92 \pm 0.11$ & 1.93 & $0.06(4)$ & 1.21 & $1.51 \pm 0.10$ \\
\hline Nd II & 60 & 1.42 & 2.10 & $0.09(12)$ & 0.68 & $1.12 \pm 0.10$ & 2.60 & $0.06(14)$ & 1.18 & $1.48 \pm 0.10$ \\
\hline Sm II & 62 & 0.96 & 1.58 & $0.13(4)$ & 0.62 & $1.06 \pm 0.14$ & 1.85 & $0.14(4)$ & 0.89 & $1.19 \pm 0.14$ \\
\hline \multirow[t]{3}{*}{ Eu II } & 63 & 0.52 & 0.65 & $0.08(2)$ & 0.13 & $0.57 \pm 0.14$ & 0.97 & $0.03(4)$ & 0.45 & $0.75 \pm 0.13$ \\
\hline & & & \multicolumn{4}{|c|}{ HD 121447} & \multicolumn{4}{|c|}{ HD 123949} \\
\hline & $Z$ & $\log _{\odot} \epsilon^{a}$ & $\log \epsilon$ & $\sigma_{s}(\mathrm{~N})$ & {$[\mathrm{X} / \mathrm{H}]$} & {$[\mathrm{X} / \mathrm{Fe}] \pm \sigma_{[X / F e]}$} & $\log \epsilon$ & $\sigma_{s}(\mathrm{~N})$ & {$[\mathrm{X} / \mathrm{H}]$} & {$[\mathrm{X} / \mathrm{Fe}] \pm \sigma_{[X / F e]}$} \\
\hline $\begin{array}{l}\mathrm{C} \\
{ }^{12} \mathrm{C} /{ }^{13} \mathrm{C}\end{array}$ & 6 & 8.43 & $\begin{array}{c}8.07 \\
7\end{array}$ & $-(1)$ & -0.36 & $0.54 \pm 0.13$ & $\begin{array}{c}8.40 \\
19\end{array}$ & $0.05(10)$ & -0.03 & $0.28 \pm 0.12$ \\
\hline $\mathrm{N}$ & 7 & 7.83 & 7.80 & $0.13(45)$ & -0.03 & $0.87 \pm 0.21$ & 7.70 & $0.06(50)$ & -0.13 & $0.18 \pm 0.21$ \\
\hline $\mathrm{O}$ & 8 & 8.69 & 8.09 & $-(2)$ & -0.60 & $0.30 \pm 0.17$ & 8.45 & $-(1)$ & -0.24 & $0.07 \pm 0.18$ \\
\hline $\mathrm{Na} \mathrm{I}$ & 11 & 6.24 & 6.23 & $0.03(2)$ & -0.01 & $0.89 \pm 0.07$ & 6.27 & $0.07(3)$ & 0.03 & $0.34 \pm 0.12$ \\
\hline Mg I & 12 & 7.60 & - & - & - & - & 7.60 & $-(2)$ & 0.00 & $0.31 \pm 0.26$ \\
\hline $\mathrm{Fe}$ & 26 & 7.50 & 6.60 & $0.15(28)$ & -0.90 & - & 7.19 & $0.18(41)$ & -0.31 & - \\
\hline $\mathrm{Rb} \mathrm{I}$ & 37 & 2.52 & 2.25 & $0.05(2)$ & -0.27 & $0.63 \pm 0.13$ & - & - & - & - \\
\hline $\mathrm{Sr} \mathrm{I}$ & 38 & 2.87 & 3.18 & $0.03(2)$ & 0.31 & $1.21 \pm 0.11$ & 3.44 & $0.11(3)$ & 0.57 & $0.88 \pm 0.12$ \\
\hline Sr II & 38 & 2.87 & 3.5: & $-(1)$ & 0.63 & $1.53 \pm 0.13$ & 3.46 & $0.08(3)$ & 0.59 & $0.90 \pm 0.13$ \\
\hline Y I & 39 & 2.21 & 2.38 & $0.09(4)$ & 0.17 & $1.07 \pm 0.16$ & 2.65 & $0.15(4)$ & 0.44 & $0.75 \pm 0.18$ \\
\hline Y II & 39 & 2.21 & 2.66 & $0.11(5)$ & 0.45 & $1.35 \pm 0.12$ & 2.81 & $0.17(5)$ & 0.60 & $0.91 \pm 0.14$ \\
\hline Zr I & 40 & 2.58 & 3.25 & $0.14(2)$ & 0.67 & $1.57 \pm 0.14$ & 3.15 & $0.14(2)$ & 0.57 & $0.88 \pm 0.14$ \\
\hline $\mathrm{Zr}$ II & 40 & 2.58 & - & - & - & - & 3.73 & $0.10(3)$ & 1.15 & $1.46 \pm 0.12$ \\
\hline $\mathrm{Nb} \mathrm{I}$ & 41 & 1.46 & 2.48 & $0.10(7)$ & 1.02 & $1.92 \pm 0.16$ & 2.11 & $0.12(9)$ & 0.65 & $0.96 \pm 0.16$ \\
\hline Ba II & 56 & 2.18 & $3.15:$ & - & 0.97 & $1.87 \pm 0.11$ & $3.8:$ & - & 1.62 & $1.93 \pm 0.10$ \\
\hline La II & 57 & 1.10 & 2.59 & $0.15(5)$ & 1.49 & $2.39 \pm 0.13$ & 2.00 & $0.14(7)$ & 0.90 & $1.21 \pm 0.13$ \\
\hline Ce II & 58 & 1.58 & 2.90 & $0.17(3)$ & 1.32 & $2.22 \pm 0.14$ & 2.55 & $0.14(8)$ & 0.97 & $1.28 \pm 0.11$ \\
\hline Pr II & 59 & 0.72 & 2.18 & $0.08(4)$ & 1.46 & $2.36 \pm 0.11$ & 1.77 & $0.13(4)$ & 1.05 & $1.36 \pm 0.12$ \\
\hline Nd II & 60 & 1.42 & 2.55 & $0.12(6)$ & 1.13 & $2.03 \pm 0.11$ & 2.45 & $0.13(12)$ & 1.03 & $1.34 \pm 0.11$ \\
\hline Sm II & 62 & 0.96 & 1.85 & $0.17(2)$ & 0.89 & $1.79 \pm 0.17$ & 1.70 & $0.10(3)$ & 0.74 & $1.05 \pm 0.13$ \\
\hline $\mathrm{Eu}$ II & 63 & 0.52 & 0.75 & $0.03(2)$ & 0.23 & $1.13 \pm 0.13$ & 0.70 & $0.10(3)$ & 0.18 & $0.49 \pm 0.14$ \\
\hline
\end{tabular}


Table C.1. continued.

\begin{tabular}{|c|c|c|c|c|c|c|c|c|c|c|}
\hline & \multirow[b]{2}{*}{$Z$} & \multirow[b]{2}{*}{$\log _{\odot} \epsilon^{a}$} & \multicolumn{4}{|c|}{ HD 178717} & \multicolumn{4}{|c|}{ HD 199939} \\
\hline & & & $\log \epsilon$ & $\sigma_{s}(\mathrm{~N})$ & {$[\mathrm{X} / \mathrm{H}]$} & {$[\mathrm{X} / \mathrm{Fe}] \pm \sigma_{[X / F e]}$} & $\log \epsilon$ & $\sigma_{s}(\mathrm{~N})$ & {$[\mathrm{X} / \mathrm{H}]$} & {$[\mathrm{X} / \mathrm{Fe}] \pm \sigma_{[X / F e]}$} \\
\hline $\begin{array}{l}\mathrm{C} \\
{ }^{12} \mathrm{C} /{ }^{13} \mathrm{C}\end{array}$ & 6 & 8.43 & $\begin{array}{c}8.00 \\
14\end{array}$ & $-(1)$ & -0.43 & $0.09 \pm 0.13$ & $\begin{array}{c}8.50 \\
19\end{array}$ & $0.06(7)$ & 0.07 & $0.29 \pm 0.12$ \\
\hline $\mathrm{N}$ & 7 & 7.83 & 7.39 & $0.11(45)$ & -0.44 & $0.08 \pm 0.21$ & 8.30 & $0.08(49)$ & 0.47 & $0.69 \pm 0.20$ \\
\hline $\mathrm{O}$ & 8 & 8.69 & 8.003 & $-(1)$ & -0.69 & $-0.17 \pm 0.18$ & 8.75 & $-(1)$ & 0.06 & $0.28 \pm 0.18$ \\
\hline $\mathrm{Na} \mathrm{I}$ & 11 & 6.24 & 5.70 & $0.07(2)$ & -0.54 & $-0.02 \pm 0.13$ & 6.38 & $0.10(2)$ & 0.14 & $0.36 \pm 0.14$ \\
\hline $\mathrm{Mg} \mathrm{I}$ & 12 & 7.60 & 7.40: & $-(1)$ & -0.20 & $0.32 \pm 0.26$ & 7.43 & $0.06(3)$ & -0.17 & $0.05 \pm 0.24$ \\
\hline $\mathrm{Fe}$ & 26 & 7.50 & 6.98 & $0.15(30)$ & -0.52 & - & 7.28 & $0.11(48)$ & -0.22 & - \\
\hline $\mathrm{Rb} \mathrm{I}$ & 37 & 2.52 & 2.00 & $-(1)$ & -0.52 & $0.00 \pm 0.13$ & - & - & - & - \\
\hline Sr I & 38 & 2.87 & 2.95 & $0.07(2)$ & 0.08 & $0.60 \pm 0.11$ & 3.70 & $-(2)$ & 0.83 & $1.05 \pm 0.10$ \\
\hline Sr II & 38 & 2.87 & 3.10 & $0.08(3)$ & 0.23 & $0.75 \pm 0.13$ & 3.90 & $0.15(2)$ & 1.03 & $1.25 \pm 0.16$ \\
\hline Y I & 39 & 2.21 & 1.73 & $0.17(4)$ & -0.48 & $0.04 \pm 0.18$ & 2.99 & $0.11(4)$ & 0.78 & $1.00 \pm 0.17$ \\
\hline Y II & 39 & 2.21 & 2.48 & $0.10(6)$ & 0.27 & $0.79 \pm 0.12$ & 3.37 & $0.18(7)$ & 1.16 & $1.38 \pm 0.13$ \\
\hline Zr I & 40 & 2.58 & 2.50 & $0.20(2)$ & -0.08 & $0.44 \pm 0.17$ & 3.55 & $0.07(2)$ & 0.97 & $1.19 \pm 0.13$ \\
\hline $\mathrm{Zr}$ II & 40 & 2.58 & 3.35 & $0.07(2)$ & 0.77 & $1.29 \pm 0.11$ & 4.10 & $-(2)$ & 1.52 & $1.74 \pm 0.10$ \\
\hline $\mathrm{Nb} \mathrm{I}$ & 41 & 1.46 & 1.62 & $0.12(9)$ & 0.16 & $0.68 \pm 0.16$ & 2.57 & $0.09(9)$ & 1.11 & $1.33 \pm 0.16$ \\
\hline Ba II & 56 & 2.18 & 2.70: & - & 0.52 & $1.04 \pm 0.10$ & $4.2:$ & - & 2.02 & $2.24 \pm 0.10$ \\
\hline La II & 57 & 1.10 & 1.43 & $0.04(7)$ & 0.33 & $0.85 \pm 0.11$ & 2.60 & $0.20(4)$ & 1.50 & $1.72 \pm 0.15$ \\
\hline Ce II & 58 & 1.58 & 1.80 & $0.11(8)$ & 0.22 & $0.74 \pm 0.11$ & 3.03 & $0.11(6)$ & 1.45 & $1.67 \pm 0.11$ \\
\hline Pr II & 59 & 0.72 & 1.08 & $0.07(4)$ & 0.36 & $0.88 \pm 0.10$ & 2.30 & $0.12(4)$ & 1.58 & $1.80 \pm 0.12$ \\
\hline Nd II & 60 & 1.42 & 1.82 & $0.14(7)$ & 0.40 & $0.92 \pm 0.11$ & 2.92 & $0.12(14)$ & 1.50 & $1.72 \pm 0.10$ \\
\hline Sm II & 62 & 0.96 & 1.08 & $0.10(2)$ & 0.12 & $0.64 \pm 0.14$ & 2.30 & $0.03(2)$ & 1.34 & $1.56 \pm 0.12$ \\
\hline Eu II & 63 & 0.52 & 0.55 & $0.10(2)$ & 0.03 & $0.55 \pm 0.15$ & 1.00 & $0.03(2)$ & 0.48 & $0.70 \pm 0.13$ \\
\hline
\end{tabular}

